Supporting Information

\title{
Discovery of Polycyclic Macrolide Shuangdaolides by Heterologous Expression of a Cryptic trans-AT PKS Gene Cluster
}

Yang Liu, ${ }^{\dagger, \perp}$ Haibo Zhou,${ }^{\dagger, \perp}$ Qiyao Shen,${ }^{\dagger}$ Guangzhi Dai, ${ }^{\dagger}$ Fu Yan, ${ }^{\dagger}$ Xiaoju Li,,${ }^{\dagger}$ Xiangmei Ren, ${ }^{\ddagger}$ Qin Sun,${ }^{\S}$ Ya-Jie Tang, ${ }^{\dagger}$ Youming Zhang, ${ }^{\dagger}$ and Xiaoying Bian ${ }^{\dagger}, *$

${ }^{\dagger}$ Helmholtz International Lab for Anti-infectives, Shandong University-Helmholtz

Institute of Biotechnology, State Key Laboratory of Microbial Technology, Shandong University, Qingdao, Shandong 266237, China

$\$$ Core Facilities for Life and Environmental Sciences, State Key Laboratory of Microbial Technology, Shandong University, Qingdao, Shandong 266237, China

${ }^{\S}$ Shenyang Sinochem Agrochemicals R\&D Co. Ltd, Shenyang, Liaoning 110021, China 


\section{Content}

Content

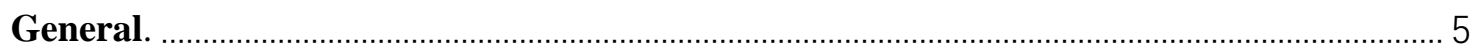

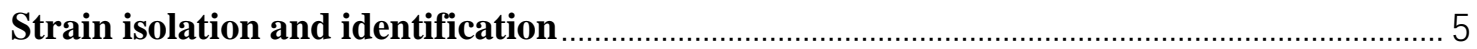

Genome sequencing and bioinformatics analysis.

Direct cloning of $s d l$ gene cluster and construction of expression vector ................................ 5

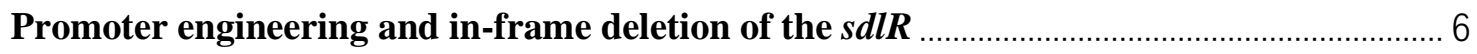

Heterologous expression, fermentation, and isolation of shuangdaolide in S. albus $\mathbf{J 1 0 7 4} 7$

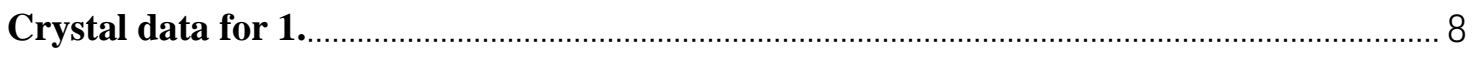

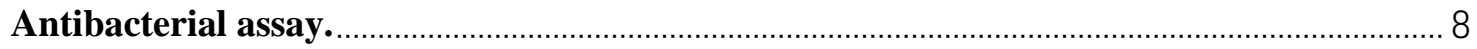

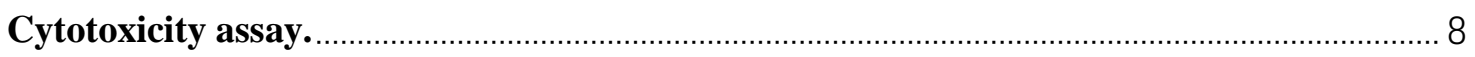

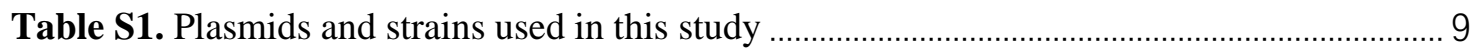

Table S2. Primers used in this work .............................................................................................. 10

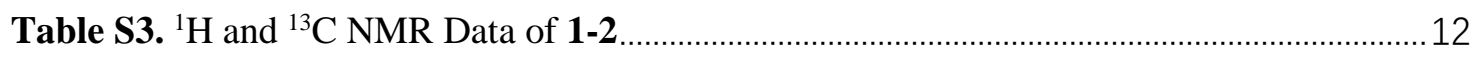

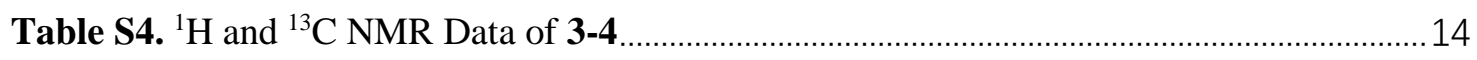

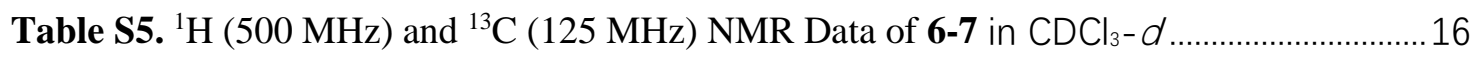

Table S6. Putative functions of the genes in the $s d l$ gene cluster ................................................ 17

Table S7. Predicted KS substrates in shuangdaolide biosynthesis by the TransATor ${ }^{13}$. (Black: correct prediction; red: incorrect prediction.)......................................................................... 19

Table S8. Predicted stereospecificities of KR domains in the shuangdaolide biosynthesis. ..... 20

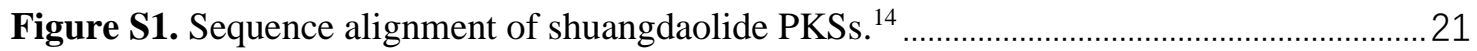

Figure S2. Restriction analysis of pBAC1610 (A) and pBAC1611 (B). ................................... 22

Figure S3. the promoter optimization of $s d l$ gene cluster.........................................................22

Figure S4. In-frame deletion and trans-complement of the $s d l R$ gene.......................................23

Figure S5. Key COSY, HMBC, and NOESY correlations of 2-4 and 6-7................................. 24

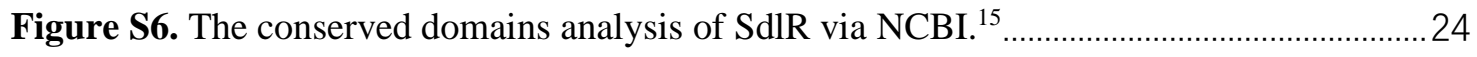

Figure S7. Presumed post-modification of shuangdaolides..................................................... 25

Figure S8. The X-ray structure of $\mathbf{1}$ with the ellipsoid contour at $50 \%$ probability levels. .......25

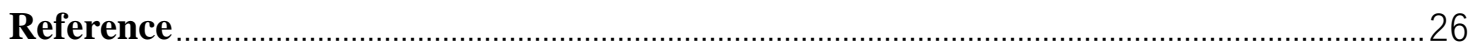

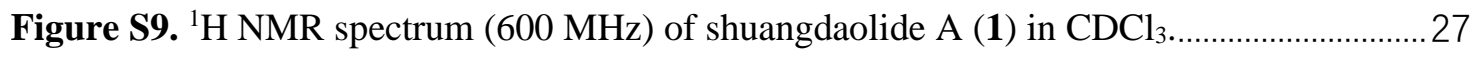

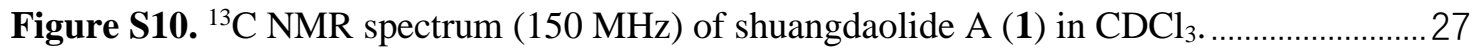


Figure S11. DEPT spectrum (150 MHz) of shuangdaolide A (1) in $\mathrm{CDCl}_{3}$. 28

Figure S12. HSQC spectrum (150 MHz/600 MHz) of shuangdaolide A (1) in $\mathrm{CDCl}_{3 \ldots \ldots \ldots \ldots . . .28}$

Figure S13. ${ }^{1} \mathrm{H}-{ }^{1} \mathrm{H}$ COSY spectrum $(600 \mathrm{MHz})$ of shuangdaolide $\mathrm{A}(\mathbf{1})$ in $\mathrm{CDCl}_{3} \ldots \ldots \ldots \ldots \ldots . . . . . . .29$

Figure S14. HMBC spectrum (150 MHz/600 MHz) of shuangdaolide A (1) in $\mathrm{CDCl}_{3}$..........29

Figure S15. NOESY spectrum $(600 \mathrm{MHz})$ of shuangdaolide A (1) in $\mathrm{CDCl}_{3} \ldots \ldots \ldots \ldots \ldots \ldots \ldots \ldots . . . . . . . . . . . . . .30$

Figure S16. ${ }^{1} \mathrm{H}$ NMR spectrum $(500 \mathrm{MHz})$ of shuangdaolide B (2) in DMSO- $d_{6} \ldots \ldots \ldots \ldots \ldots \ldots . . . . . . . . . .30$

Figure S17. ${ }^{13} \mathrm{C}$ NMR spectrum $(125 \mathrm{MHz})$ of shuangdaolide B (2) in DMSO- $d_{6} \ldots \ldots \ldots \ldots \ldots \ldots . . . . . . . .31$

Figure S18. DEPT spectrum (125 MHz) of shuangdaolide B (2) in DMSO- $d_{6} \ldots \ldots \ldots \ldots \ldots \ldots \ldots \ldots . . . . . . . . . . . . .31$

Figure S19. HSQC spectrum (125 MHz/500 MHz) of shuangdaolide B (2) in DMSO- $d_{6} \ldots \ldots . . .32$

Figure S20. ${ }^{1} \mathrm{H}-{ }^{1} \mathrm{H}$ COSY spectrum $(500 \mathrm{MHz})$ of shuangdaolide B (2) in DMSO- $d_{6} \ldots \ldots \ldots \ldots . . . . .32$

Figure S21. HMBC spectrum (125 MHz/500 MHz) of shuangdaolide B (2) in DMSO- $d_{6} \ldots . . . .33$

Figure S22. NOESY spectrum $(500 \mathrm{MHz})$ of shuangdaolide B (2) in DMSO- $d_{6} \ldots \ldots \ldots \ldots \ldots \ldots \ldots . . . . . . . . .33$

Figure S23. ${ }^{1} \mathrm{H}$ NMR spectrum $(600 \mathrm{MHz})$ of shuangdaolide $\mathrm{C}(\mathbf{3})$ in $\mathrm{CDCl}_{3}$. ........................ 34

Figure S24. ${ }^{13} \mathrm{C}$ NMR spectrum $(150 \mathrm{MHz})$ of shuangdaolide $\mathrm{C}(3)$ in $\mathrm{CDCl}_{3}$...................... 34

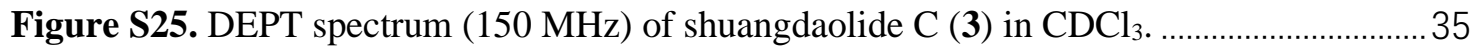

Figure S26. HSQC spectrum (150 MHz/600 MHz) of shuangdaolide C (3) in $\mathrm{CDCl}_{3} \ldots \ldots \ldots \ldots . . . .35$

Figure S27. ${ }^{1} \mathrm{H}-{ }^{1} \mathrm{H}$ COSY spectrum $(600 \mathrm{MHz})$ of shuangdaolide $\mathrm{C}(3)$ in $\mathrm{CDCl}_{3} \ldots \ldots \ldots \ldots \ldots . . . . .36$

Figure S28. HMBC spectrum (150 MHz/600 MHz) of shuangdaolide C (3) in $\mathrm{CDCl}_{3 \ldots \ldots \ldots . . . .36}$

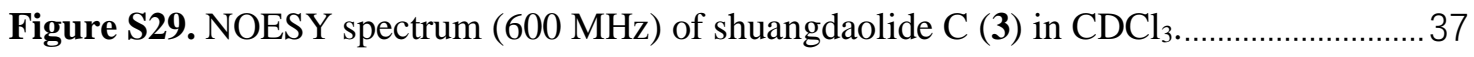

Figure S30. ${ }^{1} \mathrm{H}$ NMR spectrum $(600 \mathrm{MHz})$ of shuangdaolide D (4) in DMSO- $d_{6} \ldots \ldots \ldots \ldots \ldots \ldots \ldots . . . . . . . . .37$

Figure S31. ${ }^{13} \mathrm{C}$ NMR spectrum $(150 \mathrm{MHz})$ of shuangdaolide D (4) in DMSO- $d_{6} \ldots \ldots \ldots \ldots \ldots \ldots . . . . . . . .38$

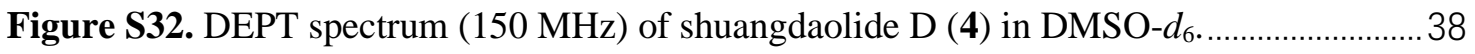

Figure S33. HSQC spectrum (150 MHz/600 MHz) of shuangdaolide D (4) in DMSO- $d_{6} \ldots . . . .39$

Figure S34. ${ }^{1} \mathrm{H}-{ }^{1} \mathrm{H}$ COSY spectrum (600 MHz) of shuangdaolide D (4) in DMSO-d $d_{6} \ldots \ldots \ldots \ldots . . . . .39$

Figure S35. HMBC spectrum (150 MHz/600 MHz) of shuangdaolide D (4) in DMSO- $d_{6} \ldots . . .40$

Figure S36. NOESY spectrum $(600 \mathrm{MHz})$ of shuangdaolide D (4) in DMSO- $d_{6} \ldots \ldots \ldots \ldots \ldots \ldots . . . . . . . . . . .40$

Figure S37. ${ }^{1} \mathrm{H}$ NMR spectrum $(500 \mathrm{MHz})$ of shuangdaolide $\mathrm{F}(\mathbf{6})$ in $\mathrm{CDCl}_{3} \ldots \ldots \ldots \ldots \ldots \ldots \ldots . . . . . . . . . . . .41$

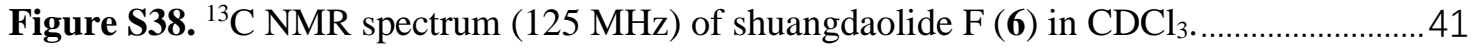

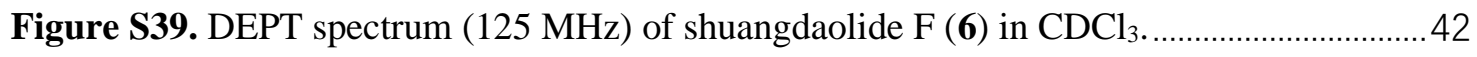

Figure S40. HSQC spectrum (125 MHz/500 MHz) of shuangdaolide $\mathrm{F}(\mathbf{6})$ in $\mathrm{CDCl}_{3}$............ 42

Figure S41. ${ }^{1} \mathrm{H}-{ }^{1} \mathrm{H}$ COSY spectrum $(500 \mathrm{MHz})$ of shuangdaolide $\mathrm{F}(6)$ in $\mathrm{CDCl}_{3} \ldots \ldots \ldots \ldots \ldots \ldots . .43$ 
Figure S42. HMBC spectrum (125 MHz/500 MHz) of shuangdaolide $\mathrm{F}(6)$ in $\mathrm{CDCl}_{3}$.

Figure S43. NOESY spectrum (500 MHz) of shuangdaolide $\mathrm{F}(\mathbf{6})$ in $\mathrm{CDCl}_{3} \ldots \ldots \ldots \ldots \ldots \ldots \ldots \ldots . . . . . . . . . . . .44$

Figure S44. ${ }^{1} \mathrm{H}$ NMR spectrum (500 MHz) of shuangdaolide $\mathrm{G}(7)$ in $\mathrm{CDCl}_{3}$....................... 44

Figure S45. ${ }^{13} \mathrm{C}$ NMR spectrum $(125 \mathrm{MHz})$ of shuangdaolide $\mathrm{G}(7)$ in $\mathrm{CDCl}_{3} \ldots \ldots \ldots \ldots \ldots \ldots \ldots . . . . . . . . . . .45$

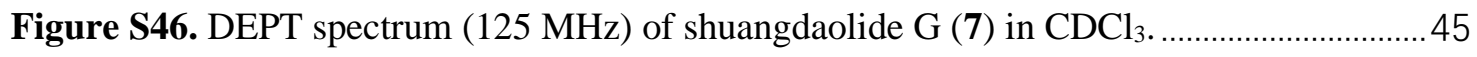

Figure S47. HSQC spectrum (125 MHz/500 MHz) of shuangdaolide $\mathrm{G}(7)$ in $\mathrm{CDCl}_{3} \ldots \ldots \ldots \ldots . . . .46$

Figure S48. ${ }^{1} \mathrm{H}-{ }^{1} \mathrm{H}$ COSY spectrum $(500 \mathrm{MHz})$ of shuangdaolide $\mathrm{G}(7)$ in $\mathrm{CDCl}_{3} \ldots \ldots \ldots \ldots \ldots . . .46$

Figure S49. HMBC spectrum (125 MHz/500 MHz) of shuangdaolide $\mathrm{G}(7)$ in $\mathrm{CDCl}_{3} \ldots \ldots \ldots . . .47$

Figure S50. NOESY spectrum $(500 \mathrm{MHz})$ of shuangdaolide $\mathrm{G}(7)$ in $\mathrm{CDCl}_{3}$........................ 47 


\section{Materials and Methods}

\section{General.}

All the analytical LC-MS data were collected on a Bruker amazon SL Ion Trap mass spectrometer coupled with a Thermo Fisher UltiMate 3000 HPLC using a reversed-phase C18 column $(2.1 \times 100 \mathrm{~mm}$, $2.2 \mu \mathrm{m}$, Thermo). The high-resolution electrospray ionization mass spectrometry (HRESIMS) data were obtained on a Bruker Impact HD microTOF Q III mass spectrometer. Semipreparative RP-HPLC was performed on an Agilent 1260 HPLC and a Shimadzu Essentia LC-16 HPLC equipped with C1 8 column (YMC-pack ODS-A, $10 \times 250 \mathrm{~mm}, 5 \mu \mathrm{m}$ ). 1D and 2D NMR spectra were recorded on an Agilent ProPulse $500 \mathrm{MHz}$ and a Bruker Avance NEO 600MHz. Optical rotation value was measured on a JASCO P-1020 digital polarimeter. IR spectra were acquired on a Nicolet NEXUS 470 spectrophotometer in $\mathrm{KBr}$ disks. The crystal data were collected from a Rigaku AFC10K Saturn 944+ CCD-based X-ray diffractometer.

\section{Strain isolation and identification}

Streptomyces sp. B59 was isolated from the marine sediment collected at the coast near Shuangdaowan Bay, Weihai, China, in 2015. The genome sequence of the strain holds 6 copies of 16S rRNA genes, three genes (MZ148637, MZ148640, and MZ148643) of which differentiate each other with one base pair. Strain B59 was most closely related to Streptomyces sp. H2AK (100\% identity, accession \# MN626600)

\section{Genome sequencing and bioinformatics analysis.}

Strain B59 was incubated in ISP2 liquid medium $(50 \mathrm{~mL})$ with seawater at $30^{\circ} \mathrm{C}$ for $30 \mathrm{~h}$. Genomic DNA extraction was performed as described previously ${ }^{1}$. Whole genome sequencing accomplished by Novogene Biotechnology (China) using PacBio Sequel platform. The biosynthetic gene clusters in the genome were analyzed by antiSMASH. ${ }^{2}$ The sequence of $s d l$ cluster was submitted to the GenBank database (MZ150741).

\section{Direct cloning of $s d l$ gene cluster and construction of expression vector}

The pBeloBAC11 vector was used to directly clone the $87-\mathrm{kb} s d l$ gene cluster by means of ExoCET. ${ }^{3}$ The prepared genomic DNA $(50 \mu \mathrm{g})$ above was digested with AvrII-NheI, extracted with phenol-chloroform-isoamyl alcohol (25:24:1, pH 8.0) and precipitated with ethanol. The DNA was dissolved in $\mathrm{ddH}_{2} \mathrm{O}$. The pBeloBAC11 vector carrying homology arms of $s d l$ genes was amplified with PCR (Table S2) using the PrimeSTAR Max DNA Polymerase (Takara). The digested genomic DNA $(10 \mu \mathrm{g})$ and amplified pBeloBAC11 $(1 \mu \mathrm{g})$ were assembled in vitro and then electroporated for homologous recombination. The recombinant plasmid pBAC1610 containing the $s d l$ fragment were identified with ApaLI restriction analysis (Figure S2A). Thereafter it was modified via LCHR (linear 
plus circular homologous recombination) of Red recombineering ${ }^{4}$ to insert the oriT-attP-phiC31 cassette from pR6K-oriT-phiC31 amplified with PCR (Table S2) forming pBAC1611 for targeting in heterologous host's genomes (Figure S2B). ${ }^{1}$

\section{Promoter engineering and in-frame deletion of the sdlR}

For initiating two opposite operons, we constructed a bidirectional promoter cassette, $57 p$-hygkas $O p^{*}$, two promoters ${ }^{5}$ of which were constructed on both ends of hyg in opposite directions by tworound PCR. And then the cassette was inserted into p15A vector (digested with BamHI) by means of ligation with T4 DNA Ligase to afford p15A-cm-57p-hyg-kasOp*.

The 57p-hyg-kas Op* cassette amplified from p15A-cm-57p-hyg-kasOp* with appropriate primers and flanked with $40 \mathrm{bp}$ homologous arms was inserted the upstream of the $s d l D$ in pBAC1611 by LCHR to afford pBAC1612 (Figure S3B).

The sequence between $s d l B$ and $57 p$ in pBAC1612 was partially deleted using $c c d B$ for counterselection ${ }^{6}$ to obtain pBAC1613 (Figure S3C). The target sequence was replaced by the amplified $\mathrm{cm}-\mathrm{ccdB}$ cassette from $\mathrm{p} 15 \mathrm{~A}-\mathrm{cm}-\mathrm{ccdB}$ containing PacI sites at both ends respectively through LCHR in GBred-gyrA462. The recombinant BAC carrying the $c m-c c d B$ was identified by restriction enzyme analysis of colonies selected on LB plates supplemented with chloramphenicol. Thereafter, the correct plasmid was digested with $P a c$ I to release the $c m-c c d B$ cassette, self-ligated with T4 DNA Ligase and electroporated into E. coli GB2005 cells. The recombinant BAC without the $c m-c c d B$ was identified by restriction enzyme analysis of colonies selected on LB plates supplemented with apramycin.

The SA31 promoter ${ }^{7}$ from S. albus $\mathrm{J} 1074$ and $c m-c c d B$ cassette containing AvrII sites at both ends from p15A-cm-ccdB were respectively PCR amplified and were linked by the "bridge PCR" strategy. The resulting fragment with $40 \mathrm{bp}$ homologous arms was inserted in upstream of $s d l J$ in pBAC1613 using LCHR to get pBAC1613-cm-ccdB, which was subsequently modified to be pBAC1614 by removing the $c m-c c d B$ gene using the digestion of $A v r I I$ and ligation of T4 DNA Ligase (Figure S3D).

The $s d l R$ in $\mathrm{pBAC} 1614$ was replaced by the amplified $c m-c c d B$ cassette containing HpaI and PmeI sites at both ends respectively in GBred-gyrA462, and the release of $c m-c c d B$ cassette was performed using the digestion of HpaI/PmeI and ligation of T4 DNA Ligase, to get pBAC1615 (Figure S4B). In order to verify the function of SdIR, we complemented the $s d l R$ gene in trans in pBAC1615. The rpslamp cassette containing HpaI sites at both ends was inserted in the downstream of orfl in pBAC1615 via LCHR (Figure S4C). Then the recombinant plasmid was linearized by HpaI digestion and cotransformed with $21 p$-sdlR, which was obtained by two-round PCR, in GBdir for LLHR to obtain pBAC1616 (Figure S4D).

The correct plasmids identified by restriction digestion was confirmed by sequencing. The primers involved above are listed in Table S2. 


\section{Heterologous expression, fermentation, and isolation of shuangdaolide in S. albus J1074}

The plasmids pBAC1611, pBAC1614, and pBAC1615 were introduced into S. albus J1074, termed S. albus J1074/pBAC1611, S. albus J1074/pBAC1614, and S. albus J1074/pBAC1615 respectively, via conjugation from E. coli ET12567/pUZ8002 according to the literature procedure. ${ }^{10}$

Three independent transconjugants for each plasmid were inoculated into $50 \mathrm{~mL}$ fermentation medium ( $10 \mathrm{~g}$ soluble starch, $0.5 \mathrm{~g} \mathrm{KNO}_{3}, 1 \mathrm{~g}$ yeast extract, $1 \mathrm{~g} \mathrm{NaCl}, 2 \mathrm{~g} \mathrm{CaCO}_{3}$ per liter water) in 250 $\mathrm{mL}$ Erlenmeyer flasks and incubated at $30^{\circ} \mathrm{C}$ for 7 days. Metabolites were extracted with XAD-16 resin and monitored by LC-MS. The crude extracts from $10 \mathrm{~L}$ fermentation of S. albus J1074/pBAC1614 were subjected to normal phase silica gel and eluted with gradient petroleum ether/ethyl acetate mixture to give 15 fractions. The fractions were analyzed by using LC-MS.

Compounds 1 (10 mg from $10 \mathrm{~L}$ of fermentation medium) and 2 (2.8 $\mathrm{mg}$ from $10 \mathrm{~L}$ of fermentation medium) were purified from the fraction $(\mathrm{PE} / \mathrm{EA}=3: 2, \mathrm{v} / \mathrm{v}$ ) by semipreparative HPLC utilizing eluting system $\mathrm{ACN} / \mathrm{H}_{2} \mathrm{O}(45-85 \% \mathrm{ACN})$ with the flow rate of $2.5 \mathrm{~mL} / \mathrm{min}$. Compound 3 ( $4.2 \mathrm{mg}$ from $10 \mathrm{~L}$ of fermentation medium), 4 (3.5 mg from $10 \mathrm{~L}$ of fermentation medium), and 5 (14 mg from $10 \mathrm{~L}$ of fermentation medium) were purified from the fraction $(\mathrm{PE} / \mathrm{EA}=2: 3, \mathrm{v} / \mathrm{v})$ by semipreparative HPLC utilizing eluting system $\mathrm{ACN} / \mathrm{H}_{2} \mathrm{O}(33: 67, \mathrm{v} / \mathrm{v})$ with the flow rate of $2.5 \mathrm{~mL} / \mathrm{min}$.

The crude extracts from $10 \mathrm{~L}$ fermentation of S. albus J1074/pBAC1615 were subjected to the same way as that of S. albus J1074/pBAC1614. Compound 6 (3 mg from $10 \mathrm{~L}$ of fermentation medium) and 7 (4 mg from $10 \mathrm{~L}$ of fermentation medium) were purified from the fraction (PE/EA=1:1, v/v) by semipreparative HPLC utilizing eluting system $\mathrm{ACN} / \mathrm{H}_{2} \mathrm{O}(41: 59, \mathrm{v} / \mathrm{v})$ with the flow rate of $2.5 \mathrm{~mL} / \mathrm{min}$.

Compound 1, yellowish powder, $[\alpha]^{20} \mathrm{D}-11.2\left(c \mathrm{c}\right.$.1, MeOH); IR (KBr) $v_{\max } 3446,2927,2861,1731$, 1650, 1606, 1235, 1033, $984 \mathrm{~cm}^{-1}$; UV(MeOH): $\lambda_{\max } 223 \mathrm{~nm}, 331 \mathrm{~nm}$; HRESIMS: $\mathrm{m} / z$ 569.3106 $[\mathrm{M}+\mathrm{H}]^{+}$(calculated for $\left.\mathrm{C}_{33} \mathrm{H}_{45} \mathrm{O}_{8}, 569.3109\right) ;{ }^{1} \mathrm{H}$ and ${ }^{13} \mathrm{C}$ NMR data, see Table S3.

Compound 2, yellowish powder, $[\alpha]^{20}{ }_{\mathrm{D}}-5.6$ (c 0.1, MeOH); IR (KBr) 3409, 2931, 1700, 1661, 1401, 1257, $970 \mathrm{~cm}^{-1}$; UV(MeOH): $\lambda_{\max } 248 \mathrm{~nm}$; HRESIMS: $m / z$ 593.3065 [M+Na] $]^{+}$(calculated for $\left.\mathrm{C}_{33} \mathrm{H}_{46} \mathrm{O}_{8} \mathrm{Na}, 593.3085\right) ;{ }^{1} \mathrm{H}$ and ${ }^{13} \mathrm{C}$ NMR data, see Table S3.

Compound 3, yellowish powder, $[\alpha]^{20}{ }^{-}-9.4$ (c 0.1, MeOH); IR (KBr) 3359, 2928, 1715, 1653, 1398, 1119, $966 \mathrm{~cm}^{-1}$; UV (MeOH): $\lambda_{\max } 220 \mathrm{~nm}, 246 \mathrm{~nm}$; HRESIMS: $m / z 569.3095[\mathrm{M}+\mathrm{H}]^{+}$(calculated for $\left.\mathrm{C}_{33} \mathrm{H}_{45} \mathrm{O}_{8}, 569.3109\right) ;{ }^{1} \mathrm{H}$ and ${ }^{13} \mathrm{C}$ NMR data, see Table $\mathrm{S} 4$.

Compound 4, yellowish powder, $[\alpha]^{20} \mathrm{D}-18.4$ (c 0.1, MeOH); IR (KBr) 3406, 2964, 1704, 1660, 1403, 1259, 1119, $973 \mathrm{~cm}^{-1}$; UV(MeOH): $\lambda_{\max } 237 \mathrm{~nm}$; HRESIMS: $\mathrm{m} / z 593.3079[\mathrm{M}+\mathrm{Na}]^{+}$(calculated for $\left.\mathrm{C}_{33} \mathrm{H}_{46} \mathrm{O}_{8} \mathrm{Na}, 593.3085\right) ;{ }^{1} \mathrm{H}$ and ${ }^{13} \mathrm{C}$ NMR data, see Table S4.

Compound 6, yellowish powder, $[\alpha]^{20}{ }_{\mathrm{D}}-17.2$ (c 0.1, MeOH); IR (KBr) 3374, 2942, 1706, 1402, 1258 , $967 \mathrm{~cm}^{-1}$; UV(MeOH): $\lambda_{\max } 225 \mathrm{~nm}, 334 \mathrm{~nm}$; HRESIMS: $m / z 555.3319[\mathrm{M}+\mathrm{H}]^{+}$(calculated for $\left.\mathrm{C}_{33} \mathrm{H}_{47} \mathrm{O}_{7}, 555.3316\right) ;{ }^{1} \mathrm{H}$ and ${ }^{13} \mathrm{C}$ NMR data, see Table S5. 
Compound 7, yellowish powder, $[\alpha]^{20}{ }_{\mathrm{D}}-15.7$ (c 0.1, MeOH); IR (KBr) 3393, 2932, 1707, 1400, 1257, $970 \mathrm{~cm}^{-1}$; UV(MeOH): $\lambda_{\max } 228 \mathrm{~nm}, 334 \mathrm{~nm}$; HRESIMS: $m / z 557.3466[\mathrm{M}+\mathrm{H}]^{+}$(calculated for $\left.\mathrm{C}_{33} \mathrm{H}_{49} \mathrm{O}_{7}, 557.3473\right) ;{ }^{1} \mathrm{H}$ and ${ }^{13} \mathrm{C}$ NMR data, see Table S5.

\section{Crystal data for 1.}

The fraction $(\mathrm{PE} / \mathrm{EA}=3: 2, \mathrm{v} / \mathrm{v})$, which was collected from the eluate of normal phase column and mainly contains compound 1 (about $10 \mathrm{mg}$ ), was concentrated in vacuo and re-dissolved in $1.5 \mathrm{~mL}$ $\mathrm{MeOH}$ that was transferred in a $2 \mathrm{~mL}$ clear screw autosampler vial. The lid with holes was then closely screwed on the vial, and Needle-like crystals were obtained after one night at room temperature of about $33^{\circ} \mathrm{C}$.

$\mathrm{C}_{33} \mathrm{H}_{44} \mathrm{O}_{8} \cdot 0.25 \mathrm{H}_{2} \mathrm{O}(M=573.18 \mathrm{~g} / \mathrm{mol})$, monoclinic, space group $\mathrm{P}_{1}, a=5.29650(10) \AA, b=$ 9.8800 (2) $\AA, c=30.1092$ (6) $\AA, \alpha=\gamma=90^{\circ}, \beta=92.745(2)^{\circ}, V=1573.79$ (5) $\AA 3, Z=2, T=100 \mathrm{~K}, \mu$

$(\mathrm{Cu} \mathrm{K \alpha})=0.699 \mathrm{~mm}^{-1}, \rho_{\text {calc }}=1.210 \mathrm{~g} / \mathrm{cm}^{3}, F(000)=617.0 ; 15419$ reflections measured $\left(5.878^{\circ} \leq \Theta \leq\right.$ $\left.134.148^{\circ}\right), 5106$ unique $\left(R_{\text {int }}=0.0422, R_{\text {sigma }}=0.0406\right)$ which were used in all calculations. The final $R_{1}$ was 0.0625 (I $\left.>2 \sigma(\mathrm{I})\right)$ and $w R_{2}$ was 0.1838 (all data); The crystallographic data have been deposited in the Cambridge Crystallographic Data Centre as CCDC 2080925.

\section{Antibacterial assay.}

In vitro activity of compound $\mathbf{5}$ against some bacterial pathogens (Helicobacter pylori G27, Helicobacter pylori 159, Escherichia coli MG1655, Pseudomonas aeruginosa PAO1, Acinetobacter baumannii ATCC19606, Klebsiella neumoniae ATCC35657, Fusobacterium nucleatum, Enterococcus Faecium ATCC19434, Staphylococcus aureus ATCC25923, Staphylococcus aureus USA300, Bacillus cereus, Mycobacterium smegmatis ATCC 607, Candida albicans ATCC SC5314, Candida albicans clinical isolates YY-1-4, Magnaporthe grisea), was assayed. ${ }^{11}$ In brief, the antibacterial ability was evaluated by the minimum inhibitory concentration (MIC) value. The serial dilutions of compound 5 dissolved in DMSO was prepared to get plates with concentrations of 1.75, 3.50, 6.99, 13.99, 27.97, and $55.94 \mu \mathrm{M}$ or $9.72,29.14$, and $87.41 \mu \mathrm{M}$. The bacteria were added into each well except for the negative control (broth only), to give a final concentration of $5 \times 10^{5}$ to $1 \times 10^{6} \mathrm{CFU} / \mathrm{ml}$, After incubation, the plates were detected and the MIC values were calculated.

\section{Cytotoxicity assay.}

The cytotoxicity of compounds $\mathbf{5}$ were tested by MTT assay ${ }^{12}$ with human lung cancer cell line A549, human normal lung (2B) cell, human leukemia cell line kasumi, human breast cancer cell line MDA-MB-231, and RAW246.7 macrophages. 


\section{Supplementary tables:}

Table S1. Plasmids and strains used in this study

\begin{tabular}{|c|c|c|}
\hline Plasmids & Characteristics & Reference \\
\hline pBeloBAC11 & PCR template for direct cloning vector & 1 \\
\hline pR6K-oriT-phiC31 & PCR template of the oriT-attP-phiC31 cassette & 1 \\
\hline p15A-cm-ccdB & PCR template of the $c m-c c d B$ cassette & 6 \\
\hline p15A-cm-57p-hyg-kasOp* & PCR template of the $57 p$-hyg-kasOp* cassette & This study \\
\hline pBAC1610 & pBeloBAC11 harboring $s d l$ gene cluster & This study \\
\hline \multirow[t]{3}{*}{ pBAC1611 } & pBAC1610 was inserted into an oriT-attP- & \\
\hline & phiC31 cassette for targeting in heterologous & This study \\
\hline & host's genomes & \\
\hline pBAC1612 & $\begin{array}{l}\text { pBAC1611 was inserted into an } 57 p \text {-hyg-kasOp* } \\
\text { cassette }\end{array}$ & This study \\
\hline pBAC1613 & $s d l C$ was deleted from $\mathrm{pBAC} 1612$ & This study \\
\hline pBAC1614 & $\begin{array}{l}\text { pBAC } 1613 \text { was inserted into a SA3 } 1 \text { promoter for } \\
\text { overexpression of post-modifying genes }\end{array}$ & This study \\
\hline pBAC1615 & The $s d l R$ of $\mathrm{pBAC} 1614$ was deleted & This study \\
\hline pBAC1616 & $\begin{array}{l}\text { The } s d l R \text { gene was complemented in trans back } \\
\text { to pBAC } 1615\end{array}$ & This study \\
\hline strains & Characteristics & Reference \\
\hline E. coli GB2005 & Host harboring pBeloBAC11 vector & 4 \\
\hline E. coli GBdir & Used to mediate LLHR & 4 \\
\hline E. coli GBred-gyrA462 & CcdB-resistant strain used to mediate LCHR & 6 \\
\hline S. albus $\mathrm{J} 1074$ & Heterologous expression host bacteria & 8 \\
\hline E. coli $\mathrm{ET} 12567 / \mathrm{pUZ} 8002$ & Conjugative transfer donor bacteria & 9 \\
\hline S. albus J1074/pBAC1611 & S. albus J1074 contains plasmid pBAC1611 & This study \\
\hline S. albus J1074/pBAC1614 & S. albus $\mathrm{J} 1074$ contains plasmid pBAC1614 & This study \\
\hline S. albus J1074/pBAC1615 & S. albus J1074 contains plasmid pBAC1615 & This study \\
\hline S. albus J1074/pBAC1616 & S. albus J1074 contains plasmid pBAC1616 & This study \\
\hline
\end{tabular}


Table S2. Primers used in this work

\begin{tabular}{|c|c|c|}
\hline Primer name & Sequence $\left(5^{\prime}-3^{\prime}\right)$ & application \\
\hline \multirow{3}{*}{ BAC-sdl-5 } & acgtcgacggcgaggaaagcgaaagaacttgtttcggaagaggaat & \multirow{6}{*}{ pBAC1610 } \\
\hline & gagaaggaagccetttcatggcetcgecgtagtcaacgCACGC & \\
\hline & TGATAGTCTGATCGACA & \\
\hline \multirow{3}{*}{ BAC-sdl-3 } & ccccattcaactggecgtcetcceggtctccgacaccgaactgccc & \\
\hline & gatgccgtggecctcacccgacgctgtgcagacctagAGGGCA & \\
\hline & CCAATAACTGCCTTAA & \\
\hline sdl-int-apra-5 & tcactggatataccaccgttgatatatcccaatggcatcgATGAA & \multirow{4}{*}{ pBAC1611 } \\
\hline \multirow{3}{*}{ sdl-int-apra-3 } & CGGTTCATGTGCAGC & \\
\hline & ttacgceccgecetgccactcatcgcagtactgttgtaatTGCATC & \\
\hline & CGATGCAAGTGTGT & \\
\hline 57p-hyg-5-1 & TGACCAGGTGTATCCCTCCAGCCGTTCAAC & \multirow{8}{*}{$\begin{array}{l}\text { p15A-57p-hyg- } \\
\text { kasOp* }\end{array}$} \\
\hline & ACTACGATGTATCAGGCGC & \\
\hline $57 \mathrm{p}$-hyg-5-2 & AACCATGGATCCGATGGTATCCTATGACCA & \\
\hline & GGTGTATCCCTCCA & \\
\hline kasOp-hyg-3-1 & GCATGTTGTCAAAGCAGAGACGGTTCGAA & \\
\hline \multirow{3}{*}{ kasOp-hyg-3-2 } & TGTGAACACCGCGGGATGTAAATACTTGAC & \\
\hline & ACCATGGATCCTGGCCACGACTTTACAACA & \\
\hline & CCGCACAGCATGTTGTCAAAGCAGAGACG & \\
\hline sdl-kasOp*-5 & gggcgggcctagcgcgcccgccccgagggcgaagcgagtGAT & \multirow{4}{*}{ pBAC1612 } \\
\hline \multirow{3}{*}{ sdl-kasOp*-3 } & GGTATCCTATGACCAGG & \\
\hline & ggcgatggggacatgctgcgacatcagtggagactccccTGGC & \\
\hline & CACGACTTTACAACAC & \\
\hline sdlB-cmecdB-5 & ccgtgccgccgtggccgctggacatgcttcctccegtgtgTTAAT & \multirow{4}{*}{ pBAC1613 } \\
\hline \multirow{3}{*}{$57 \mathrm{p}-\mathrm{cmccdB}-3$} & 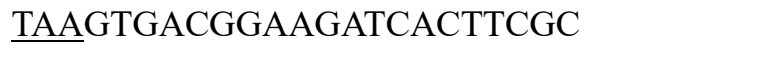 & \\
\hline & tgaacggctggagggatacacctggtcataggataccatcTTAAT & \\
\hline & TAAAGGCGGGCTGGGTTATATTC & \\
\hline hyg-3-out seq & СCTTCACCTTCCTGCACGAC & 57p-hyg-kasOp* \\
\hline hyg-5-out seq & TCGCCGAACAGCTTGATCACC & sequencing primer \\
\hline \multirow[t]{2}{*}{ sdlI-cm-F } & gtttcgaccagtggagaaagatgacgagaagggactgccс $\underline{\mathrm{CCTA}}$ & \multirow{5}{*}{ pBAC1614 } \\
\hline & 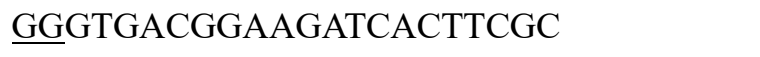 & \\
\hline \multirow{3}{*}{$\begin{array}{l}\text { SA31-AvrII-cm-R } \\
\text { cm-AvrII-SA31-F }\end{array}$} & gtccggCCTAGGAGGCGGGCTGGGTTATATTC & \\
\hline & ccagccegcetCCTAGGCCGGACCTCTCCTCACG & \\
\hline & CTC & \\
\hline
\end{tabular}


sdlJ-SA31-R TtcttctgcgeccctgtccggggaagacatataccttcatGTCA

ATTCCTCCAGCTGACTAC

pBAC1614 sequencing

\begin{tabular}{|c|c|c|}
\hline sdl-SA31-seq & CTTTCGGAATACAGGTAGCC & primer \\
\hline \multirow[t]{2}{*}{ delesdl15-cm F } & ccccagacctccgccgaccccacgaacagggagtgccgaaGTT & \\
\hline & TAAACGTGACGGAAGATCACTTCGC & pBAC1615 \\
\hline \multirow[t]{2}{*}{ delesdl15-cm R } & accegccaccgccagtggcgatgcegcgacacgccgcac $\underline{\mathrm{GTT}}$ & \\
\hline & $\underline{\text { AACAGGCGGGCTGGGTTATATTC }}$ & \\
\hline \multirow[t]{2}{*}{ delesdl15-seq R } & CGTCATGTTCCGGAACGACG & pBAC1615 sequencing \\
\hline & & primer \\
\hline \multirow[t]{2}{*}{ sdlR-compl-amp F } & tctttcgctttcctcgccgtcgacgtcgcccegggcggcGTTAAC & The rpsl-amp cassette \\
\hline & TATCCAATGCTTAATCAGTGAG & was inserted in the \\
\hline \multirow[t]{2}{*}{ sdlR-compl-amp R } & ctgaaagcggctcgggcggcgeccgcgtggtgagcgcgg & downstream of orfl in \\
\hline & $\underline{\text { GTTAACGGTACCGGCCTGGT }}$ & pBAC1615 \\
\hline \multirow[t]{2}{*}{ compl-sdlR F } & tctttcgctttcctcgecgtcgacgtcgcccegggeggcg & \\
\hline & TCAGGCGTGGAGGCCACGA & \\
\hline \multirow[t]{2}{*}{$21 \mathrm{p}$-sdlR R } & GTGCGGGCTCTAACACGTCCTAGTATGGTA & pBAC1616 \\
\hline & GGATGAGCAAAGGGAGTGCCGAAATGATC & \\
\hline \multirow[t]{2}{*}{ compl-sdlR R } & tgaaagcggetcgggeggegccegcgtggtgagegcgggt & \\
\hline & GTGCGGGCTCTAACACGTC & \\
\hline compl-sdlR-seq F & TCTGATTATCGGTCTGGGAC & pBAC1616 sequencing \\
\hline compl-sdlR-seq R & ACGGCGATCTACAACCGCA & primer \\
\hline
\end{tabular}


Table S3. ${ }^{1} \mathrm{H}$ and ${ }^{13} \mathrm{C}$ NMR Data of $\mathbf{1 - 2}$

\begin{tabular}{|c|c|c|c|c|}
\hline \multirow[b]{2}{*}{ no. } & \multicolumn{2}{|r|}{$\mathbf{1}^{b}$} & \multicolumn{2}{|r|}{$2^{c}$} \\
\hline & $\delta_{\mathrm{C}}$ & $\delta_{\mathrm{H}}(J$ in $\mathrm{Hz})$ & $\delta_{\mathrm{C}}$ & $\delta_{\mathrm{H}}(J$ in $\mathrm{Hz})$ \\
\hline 1 & $172.2, \mathrm{C}$ & & $169.8, \mathrm{C}$ & \\
\hline \multirow[t]{2}{*}{2} & $34.8, \mathrm{CH}_{2}$ & $3.11, \mathrm{dd}(8.8,14.9)$ & $33.9, \mathrm{CH}_{2}$ & $2.85, \mathrm{~d}(7.2)$ \\
\hline & & $2.96, \mathrm{dd}(6.8,14.9)$ & & \\
\hline 3 & $118.9, \mathrm{CH}$ & $5.46, \mathrm{dd}(6.8,8.8)$ & $116.1, \mathrm{CH}$ & $5.33, \mathrm{t}(7.2)$ \\
\hline 4 & $139.5, \mathrm{C}$ & & 141.3, C & \\
\hline 5 & $71.2, \mathrm{CH}$ & $4.82, \mathrm{~d}(9.2)$ & $71.7, \mathrm{CH}$ & $4.78, \mathrm{~d}(8.4)$ \\
\hline 6 & $140.0, \mathrm{CH}$ & $5.77, \mathrm{~d}(9.2)$ & $135.0, \mathrm{CH}$ & $5.45, \mathrm{~d}(8.4)$ \\
\hline 7 & $134.8, \mathrm{C}$ & & 131.1, C & \\
\hline 8 & $147.8, \mathrm{CH}$ & $6.60, \mathrm{~d}(15.0)$ & $137.7, \mathrm{CH}$ & $6.13, \mathrm{~d}(15.3)$ \\
\hline 9 & $122.5, \mathrm{CH}$ & $6.47, \mathrm{dd}(10.9,15.0)$ & $127.4, \mathrm{CH}$ & $5.40, \mathrm{dd}(9.8,15.3)$ \\
\hline 10 & $146.5, \mathrm{CH}$ & 7.08, d (10.9) & $50.1, \mathrm{CH}$ & 2.93, d (9.8) \\
\hline 11 & 130.0, C & & $143.4, \mathrm{C}$ & \\
\hline 12 & 198.8, C & & $148.8, \mathrm{C}$ & \\
\hline 13 & 74.2, $\mathrm{CH}$ & $3.06, \mathrm{dd}(5.2,8.2)$ & 199.9, C & \\
\hline 14 & $135.2, \mathrm{CH}$ & $5.68, \mathrm{dd}(8.2,15.5)$ & $51.0, \mathrm{CH}$ & $2.88, \mathrm{~d}(10.9)$ \\
\hline 15 & $132.9, \mathrm{CH}$ & $5.48, \mathrm{dd}(7.7,15.5)$ & $123.6, \mathrm{CH}$ & $5.01, \mathrm{t}(10.9)$ \\
\hline 16 & $56.1, \mathrm{CH}$ & $2.92, \mathrm{dd}(1.9,7.7)$ & $139.1, \mathrm{CH}$ & $5.55, \mathrm{dd}(7.0,10.9)$ \\
\hline 17 & $57.8, \mathrm{CH}$ & $2.88, \mathrm{~m}$ & $63.6, \mathrm{CH}$ & $4.24, \mathrm{~m}$ \\
\hline \multirow[t]{2}{*}{18} & $34.5, \mathrm{CH}_{2}$ & $2.27, \mathrm{~m}$ & $39.0, \mathrm{CH}_{2}$ & $1.35, \mathrm{~m}$ \\
\hline & & $1.34, \mathrm{~m}$ & & $1.13, \mathrm{~m}$ \\
\hline 19 & $76.5, \mathrm{CH}$ & $5.16, \mathrm{~m}$ & $74.1, \mathrm{CH}$ & $4.98, \mathrm{~m}$ \\
\hline 20 & $37.3, \mathrm{CH}$ & $1.93, \mathrm{~m}$ & $32.1, \mathrm{CH}$ & $2.06, \mathrm{~m}$ \\
\hline 21 & $76.3, \mathrm{CH}$ & $3.69, \mathrm{dd}(2.2,9.5)$ & 79.0, CH & $3.32, \mathrm{~m}^{a}$ \\
\hline 22 & $31.3, \mathrm{CH}$ & $1.55, \mathrm{~m}^{a}$ & $31.3, \mathrm{CH}$ & $1.39, \mathrm{~m}^{a}$ \\
\hline \multirow[t]{2}{*}{23} & $29.0, \mathrm{CH}_{2}$ & $1.67, \mathrm{~m}^{a}$ & $29.2, \mathrm{CH}_{2}$ & $1.55, \mathrm{~m}^{a}$ \\
\hline & & $1.55, \mathrm{~m}^{a}$ & & $1.39, \mathrm{~m}^{a}$ \\
\hline \multirow[t]{2}{*}{24} & $34.3, \mathrm{CH}_{2}$ & $1.77, \mathrm{~m}$ & $33.3, \mathrm{CH}_{2}$ & $1.60, \mathrm{~m}^{a}$ \\
\hline & & $1.67, \mathrm{~m}^{a}$ & & $1.55, \mathrm{~m}^{a}$ \\
\hline 25 & 110.6, C & & 105.1, C & \\
\hline \multirow[t]{2}{*}{26} & $130.8, \mathrm{CH}$ & $5.65, \mathrm{dt}(2.3,6.0)$ & $37.3, \mathrm{CH}_{2}$ & $1.72, \mathrm{~m}^{a}$ \\
\hline & & & & $1.60, \mathrm{~m}^{a}$ \\
\hline 27 & $129.9, \mathrm{CH}$ & $6.06, \mathrm{dt}(1.4,6.0)$ & $23.3, \mathrm{CH}_{2}$ & $1.72, \mathrm{~m}^{a}$ \\
\hline
\end{tabular}




\begin{tabular}{lllll}
\hline 28 & $74.3, \mathrm{CH}_{2}$ & $4.54, \mathrm{dd}(1.4,2.3)$ & $65.7, \mathrm{CH}_{2}$ & $3.70, \mathrm{~m}$ \\
& & & $3.64, \mathrm{~m}$ \\
29 & $14.7, \mathrm{CH}_{3}$ & $1.78, \mathrm{~s}$ & $11.9, \mathrm{CH}_{3}$ & $1.55, \mathrm{~s}$ \\
30 & $12.5, \mathrm{CH}_{3}$ & $1.85, \mathrm{~s}$ & $12.8, \mathrm{CH}_{3}$ & $1.72, \mathrm{~s}$ \\
31 & $12.0, \mathrm{CH}_{3}$ & $1.93, \mathrm{~s}$ & $12.5, \mathrm{CH}_{3}$ & $1.80, \mathrm{~s}$ \\
32 & $9.3, \mathrm{CH}_{3}$ & $0.85, \mathrm{~d}(6.8)$ & $6.8, \mathrm{CH}_{3}$ & $0.80, \mathrm{~d}(7.2)$ \\
33 & $17.0, \mathrm{CH}_{3}$ & $0.80, \mathrm{~d}(6.0)$ & $17.4, \mathrm{CH}_{3}$ & $0.76, \mathrm{~d}(5.7)$ \\
$13-\mathrm{OH}$ & & $4.28, \mathrm{~d}(5.2)$ & & \\
\hline
\end{tabular}

${ }^{a}$ overlapped

${ }^{b}$ measured at $600 \mathrm{MHz}$ for ${ }^{1} \mathrm{H} \mathrm{NMR}$ and at $150 \mathrm{MHz}$ for ${ }^{13} \mathrm{C}$ NMR in $\mathrm{CDCl}_{3}-d$

${ }^{c}$ measured at $500 \mathrm{MHz}$ for ${ }^{1} \mathrm{H}$ NMR and at $125 \mathrm{MHz}$ for ${ }^{13} \mathrm{C}$ NMR in DMSO- $d_{6}$ 
Table S4. ${ }^{1} \mathrm{H}$ and ${ }^{13} \mathrm{C}$ NMR Data of 3-4

\begin{tabular}{|c|c|c|c|c|}
\hline \multirow[b]{2}{*}{ no. } & \multicolumn{2}{|r|}{$\mathbf{3}^{b}$} & \multicolumn{2}{|r|}{$4^{c}$} \\
\hline & $\delta_{\mathrm{C}}$ & $\delta_{\mathrm{H}}(J$ in $\mathrm{Hz})$ & $\delta_{\mathrm{C}}$ & $\delta_{\mathrm{H}}(J$ in $\mathrm{Hz})$ \\
\hline 1 & 171.6, C & & $170.8, \mathrm{C}$ & \\
\hline \multirow[t]{2}{*}{2} & $33.5, \mathrm{CH}_{2}$ & $3.12, \mathrm{dd}(8.1,17.8)$ & $33.2, \mathrm{CH}_{2}$ & $3.11, \mathrm{dd}(7.7,16.0)$ \\
\hline & & $2.98, \mathrm{dd}(7.6,17.8)$ & & $2.96, \mathrm{dd}(8.4,16.0)$ \\
\hline 3 & $118.3, \mathrm{CH}$ & 5.54, dd $(7.6,8.1)$ & $115.9, \mathrm{CH}$ & 5.49, dd $(7.7,8.4)$ \\
\hline 4 & $140.2, \mathrm{C}$ & & 141.2, C & \\
\hline 5 & $73.5, \mathrm{CH}$ & $4.96, \mathrm{~d}(8.7)$ & $72.2, \mathrm{CH}$ & $4.82, \mathrm{dd}(3.6,8.6)$ \\
\hline 6 & $132.9, \mathrm{CH}$ & $5.54, \mathrm{~d}(8.7)$ & $133.9, \mathrm{CH}$ & $5.44, \mathrm{~d}(8.6)$ \\
\hline 7 & $133.8, \mathrm{C}$ & & $131.5, \mathrm{C}$ & \\
\hline 8 & $137.8, \mathrm{CH}$ & $6.25, \mathrm{~d}(15.4)$ & $136.3, \mathrm{CH}$ & $6.19, \mathrm{~d}(15.3)$ \\
\hline 9 & $128.5, \mathrm{CH}$ & $5.29, \mathrm{dd}(9.7,15.4)$ & $129.4, \mathrm{CH}$ & $5.30, \mathrm{dd}(9.7,15.3)$ \\
\hline 10 & $51.2, \mathrm{CH}$ & $2.96, \mathrm{~d}(9.7)$ & $50.3, \mathrm{CH}$ & $2.84, \mathrm{~d}(9.7)$ \\
\hline 11 & $146.3, \mathrm{C}$ & & $144.5, \mathrm{C}$ & \\
\hline 12 & $148.1, \mathrm{C}$ & & $148.5, \mathrm{C}$ & \\
\hline 13 & $201.5, \mathrm{C}$ & & 201.4, C & \\
\hline 14 & $50.9, \mathrm{CH}$ & $3.06, \mathrm{~d}(10.3)$ & $49.4, \mathrm{CH}$ & $3.09, \mathrm{~d}(10.6)$ \\
\hline 15 & $126.3, \mathrm{CH}$ & $5.18, \mathrm{t}(10.3)$ & $125.9, \mathrm{CH}$ & $5.08, \mathrm{t}(10.6)$ \\
\hline 16 & $138.2, \mathrm{CH}$ & $5.74, \mathrm{dd}(7.6,10.6)$ & $137.8, \mathrm{CH}$ & $5.57, \mathrm{dd}(7.6,10.6)$ \\
\hline 17 & $68.6, \mathrm{CH}$ & $4.47, \mathrm{~m}$ & $65.1, \mathrm{CH}$ & $4.24, \mathrm{~m}$ \\
\hline \multirow[t]{2}{*}{18} & $42.5, \mathrm{CH}_{2}$ & $1.68, \mathrm{~m}^{a}$ & $43.5, \mathrm{CH}_{2}$ & $1.50, \mathrm{~m}^{a}$ \\
\hline & & $1.53, \mathrm{~m}^{a}$ & & 1.36, ddd $(1.6,6.4,7.9)$ \\
\hline 19 & 71.7, $\mathrm{CH}$ & $3.65, \mathrm{~m}$ & $67.3, \mathrm{CH}$ & $3.79, \mathrm{~m}$ \\
\hline 20 & $40.4, \mathrm{CH}$ & $1.53, \mathrm{~m}^{a}$ & $37.9, \mathrm{CH}$ & $1.50, \mathrm{~m}^{a}$ \\
\hline 21 & $79.9, \mathrm{CH}$ & $4.86, \mathrm{dd}(5.1,6.2)$ & $79.3, \mathrm{CH}$ & $4.79, \mathrm{dd}(2.5,9.4)$ \\
\hline 22 & $33.4, \mathrm{CH}$ & $1.93, \mathrm{~m}$ & $32.2, \mathrm{CH}$ & $1.85, \mathrm{~m}^{a}$ \\
\hline \multirow[t]{2}{*}{23} & 29.6, $\mathrm{CH}_{2}$ & $1.68, \mathrm{~m}^{a}$ & $23.9, \mathrm{CH}_{2}$ & $1.69, \mathrm{~m}$ \\
\hline & & $1.35, \mathrm{~m}$ & & $1.21, \mathrm{~m}$ \\
\hline \multirow[t]{2}{*}{24} & 25.6, $\mathrm{CH}_{2}$ & $2.73, \mathrm{~m}$ & $37.1, \mathrm{CH}_{2}$ & $2.56, \mathrm{~m}$ \\
\hline & & $2.54, \mathrm{~m}$ & & $2.49, \mathrm{~m}$ \\
\hline 25 & 156.1, C & & 199.7, C & \\
\hline 26 & $105.2, \mathrm{CH}$ & $5.97, \mathrm{~d}(3.2)$ & 131.6, CH & $6.13, \mathrm{dq}(1.6,15.6)$ \\
\hline 27 & $110.4, \mathrm{CH}$ & $6.26, \mathrm{dd}(1.7,3.2)$ & $142.6, \mathrm{CH}$ & $6.86, \mathrm{dq}(6.8,15.6)$ \\
\hline 28 & $141.0, \mathrm{CH}$ & $7.28, \mathrm{~d}(1.7)$ & $17.9, \mathrm{CH}_{3}$ & $1.85, \mathrm{dd}(1.6,6.8)$ \\
\hline
\end{tabular}




\begin{tabular}{lllll}
\hline 29 & $12.7, \mathrm{CH}_{3}$ & $1.72, \mathrm{~s}$ & $11.6, \mathrm{CH}_{3}$ & $1.58, \mathrm{~s}$ \\
30 & $13.1, \mathrm{CH}_{3}$ & $1.75, \mathrm{~s}$ & $12.7, \mathrm{CH}_{3}$ & $1.73, \mathrm{~s}$ \\
31 & $12.9, \mathrm{CH}_{3}$ & $1.92, \mathrm{~s}$ & $12.4, \mathrm{CH}_{3}$ & $1.74, \mathrm{~s}$ \\
32 & $9.5, \mathrm{CH}_{3}$ & $0.78, \mathrm{~d}(6.8)$ & $8.9, \mathrm{CH}_{3}$ & $0.53, \mathrm{~d}(6.7)$ \\
33 & $16.5, \mathrm{CH}_{3}$ & $0.92, \mathrm{~d}(6.8)$ & $16.7, \mathrm{CH}_{3}$ & $0.78, \mathrm{~d}(6.8)$ \\
$5-\mathrm{OH}$ & & & $4.94, \mathrm{~d}(3.7)$ \\
$12-\mathrm{OH}$ & & & $9.12, \mathrm{brs}$ \\
$17-\mathrm{OH}$ & & & $4.86, \mathrm{~d}(5.0)$ \\
$19-\mathrm{OH}$ & & & $4.12, \mathrm{~d}(4.5)$ \\
\hline
\end{tabular}

${ }^{a}$ overlapped

${ }^{b}$ measured at $600 \mathrm{MHz}$ for ${ }^{1} \mathrm{H} \mathrm{NMR}$ and at $150 \mathrm{MHz}$ for ${ }^{13} \mathrm{C}$ NMR in $\mathrm{CDCl}_{3}-d$

${ }^{c}$ measured at $600 \mathrm{MHz}$ for ${ }^{1} \mathrm{H}$ NMR and at $150 \mathrm{MHz}$ for ${ }^{13} \mathrm{C}$ NMR in DMSO- $d_{6}$ 
Table S5. ${ }^{1} \mathrm{H}(500 \mathrm{MHz})$ and ${ }^{13} \mathrm{C}(125 \mathrm{MHz}) \mathrm{NMR}$ Data of 6-7 in $\mathrm{CDCl}_{3}-d$

\begin{tabular}{|c|c|c|c|c|}
\hline \multirow[b]{2}{*}{ no. } & \multicolumn{2}{|r|}{6} & \multicolumn{2}{|r|}{7} \\
\hline & $\delta_{\mathrm{C}}$ & $\delta_{\mathrm{H}}(J$ in $\mathrm{Hz})$ & $\delta_{\mathrm{C}}$ & $\delta_{\mathrm{H}}(J$ in $\mathrm{Hz})$ \\
\hline 1 & $172.4, \mathrm{C}$ & & $172.4, \mathrm{C}$ & \\
\hline 2 & $33.9, \mathrm{CH}_{2}$ & $3.13, \mathrm{dd}(8.1,16.0)$ & $33.9, \mathrm{CH}_{2}$ & $3.13, \mathrm{dd}(8.1,16.0)$ \\
\hline & & $3.07, \mathrm{dd}(7.4,16.0)$ & & $3.07, \mathrm{dd}(7.4,16.0)$ \\
\hline 3 & $117.1, \mathrm{CH}$ & $5.69, \mathrm{dd}(7.4,8.1)$ & 117.1, CH & 5.69, dd $(7.4,8.1)$ \\
\hline 4 & $140.3, \mathrm{C}$ & & $140.3, \mathrm{C}$ & \\
\hline 5 & $71.8, \mathrm{CH}$ & $4.86, \mathrm{~d}^{a}$ & $71.8, \mathrm{CH}$ & $4.85, \mathrm{~d}^{a}$ \\
\hline 6 & $139.2, \mathrm{CH}$ & $5.62, \mathrm{~d}(9.1)$ & 139.3, CH & $5.62, \mathrm{~d}(9.2)$ \\
\hline 7 & $134.9, \mathrm{C}$ & & $134.8, \mathrm{C}$ & \\
\hline 8 & $146.6, \mathrm{CH}$ & $6.49, \mathrm{~d}(15.2)$ & $146.7, \mathrm{CH}$ & $6.49, \mathrm{~d}(15.2)$ \\
\hline 9 & $123.3, \mathrm{CH}$ & $6.48, \mathrm{dd}(11.5,15.2)$ & $123.2, \mathrm{CH}$ & $6.48, \mathrm{dd}(11.4,15.2)$ \\
\hline 10 & 144.6, CH & $7.20, \mathrm{~d}(11.5)$ & 144.6, CH & $7.20, \mathrm{~d}(11.4)$ \\
\hline 11 & $131.2, \mathrm{C}$ & & $131.2, \mathrm{C}$ & \\
\hline 12 & 199.3, C & & 199.2, C & \\
\hline 13 & $75.0, \mathrm{CH}$ & $5.16, \mathrm{dd}(3.8,9.7)$ & 75.0, $\mathrm{CH}$ & 5.16, dd $(3.2,9.8)$ \\
\hline 14 & $130.8, \mathrm{CH}$ & $5.36, \mathrm{dd}(9.7,15.2)$ & $130.8, \mathrm{CH}$ & $5.36, \mathrm{dd}(9.8,15.1)$ \\
\hline 15 & $135.4, \mathrm{CH}$ & $6.42, \mathrm{dd}(10.5,15.2)$ & $135.4, \mathrm{CH}$ & $6.42, \mathrm{dd}(10.5,15.1)$ \\
\hline 16 & $132.6, \mathrm{CH}$ & $6.11, \mathrm{dd}(10.5,15.3)$ & $132.6, \mathrm{CH}$ & $6.10, \mathrm{dd}(10.5,15.1)$ \\
\hline 17 & $132.2, \mathrm{CH}$ & 5.78, ddd $(4.7,10.1,15.3)$ & $132.2, \mathrm{CH}$ & 5.78, ddd $(4.7,10.0,15.1)$ \\
\hline 18 & $39.8, \mathrm{CH}_{2}$ & $2.38, \mathrm{~m}$ & $39.7, \mathrm{CH}_{2}$ & $2.37, \mathrm{~m}^{a}$ \\
\hline & & $2.22, \mathrm{~m}$ & & $2.23, \mathrm{~m}^{a}$ \\
\hline 19 & $72.8, \mathrm{CH}$ & $3.81, \mathrm{~m}$ & $72.8, \mathrm{CH}$ & $3.80, \mathrm{~m}$ \\
\hline 20 & $38.8, \mathrm{CH}$ & $1.78, \mathrm{~m}^{a}$ & $38.7, \mathrm{CH}$ & $1.75, \mathrm{~m}^{a}$ \\
\hline 21 & $80.8, \mathrm{CH}$ & $4.86, \mathrm{dd}^{a}$ & $80.7, \mathrm{CH}$ & $4.85, \mathrm{dd}^{a}$ \\
\hline 22 & $34.2, \mathrm{CH}$ & $1.78, \mathrm{~m}^{a}$ & $34.1, \mathrm{CH}$ & $1.75, \mathrm{~m}^{a}$ \\
\hline 23 & $25.6, \mathrm{CH}_{2}$ & $1.67, \mathrm{~m}^{a}$ & $25.2, \mathrm{CH}_{2}$ & $1.63, \mathrm{~m}$ \\
\hline & & $1.26, \mathrm{~m}$ & & $1.22, \mathrm{~m}$ \\
\hline 24 & $37.0, \mathrm{CH}_{2}$ & $2.50, \mathrm{~m}$ & $39.7, \mathrm{CH}_{2}$ & $2.37, \mathrm{~m}^{a}$ \\
\hline & & $2.41, \mathrm{~m}$ & & $2.23, \mathrm{~m}^{a}$ \\
\hline 25 & $200.0, \mathrm{C}$ & & $210.9, \mathrm{C}$ & \\
\hline 26 & $131.9, \mathrm{CH}$ & $6.07, \mathrm{dq}(1.5,15.7)$ & $44.9, \mathrm{CH}_{2}$ & $2.32, \mathrm{t}(7.4)$ \\
\hline 27 & $142.7, \mathrm{CH}$ & $6.81, \mathrm{dq}(6.8,15.7)$ & $17.5, \mathrm{CH}_{2}$ & $1.56, \mathrm{~m}$ \\
\hline 28 & $18.4, \mathrm{CH}_{3}$ & $1.88, \mathrm{dd}(1.5,6.8)$ & $13.9, \mathrm{CH}_{3}$ & $0.89, \mathrm{t}(7.4)$ \\
\hline 29 & $14.4, \mathrm{CH}_{3}$ & $1.69, \mathrm{~s}$ & $14.4, \mathrm{CH}_{3}$ & $1.68, \mathrm{~s}$ \\
\hline 30 & $12.8, \mathrm{CH}_{3}$ & $1.89, \mathrm{~s}$ & $12.8, \mathrm{CH}_{3}$ & $1.89, \mathrm{~s}$ \\
\hline 31 & $12.0, \mathrm{CH}_{3}$ & $1.93, \mathrm{~s}$ & $12.0, \mathrm{CH}_{3}$ & $1.93, \mathrm{~s}$ \\
\hline 32 & $7.2, \mathrm{CH}_{3}$ & $0.83, \mathrm{~d}(6.3)$ & 7.2, $\mathrm{CH}_{3}$ & $0.82, \mathrm{~d}(6.8)$ \\
\hline 33 & $16.0, \mathrm{CH}_{3}$ & $0.84, \mathrm{~d}(6.0)$ & $15.9, \mathrm{CH}_{3}$ & $0.82, \mathrm{~d}(6.8)$ \\
\hline $13-\mathrm{OH}$ & & $4.27, \mathrm{~d}(3.8)$ & & $4.28, \mathrm{~d}(3.2)$ \\
\hline
\end{tabular}


Table S6. Putative functions of the genes in the $s d l$ gene cluster

\begin{tabular}{|c|c|c|c|c|}
\hline Gene & Size $^{\mathrm{a}}$ & Predicted Protein Function & homolog & $\begin{array}{c}\text { Identity/ } \\
\text { Similarity (\%) }\end{array}$ \\
\hline orf1 & 363 & peptidase M23 & (WP_037928733.1) & $94 / 96$ \\
\hline orf 2 & 232 & putative endonuclease & (WP_123620351.1) & $83 / 88$ \\
\hline sdlA & 224 & $\begin{array}{l}\text { 4'-phosphopantetheinyl } \\
\text { transferase }\end{array}$ & CppF (ABV83217.1) & $52 / 64$ \\
\hline$s d l B$ & 409 & cytochrome P450 & TamI (ADC79647.1) & $50 / 66$ \\
\hline$s d l C$ & 113 & $\begin{array}{c}\text { DUF4180 domain-containing } \\
\text { protein }\end{array}$ & (WP_004936897.1) & $96 / 97$ \\
\hline$s d l D$ & 7785 & type I polyketide synthase & & \\
\hline SdlE & 4558 & type I polyketide synthase & & \\
\hline$S d l F$ & 2821 & type I polyketide synthase & & \\
\hline$S d l G$ & 2991 & type I polyketide synthase & & \\
\hline$S d l H$ & 3071 & type I polyketide synthase & & \\
\hline$s d l I$ & 453 & 2-nitropropane dioxygenase & BatK (ADD82952.1) & $52 / 72$ \\
\hline$s d l J$ & 373 & $\begin{array}{c}\text { malonyl CoA-acyl carrier } \\
\text { protein transacylase }\end{array}$ & OocW (AFX60345.1) & $51 / 67$ \\
\hline$s d l K$ & 419 & $\begin{array}{c}\text { hydroxymethylglutaryl-CoA } \\
\text { synthase }\end{array}$ & BatC (ADD82944.1) & $68 / 82$ \\
\hline$s d l L$ & 261 & enoyl-CoA hydratase/isomerase & BatD (ADD82945.1) & $56 / 68$ \\
\hline$s d l M$ & 250 & enoyl-CoA hydratase & BatE (ADD82946.1) & $63 / 78$ \\
\hline$s d l N$ & 81 & acyl carrier protein & BatA (ADD82942.1) & $46 / 64$ \\
\hline$s d l O$ & 415 & Beta-ketoacyl synthase & BatB (ADD82943.1) & $58 / 72$ \\
\hline$s d l P$ & 321 & $\begin{array}{c}\text { malonyl CoA-acyl carrier } \\
\text { protein transacylase }\end{array}$ & BatH (ADD82949.1) & $34 / 51$ \\
\hline$s d l Q$ & 301 & SDR family oxidoreductase & NcmD (ARS01471.1) & $40 / 58$ \\
\hline$s d l R$ & 1299 & SDR family oxidoreductase & (WP_004936953.1) & $91 / 93$ \\
\hline
\end{tabular}




$$
\text { orf3 } 551 \quad \text { multicopper oxidase family }
$$

protein

a Size indicates the number of amino acids 
Table S7. Predicted KS substrates in shuangdaolide biosynthesis by the TransATor ${ }^{13}$. (Black: correct prediction; red: incorrect prediction.)

\begin{tabular}{ccc}
\hline KS domain & Predicted substrate specificity & Actual substrate \\
\hline KSLM & various starters & acetylStarter \\
KS1 & $\beta$ L-OH & AcStarter \\
KS2 & $\beta$-keto or double bonds & double bonds \\
KS3 & $\beta$ L-OH & $\beta$ OH \\
KS4 & $\alpha$ Me reduced/keto/D-OH & $\alpha$ Me reduced \\
KS5 & $\beta$ D-OH & $\alpha$ Me $\beta$ OH \\
KS6 & $\beta$ D-OH & $\beta$ OH \\
KS7 & double bonds (mostly e-configured) & double bonds \\
KS8 & double bonds (mostly e-configured) & non-elongating (double bonds) \\
KS9 & double bonds (mostly e-configured) & double bonds \\
KS10 & $\beta$ L-OH & $\beta$ OH \\
KS11 & exomethylene & beta branch \\
KS12 & double bonds (mostly e-configured) & double bonds \\
KS13 & exomethylene & beta branch \\
KS14 & $\alpha$ Me reduced/keto/D-OH & $\alpha$ Me $\beta$ OH \\
KS15 & non-elongating (double bonds) & non-elongating (shifted double \\
& & bonds) \\
\hline
\end{tabular}


Table S8. Predicted stereospecificities of KR domains in the shuangdaolide biosynthesis.

\begin{tabular}{|c|c|c|c|c|}
\hline Ketoreductase & $\begin{array}{l}\text { KR specificity } \\
\text { code }\end{array}$ & KR type & $\begin{array}{c}\text { Predicted } \\
\text { Stereochemistry }\end{array}$ & $\begin{array}{c}\text { Observed } \\
\text { Stereochemistry } \\
\text { (position of -OH) }\end{array}$ \\
\hline KR1 & GVMGG & A type (L) & - & - \\
\hline KR2 & GVQGQ & A type (L) & $(S)$ & $S(25)$ \\
\hline KR3 & LTLLD & B type (D) & - & - \\
\hline KR4 & FGLVD & B type (D) & $(R)$ & $R(21)$ \\
\hline KR5 & GVLRD & B type (D) & $(S)$ & $S(19)$ \\
\hline KR7 & GLTRD & B type (D) & - & - \\
\hline KR8 & GAADG & C type ${ }^{a}$ & - & - \\
\hline KR9 & GVAER & A type (L) & $(S)$ & $S(13)$ \\
\hline KR11 & GVIQD & B type (D) & - & - \\
\hline KR13 & IVLLD & B type (D) & $(R)$ & $R(5)$ \\
\hline KR14 & GVLHD & B type (D) & - & - \\
\hline
\end{tabular}

a $\mathrm{C}$ type $\mathrm{KR}$ domain lacks the key conserved tyrosine residue (in YAAAN motif). 


\section{Supplementary figures:}

A

\begin{tabular}{|c|c|}
\hline$n$ & ACRFP \\
\hline KS1 & I AV I GMSGRFP \\
\hline KS2 & VAIIGMSGRYP \\
\hline KS3 & I AV I GMSGRFP \\
\hline KS4 & VAVIGMSGRFG \\
\hline KS5 & VAI VGMSARFP \\
\hline KS6 & VAVI GMSGRFP \\
\hline KS7 & I AV I GL AGRYP \\
\hline KS8 & - A I I GL AGRYP \\
\hline KSg & $|A| \mid G L A G R Y P$ \\
\hline KS10 & I AVI GMSGRFP \\
\hline KS11 & VAVVGMSGRYP \\
\hline KS12 & I AVI I GMGGRYP \\
\hline KS13 & I AVVGMSGRYP \\
\hline KS14 & I AVVGMSGRFA \\
\hline KS15 & I AV I GL AGRYP \\
\hline
\end{tabular}

B

KR1 GVYLLSGGA

KR2 GTYLITGGC

- VYVIVGGA

GVYVVLGGA

GVYLI ITGGA

GVYLVAGGA

GTYL I TGGT

GVYLVTGGM

GVYVVIGGA
GVYLVTGGL
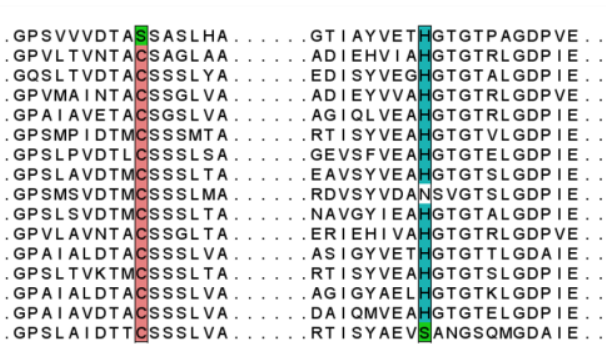

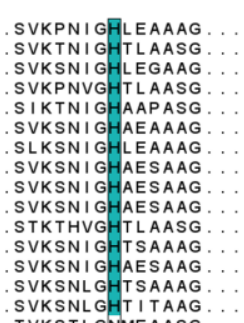

AHVVLEEAP VHVVVES - AHV I IEEY -
AHMVVESR AHVVVEEAP AHVVIEEY. AHVVVAEY AHVVVEEYVHVVMAEY AHL VLEEYVHMVVESYP AHVVLEEYAHVIVGEY: AHLVLEEP AHLVVEEY.

FHGAGVMG TTGVMDVDWADFEGVLAPKTHGTVHLDELTAEDPLDFFLAFS SIAAYVGDFGQGS YAAANRFLD I HAAGVQGQGSVLEKDLPTFEDVLDPK IRGTLTLDEAVAGEELDFVCYFSSTAAVI GDFGTCDYAVGNRFOM VHSALTLLDQSVARMPEERFRAAVRAKVD I SVRI AQVFADEPLDFALFFS STNSFHRS AGQSNYVSGCVFKD
VQSVFGLV I HGAGVLRDGFLAHKGAEELREVLAPKTAGTLNLDVASKDDALDFFALFSSTAAVTGNVGQADYAAANAFLD LHAAGL TRDAFVVKKDADEFALVLAPKVAGL VHLDEATQDADLDFL VAFSS VAGALGNVGQADYAAANAFMD I HGAGAADGAL I VDTPDRDSLDGLAATASGVVNLDAATRSLPLDFFVTFSSPHGVDGGVGQVDGAAAHAFLN VHAAGVAERGSLLSADVRS FQDVL SPKVDGTRVLDDVLAAEPLDFLCHFSSASAVLGDFGGCAY SVANRFQT VHSAGVI QDDF I LRKSEQEFREVLAPKVSGLVSLDEATADLDLDFFVTFSSGAAVTGNVGQADYAAANAFMD VHSAIVLLDRSLERMDERRFRAAVTAKVDVSVRLAQVFRGEPLDFLLFFSSMNSFLKASGQCNYVAGSVFED VHAAGVLHDGLVRGKTAEECRAVLAPKVAGL VNLDEASKDVPLDFLVSFSGAAGVLGNAGOSDYAAANAFLD.

C

\begin{tabular}{|c|c|}
\hline & - EWVDGY \\
\hline & LAT \\
\hline & VAHLELARTAHAAFT \\
\hline H6 & AAYLEMARAALDASR \\
\hline & YTGEEFFLR \\
\hline 78 & LEMAVAASCLS \\
\hline 111 & AYLEMALAAAADSR \\
\hline & VACLEMARAAAALT \\
\hline
\end{tabular}

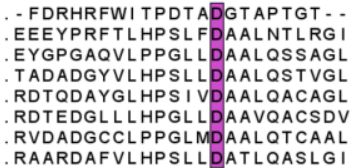

D $\quad$ CMT3

CMT3 - - VYRDCESATALNEL VAESVAARVEVLLARDPAHRVRIFE

CMT4 VEGIYKRNMIADYFNDVVGDCVVALVESRLARDPGATLRIL

I GSGSGGTSEKVLRRL APYRDSLAEYCYTDVSQYFLRNG

源

E $A C P L M$

- - LRGL VSGVLKLAADE I HPRDRL SDLGCDSVGNTLLALRLGEHYGI DVAPSFFYGYFTLEMVRDRLLE EYLRDRL SQVFR I PKARLDAAAEL GEYG I ES I Q I MELMRLL TKDFD - NLPGTLFFEYRT I GEL AEYFTE- MRRI VSGAVGLSPDRL YEDDNL ADYGFDSVSL AGLATGLGEHWGVEVTPALFYSRSTLRKLREYFAT- VI VERLQTSLGLDRRE I SEEDSFADYGVDS I LGVELTQQLSTDLG I SL SVTDLFDHSSVRAL ARYVVEH DYLKELVARTLLI IPAGSIDAREQLSAYGID -...-SAVIGLPVAKIDAQAPLTTYGI - MVDFLASELRVPAERIDADDALEQYGI - ALRTA IV VIPLARVDTRAPFETFGLDSVMAMTMT ARMEAVFG - ALPPTLFFEFGTPRELARHLVA- ELRAGLAAVLVPREQIHADENLSELGFDSVSLVKLARALGDSLAIEVLPSVFFSHPTPEKLVAHL I AE - ELRAGL AAVLFL SESEVDPRRNFVELGLDS I I GVEWVKS I NKAYGTSLTATRL YDYPS VMELAAHL - . - LTTLLSEVLKVPARRI EPGSSLERYGIDSI QAVNLTARLEASFG - LLPKTLFFEYRNIKALSRYFT - - ELTASL ADALFLLEEE I DQDTKFTDI GLDS I VGVEWMMNA I NKRYGLDLTVTRLYDHPT VVEL SAYV - - - RLROTLSEVIKI DRSQ I AADDAFMDYGVDS I I GVRL I QE I NRALGTDL AT TDL FDHGSVSRLARY I... ... ITAVVAELLGMRTDELDPATPLEDLGFSSLHSTQLLQALRAEVDPTADLGALSECRSTRDLLR .....

Figure S1. Sequence alignment of shuangdaolide PKSs. ${ }^{14}$

A Multiple alignment of KS domains. Catalytic triad is highlighted. In KSLM, the cysteine (in TAC motif) is missing, indicating the loss of transthioesterification. In KS8 and KS15, the first histidine (in EA $\underline{H} G T G$ motif) is missing, hinting at its inactivity as a decarboxylase.

B Multiple alignments of KR domains. The first highlighted column determines the stereospecificity of KR-generated hydroxyl groups. (Table S7) The other three highlighted columns are catalytic triad. KS8 lacks the tyrosine (in $\underline{Y} A A A N$ motif) implying to its inactivity.

C Multiple alignments of DH domains. Conserved motif is highlighted. In DHLM and DH8, the catalytic histidine (in HxxxGxxxx motif) is absent, indicating its inactivity.

D Multiple alignments of cMT domains. The SAM-binding site was highlighted.

E Multiple alignments of ACP domains. The essential serine residue is highlighted in green. the conserved tryptophan residue for $\beta$-branching is marked in blue. 
A

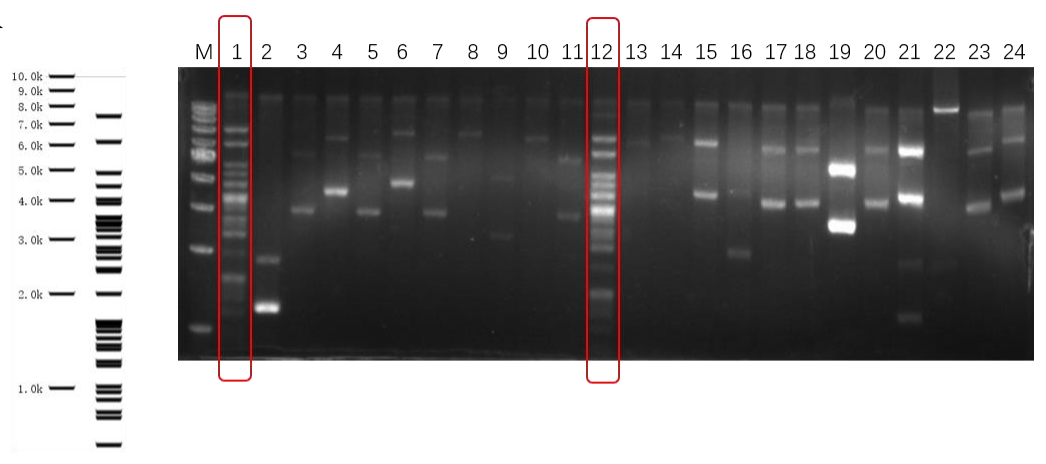

B

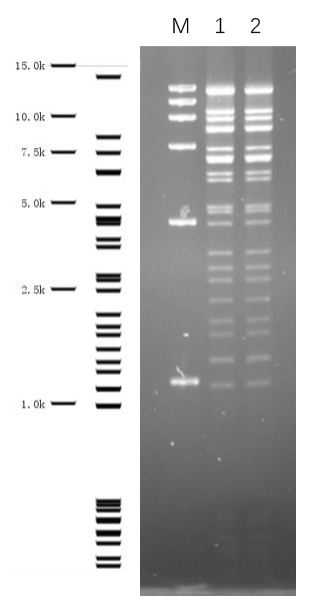

Figure S2. Restriction analysis of pBAC1610 (A) and pBAC1611 (B).

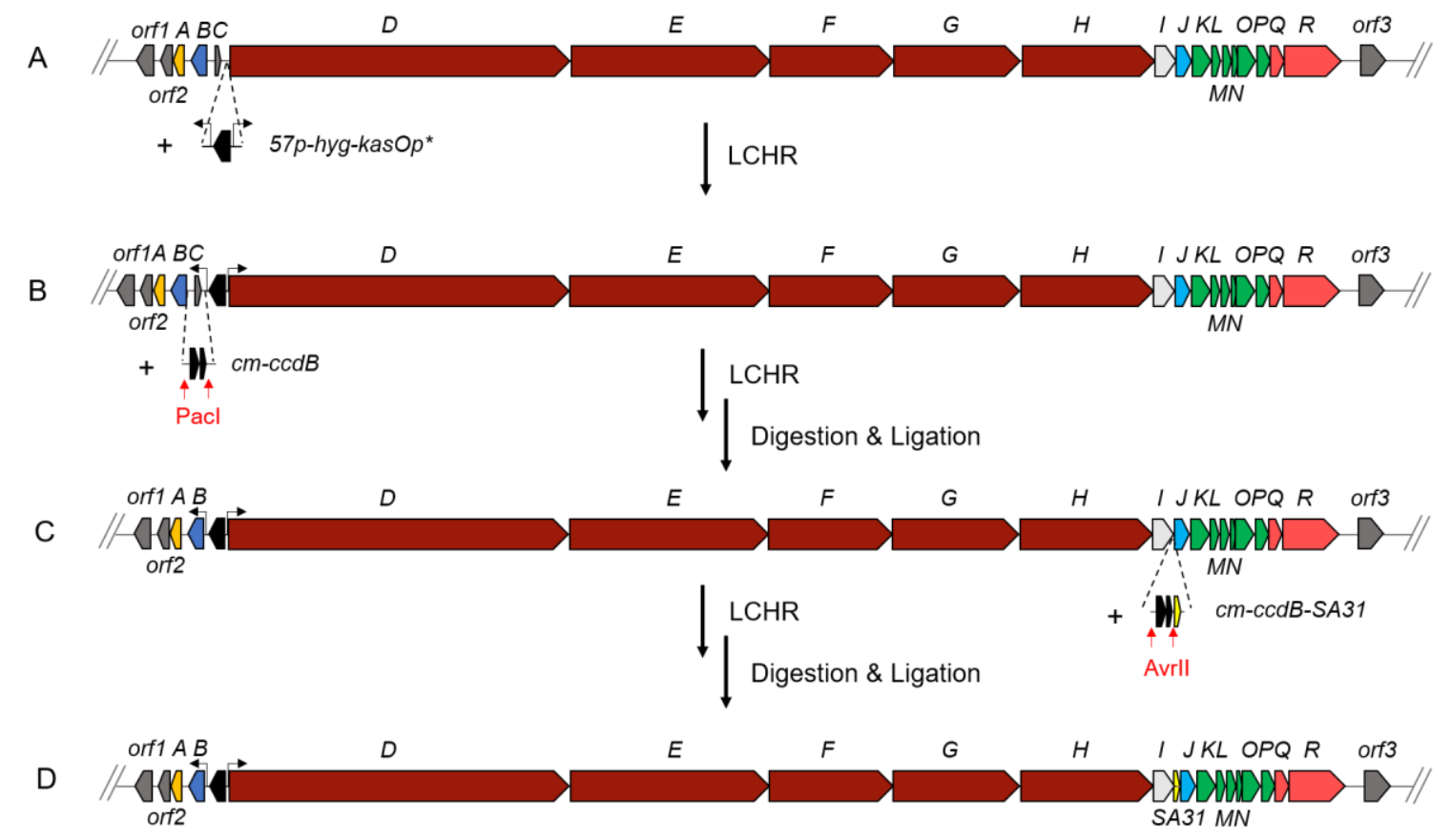

Figure S3. the promoter optimization of $s d l$ gene cluster. (A) pBAC1611; (B) pBAC1612; (C) pBAC1613; (D) pBAC1614. 


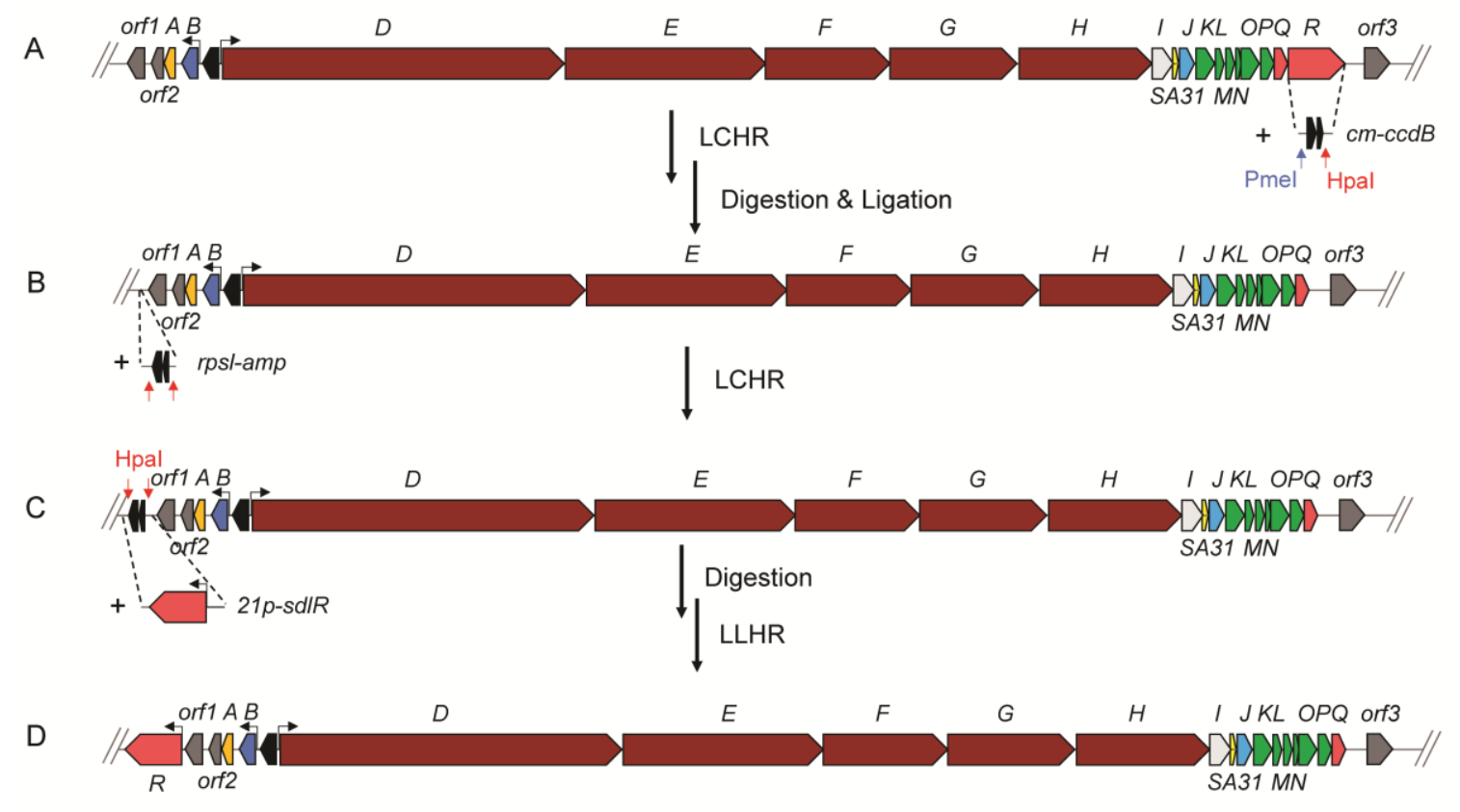

Figure S4. In-frame deletion and trans-complement of the sdlR gene. (A) pBAC1614; (B) pBAC1615; (D) pBAC1616.

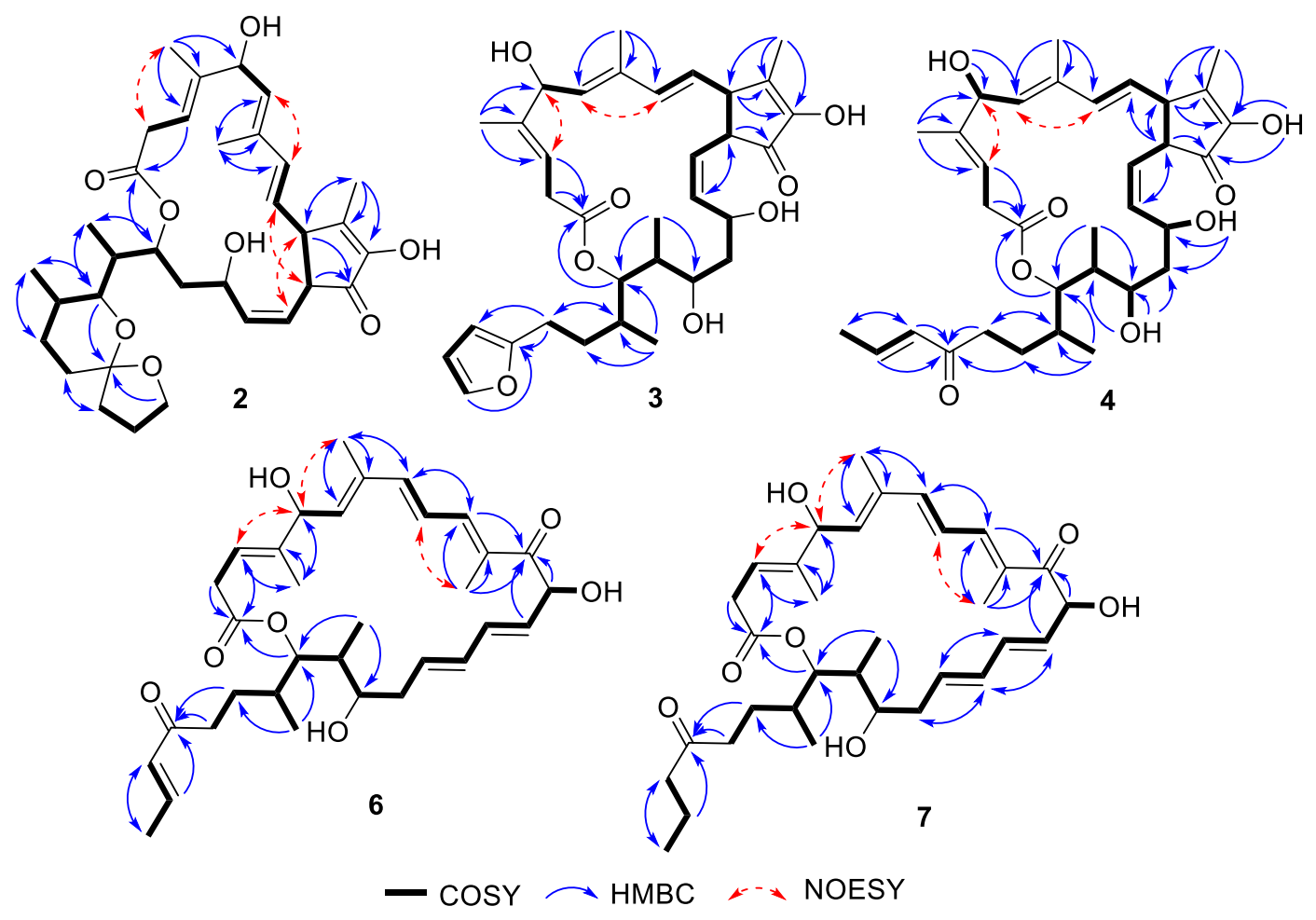


Figure S5. Key COSY, HMBC, and NOESY correlations of 2-4 and 6-7.

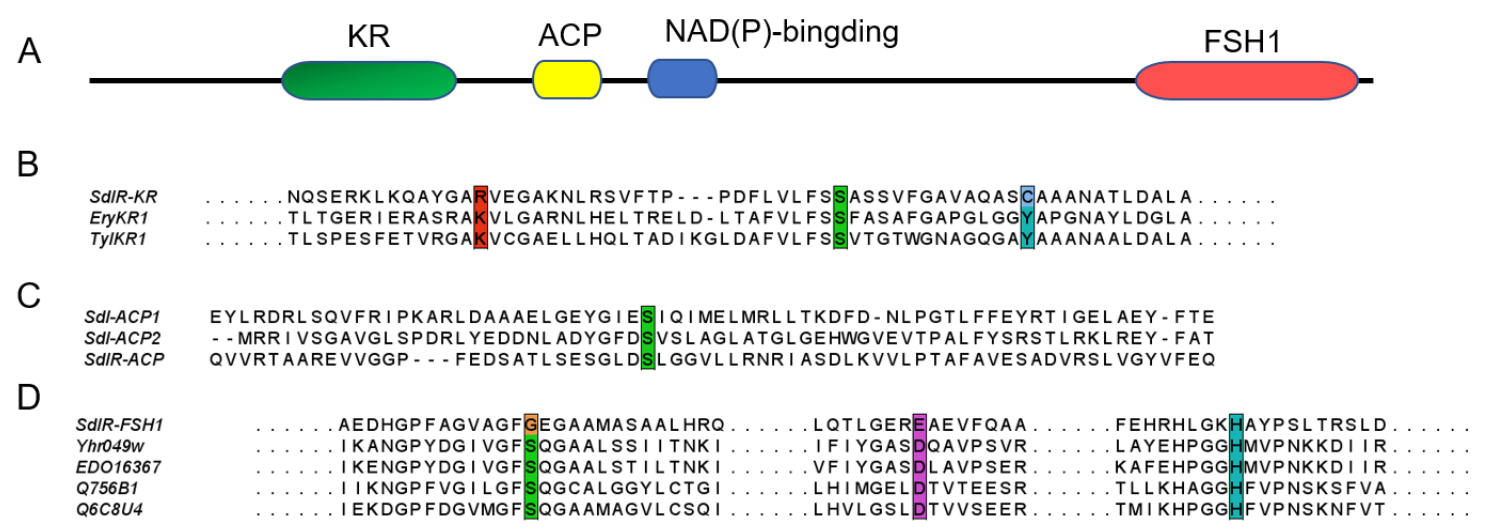

Figure S6. The conserved domains analysis of SdIR via NCBI. ${ }^{15}$

A The conserved domains distributed in SdlR.

B Multiple alignments of KR domains. Catalytic triad is highlighted. The most important catalytic

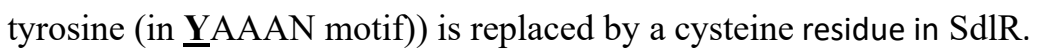

C Multiple alignments of ACP domains. The essential serine residue is highlighted in green.

D Multiple alignments of serine hydrolase (FSH1) domains. The three highlighted columns are catalytic triad. In SdlR, the cysteine (in GF $\underline{\mathbf{S}} \mathbf{Q}$ motif) is missing.

A<smiles>[R]C=CC=CC(O)C(=O)C(C)=C[R]</smiles>

B

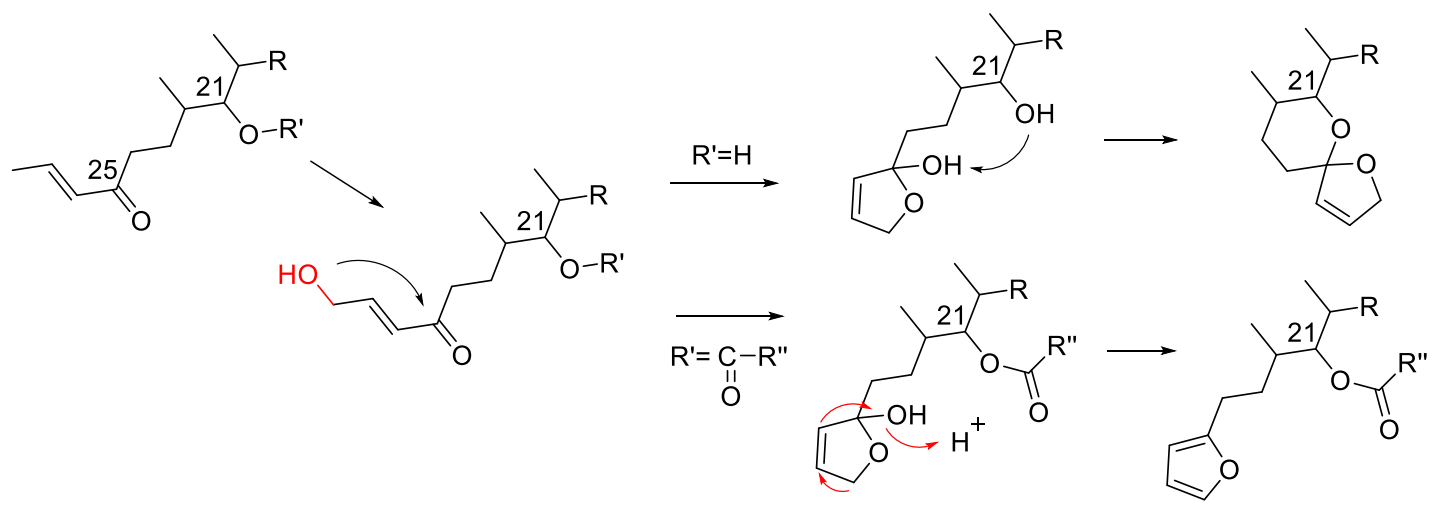


Figure S7. Presumed post-modification of shuangdaolides.

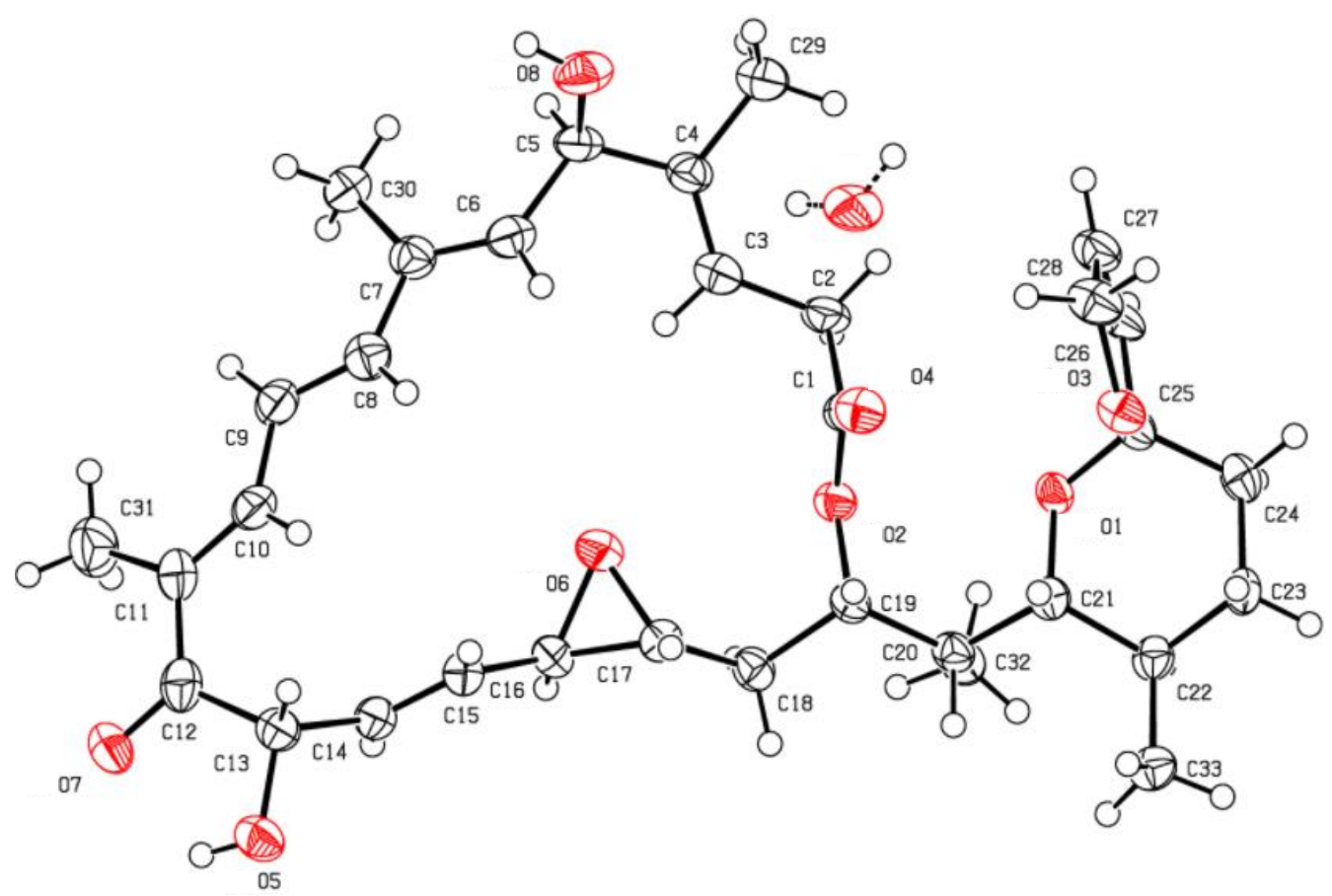

Figure S8. The X-ray structure of $\mathbf{1}$ with the ellipsoid contour at 50\% probability levels. 


\section{Reference}

(1) Wang, H.; Li, Z.; Jia, R.; Hou, Y.; Yin, J.; Bian, X.; Li, A.; Müller, R.; Stewart, A. F.; Fu, J.; Zhang, Y. Nat. Protoc. 2016, 1, 1175-1190.

(2) Weber, T.; Blin, K.; Duddela, S.; Krug, D.; Kim, H. U.; Bruccoleri, R.; Lee, S. Y.; Fischbach, M. A.; Müller, R.; Wohlleben, W.; Breitling, R.; Takano, E.; Medema, M. H. Nucleic Acids Res. 2015. 43, W237- W243.

(3) Wang, H.; Li, Z.; Jia, R.; Yin, J.; Li, A.; Xia, L.; Yin, Y.; Müller, R.; Fu, J.; Stewart, A. F.; Zhang, Y. Nucleic Acids Res. 2018, 46, e28.

(4) Fu, J.; Bian, X.; Hu, S.; Wang, H.; Huang, F.; Seibert, P. M.; Plaza, A.; Xia, L.; Müller, R.; Stewart, A. F.; Zhang, Y. Nat. Biotechnol. 2012, 30, 440-446.

(5) (a) Siegl, T.; Tokovenko, B.; Myronovskyi, M.; Luzhetskyy, A. Metab. Eng. 2013, 19, 98-106. (b) Bai, C.; Zhang, Y.; Zhao, X.; Hu, Y.; Xiang, S.; Miao, J.; Lou, C.; Zhang, L. Proc. Natl. Acad. Sci. U. S. A. 2015, 112, 12181-12186.

(6) Wang, H.; Bian, X.; Xia, L.; Ding, X.; Müller, R.; Zhang, Y.; Fu, J.; \& Stewart, A. F. Nucleic Acids Res. 2014, 42, e37.

(7) Luo, Y.; Zhang, L.; Barton, K. W.; \& Zhao, H. ACS. Synth. Biol. 2015, 4, 1001-1010.

(8) Chater, K. F.; Wilde, L. C. J. Bacteriol. 1976, 128, 644-650.

(9) Bierman, M.; Logan, R.; Brien, K. O.; Seno, E. T.; Rao, R. N.; Schoner, B. E. Gene. 1992, 116, $43-49$.

(10) Keiser, T.; Bibb, M. J.; Buttner, M. J.; Charter, K. F.; Hopwood, D. A. Practical Streptomycetes Genetics, The John Innes Foundation: Norwich, UK, 2000.

(11) Huang, Y.; Hang, X.; Jiang, X.; Zeng, L.; Jia, J.; Xie, Y.; Li, F.; Bi, H. Antimicrob. Agents. Chemother. 2019, 63, e00004- e00019.

(12) Mosmann, T. J. Immunol. Methods. 1983, 65, 55-63.

(13) Helfrich, E.; Ueoka, R.; Dolev, A.; Rust, M.; Meoded, R. A.; Bhushan, A.; Califano, G.; Costa, R.; Gugger, M.; Steinbeck, C.; Moreno, P.; Piel, J. Nat. Chem. Biol. 2019, 15, 813-821.

(14) (a) Keatinge-Clay, A. T. Nat. Prod. Rep. 2012, 29, 1050-1073. (b) Piasecki, S. K.; Zheng, J.; Axelrod, A. J.; Detelich, M. E.; Keatinge-Clay, A. T. Proteins. 2014, 82, 2067-2677. 


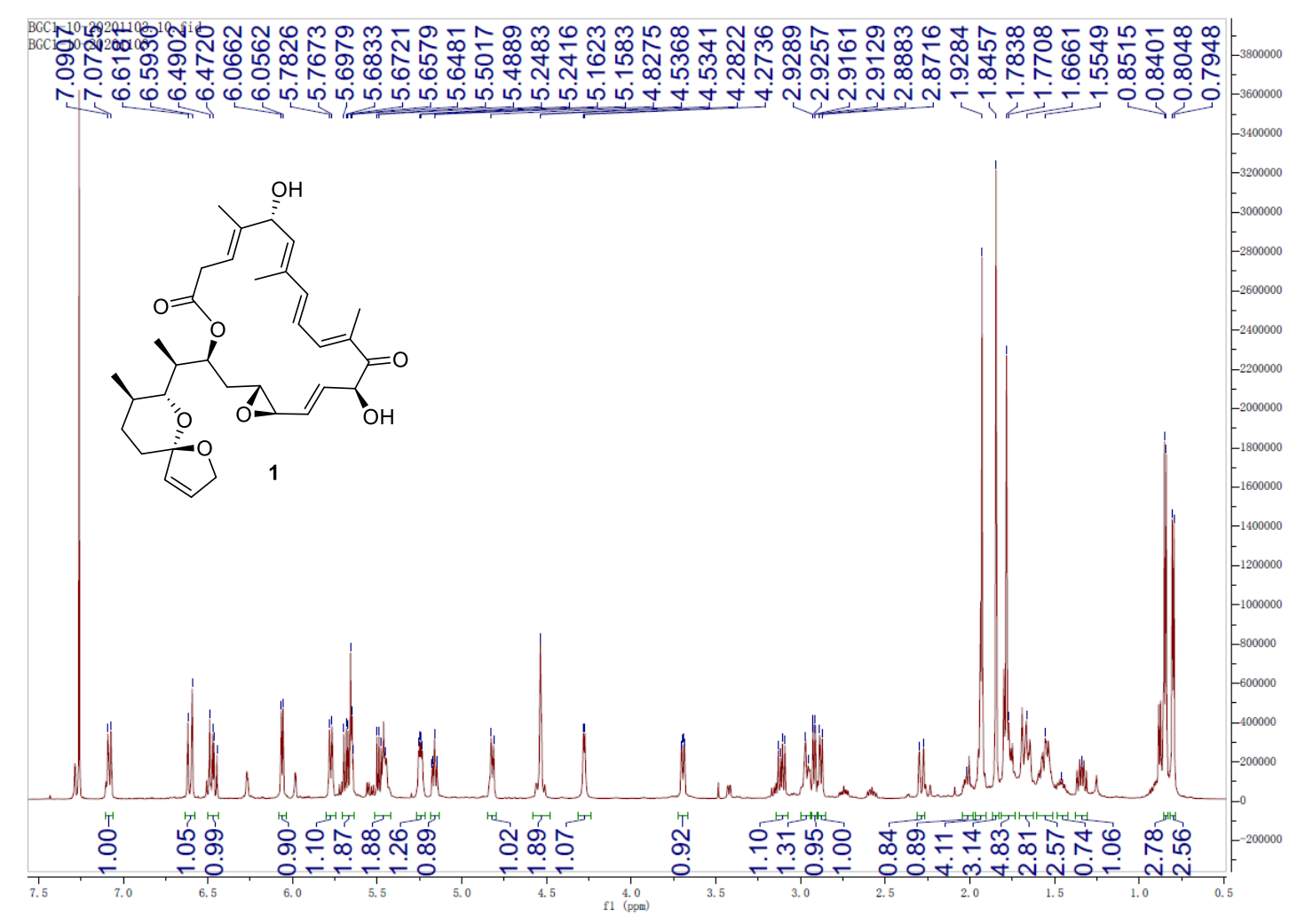

Figure S9. ${ }^{1} \mathrm{H}$ NMR spectrum $(600 \mathrm{MHz})$ of shuangdaolide $\mathrm{A}$ (1) in $\mathrm{CDCl}_{3}$.

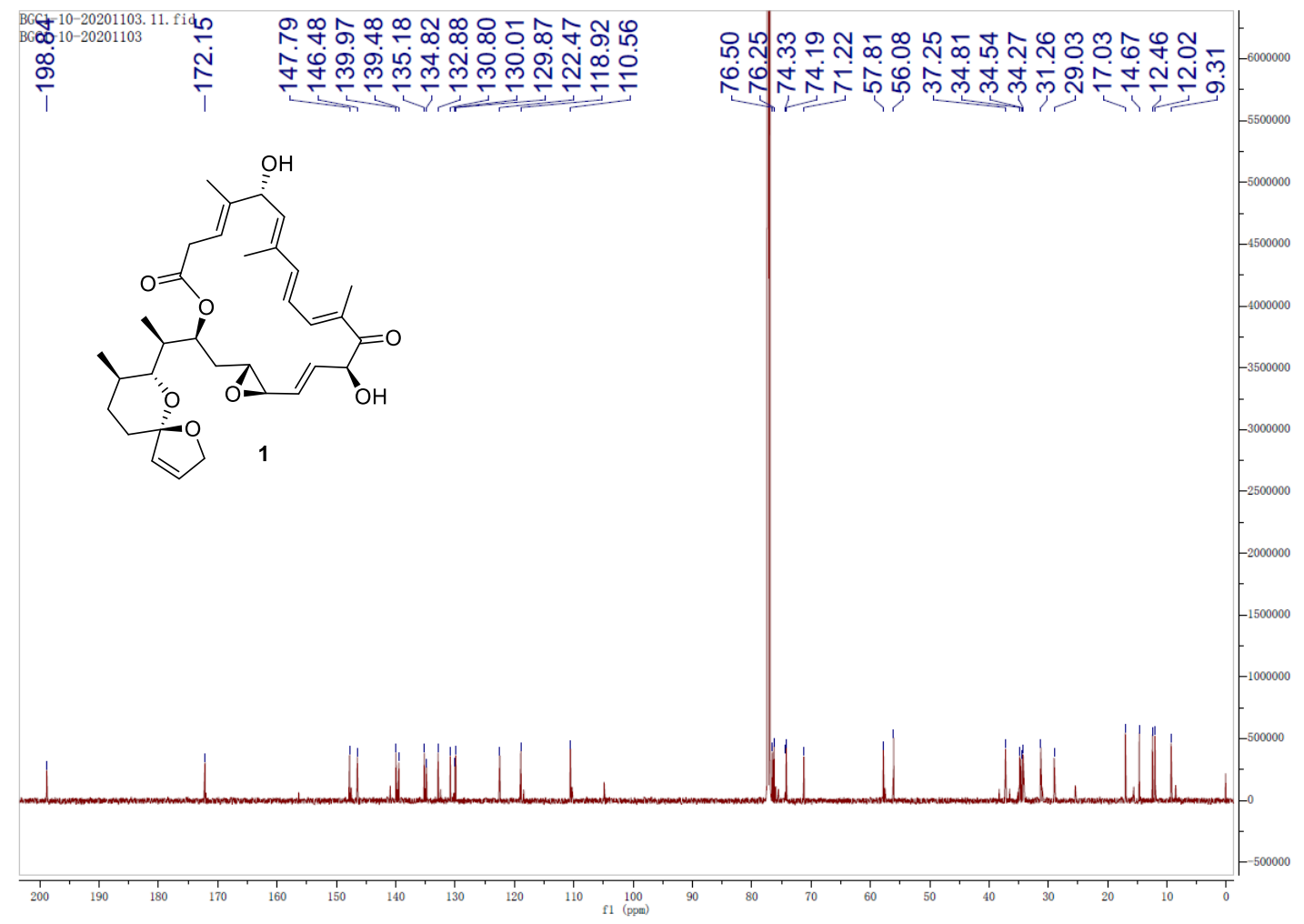

Figure S10. ${ }^{13} \mathrm{C}$ NMR spectrum $(150 \mathrm{MHz})$ of shuangdaolide $\mathrm{A}(1)$ in $\mathrm{CDCl}_{3}$. 


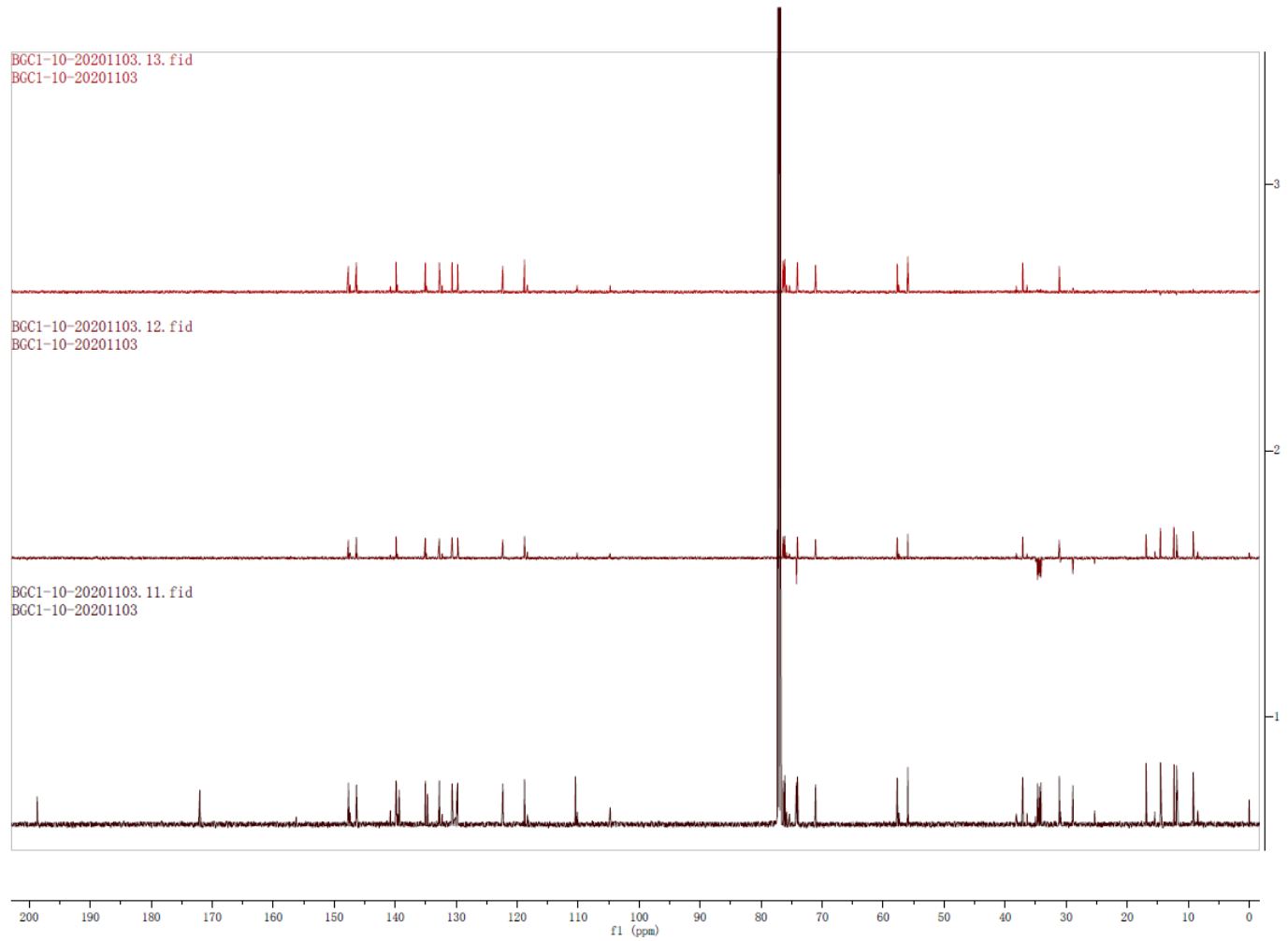

Figure S11. DEPT spectrum (150 MHz) of shuangdaolide A (1) in $\mathrm{CDCl}_{3}$.

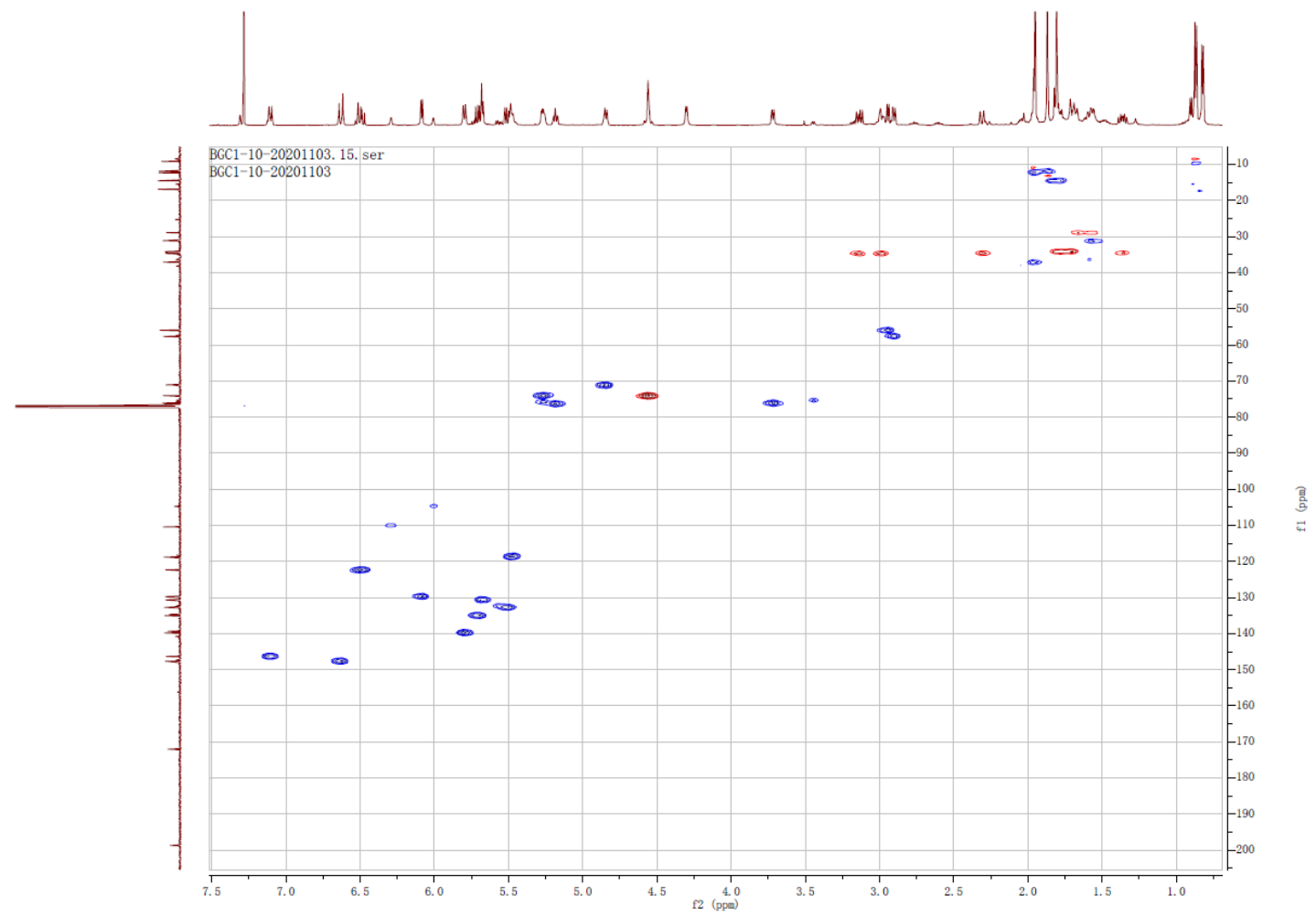

Figure S12. HSQC spectrum (150 MHz/600 MHz) of shuangdaolide A (1) in $\mathrm{CDCl}_{3}$. 


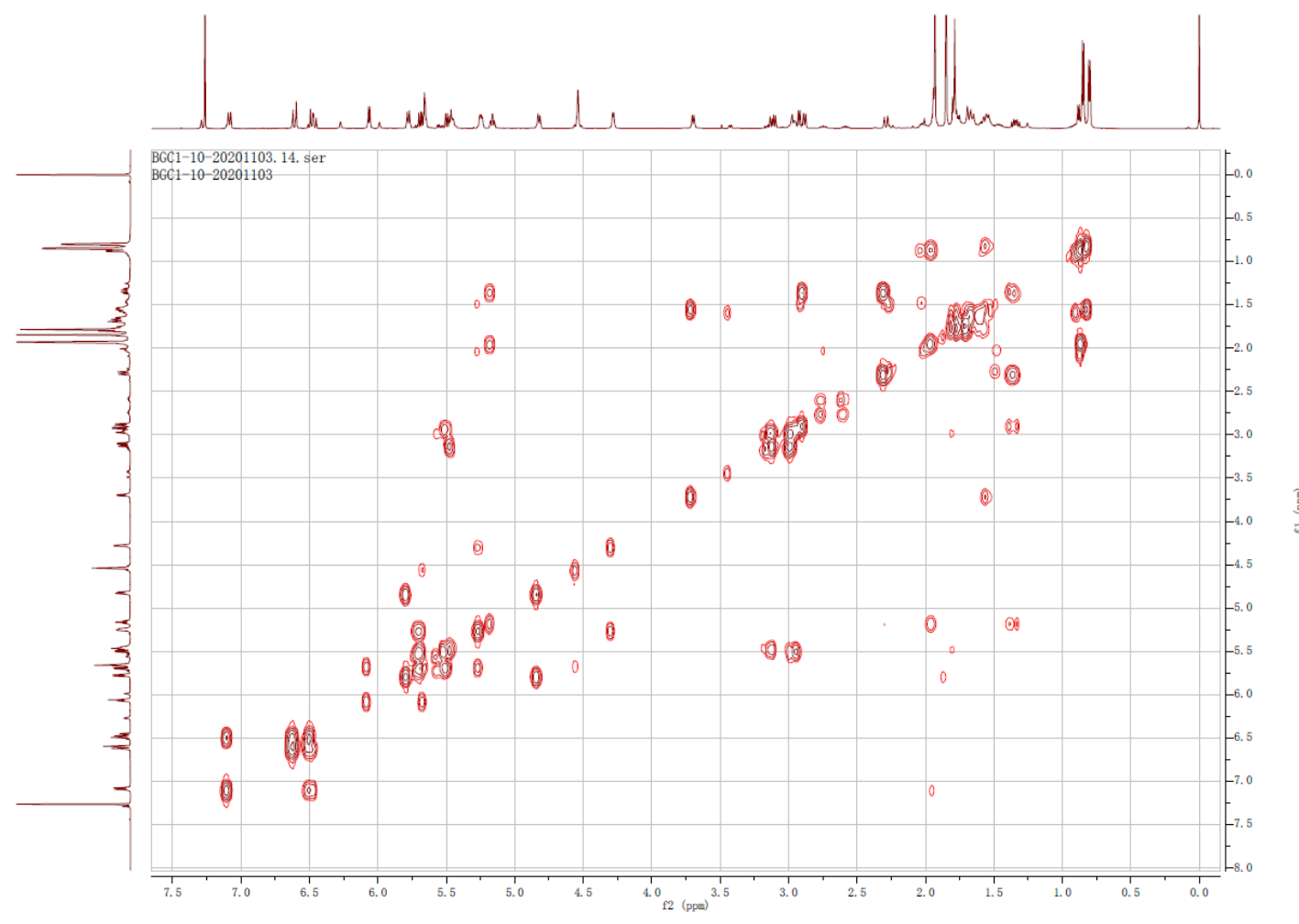

Figure S13. ${ }^{1} \mathrm{H}-{ }^{1} \mathrm{H}$ COSY spectrum $(600 \mathrm{MHz})$ of shuangdaolide $\mathrm{A}(1)$ in $\mathrm{CDCl}_{3}$.

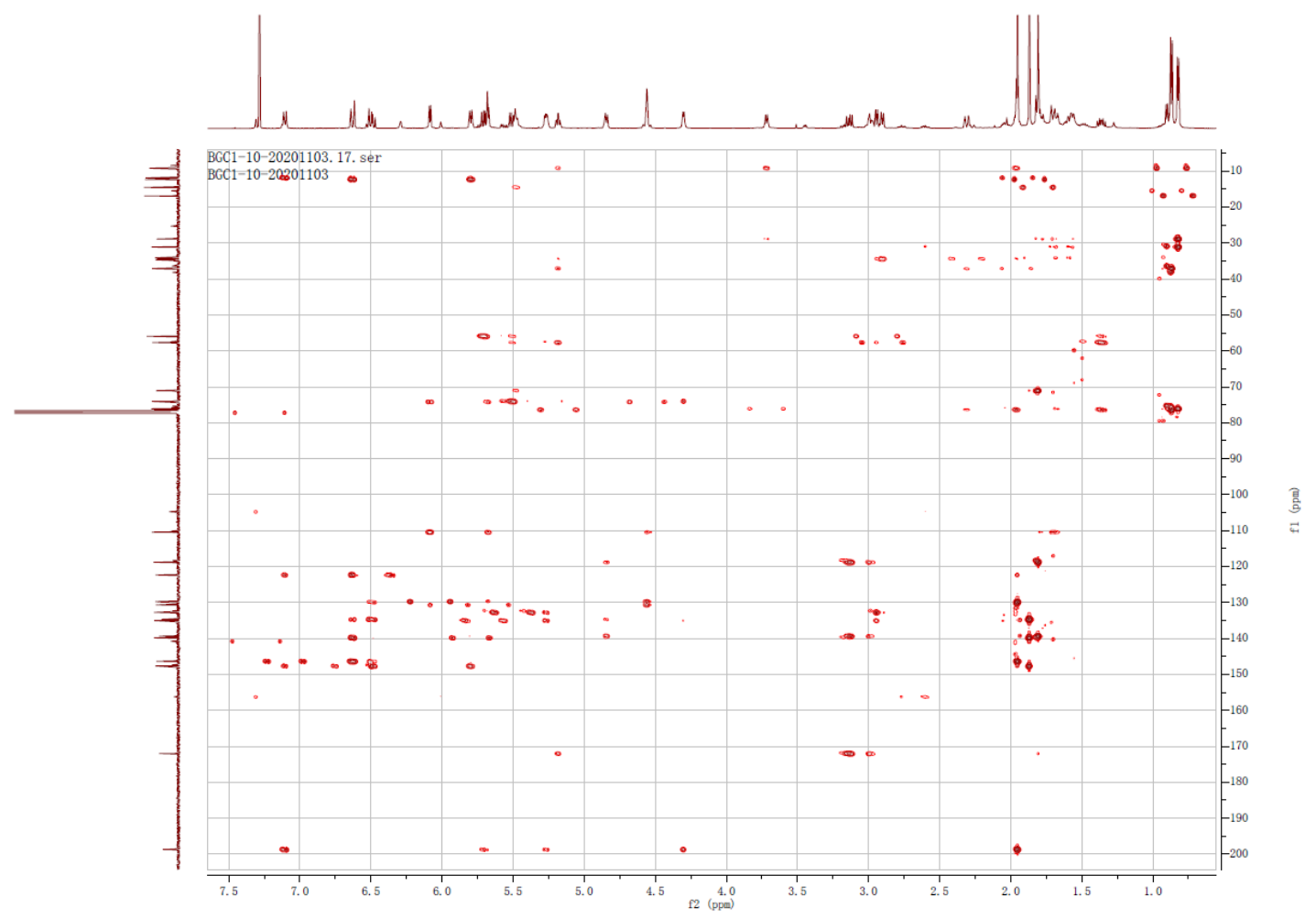

Figure S14. $\mathrm{HMBC}$ spectrum $(150 \mathrm{MHz} / 600 \mathrm{MHz})$ of shuangdaolide $\mathrm{A}$ (1) in $\mathrm{CDCl}_{3}$. 


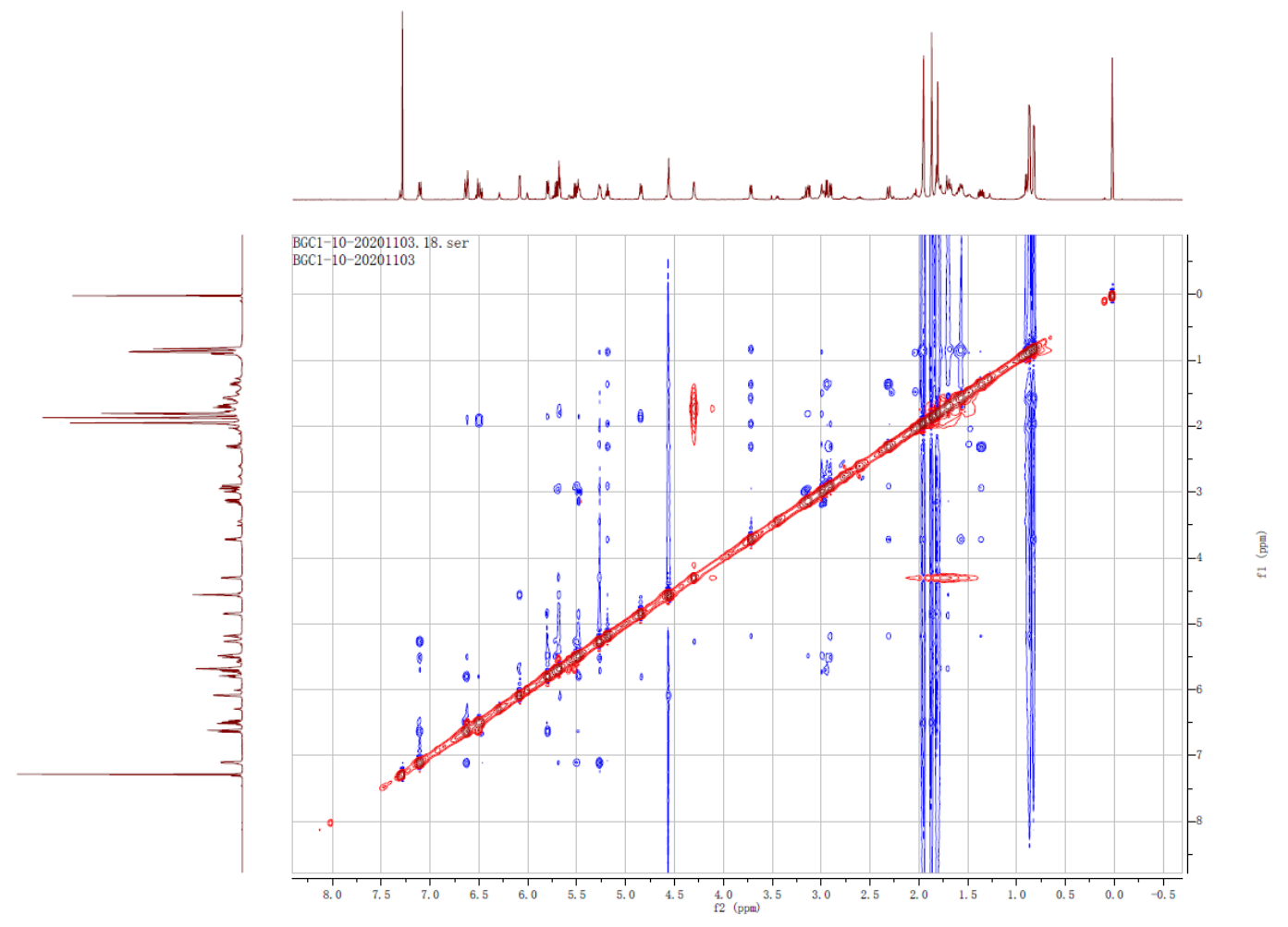

Figure S15. NOESY spectrum $(600 \mathrm{MHz})$ of shuangdaolide $\mathrm{A}(\mathbf{1})$ in $\mathrm{CDCl}_{3}$.

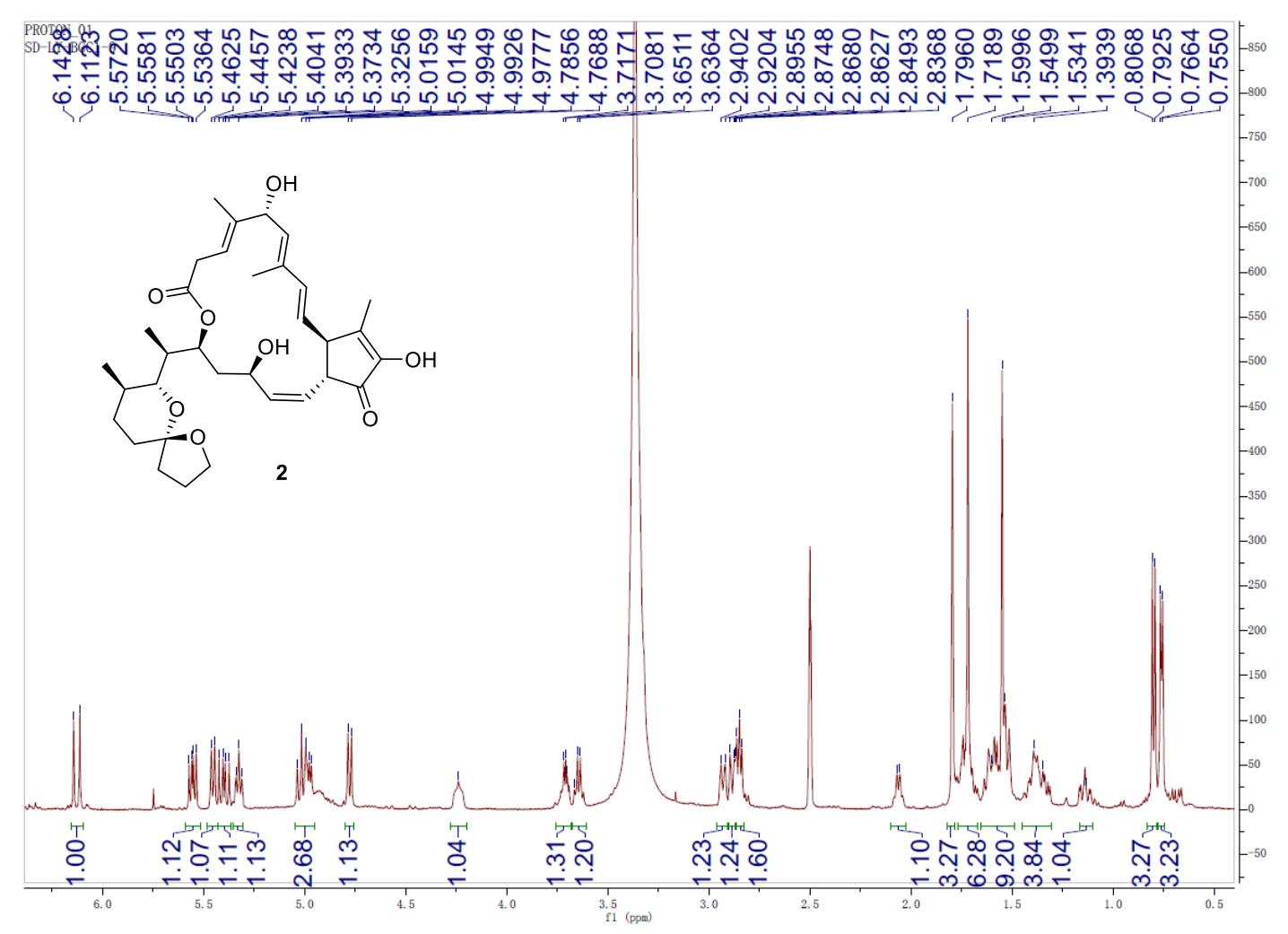

Figure S16. ${ }^{1} \mathrm{H}$ NMR spectrum $(500 \mathrm{MHz})$ of shuangdaolide B (2) in DMSO- $d_{6}$. 


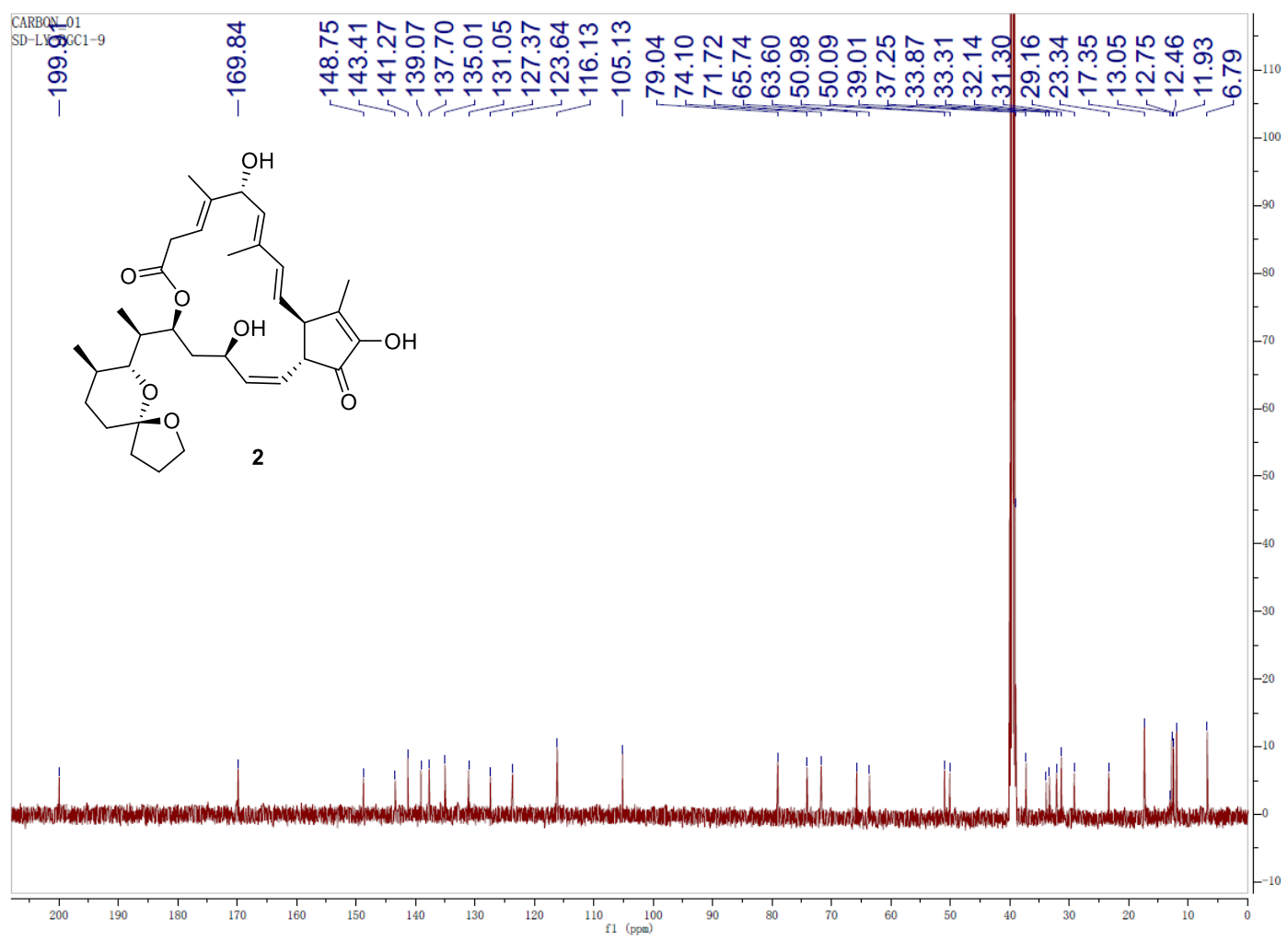

Figure S17. ${ }^{13} \mathrm{C}$ NMR spectrum $(125 \mathrm{MHz})$ of shuangdaolide B (2) in DMSO- $d_{6}$.

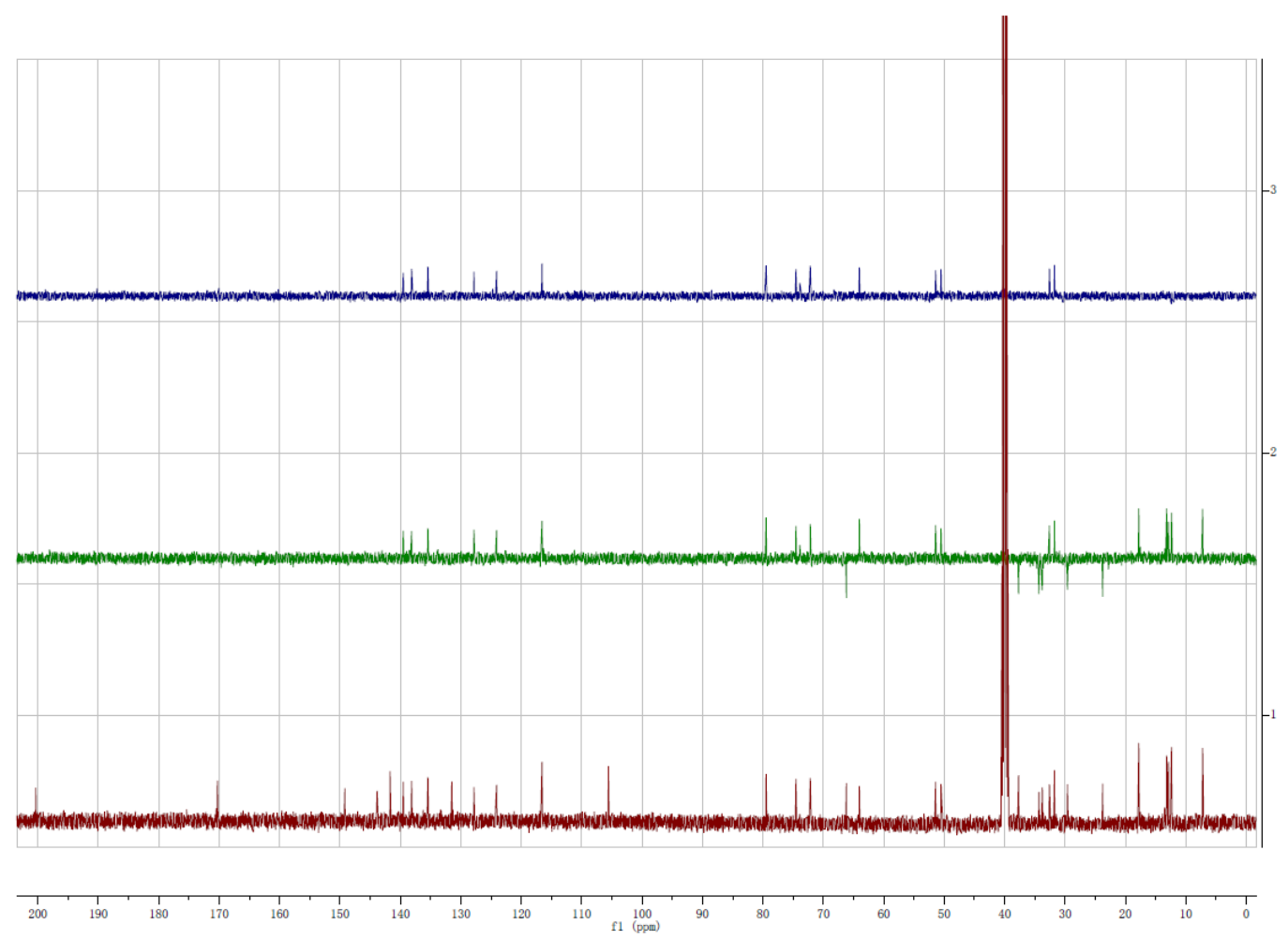

Figure S18. DEPT spectrum (125 MHz) of shuangdaolide B (2) in DMSO- $d_{6}$. 


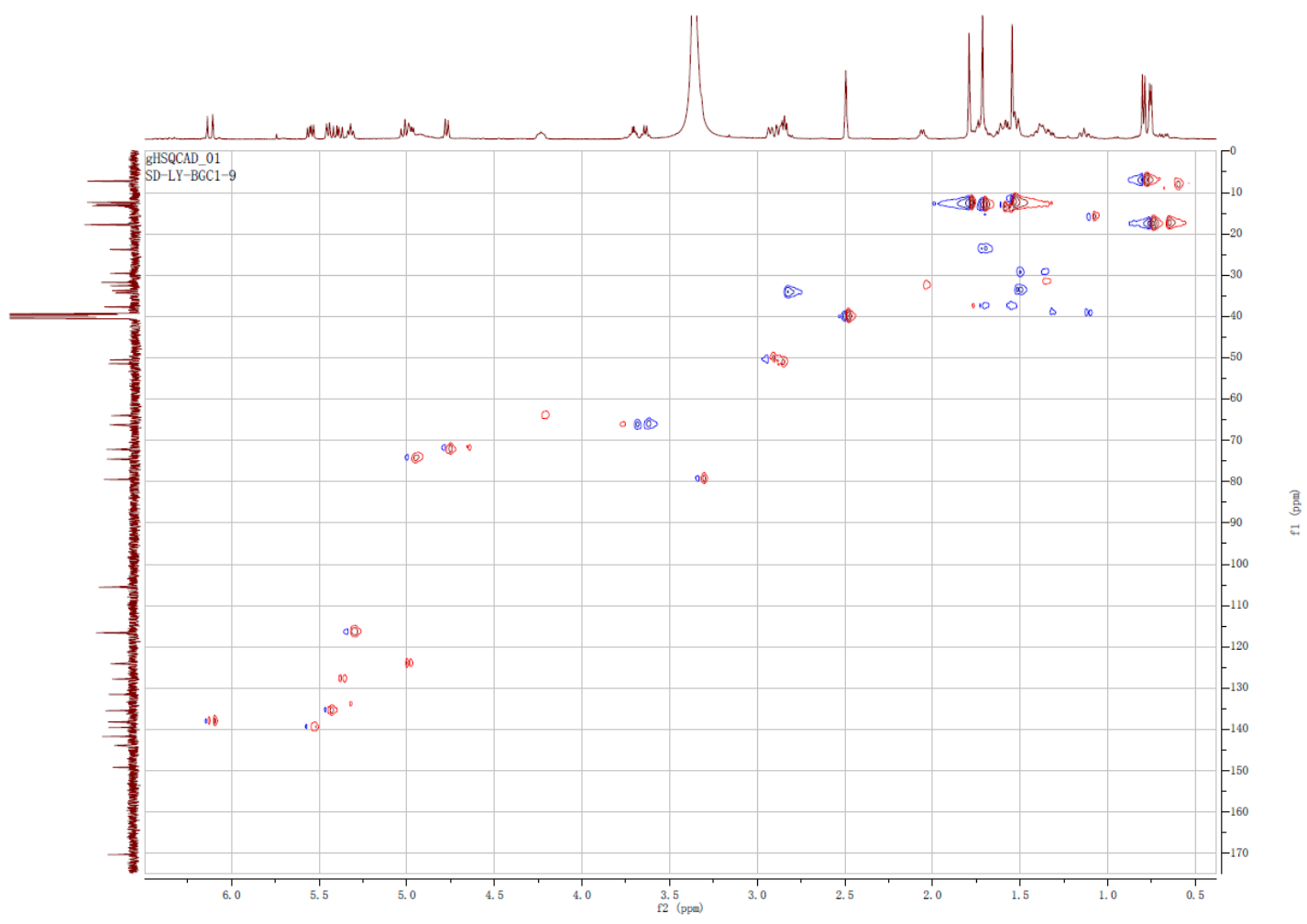

Figure S19. HSQC spectrum (125 MHz/500 MHz) of shuangdaolide B (2) in DMSO- $d_{6}$.

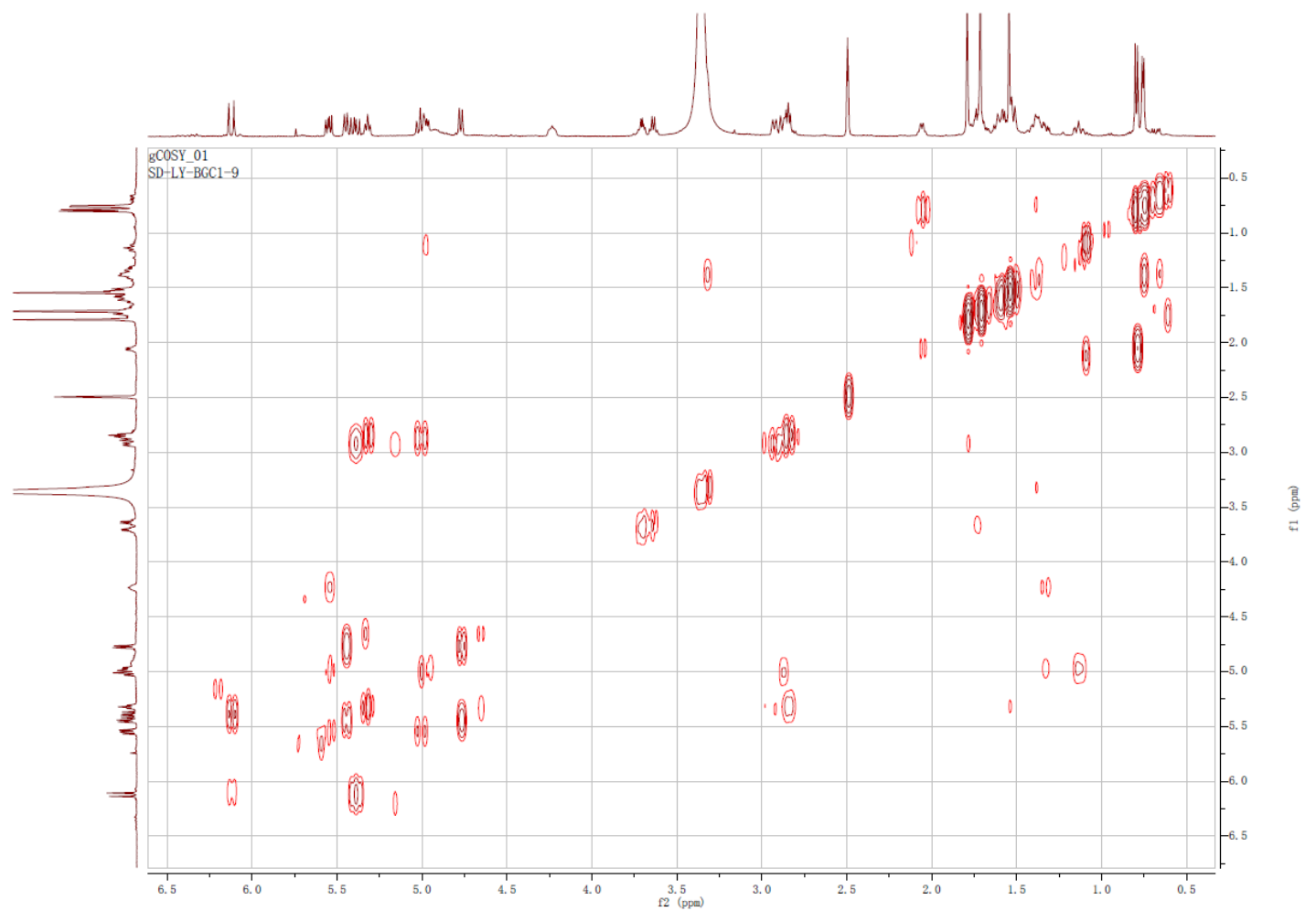

Figure S20. ${ }^{1} \mathrm{H}-{ }^{1} \mathrm{H}$ COSY spectrum $(500 \mathrm{MHz})$ of shuangdaolide B (2) in DMSO- $d_{6}$. 


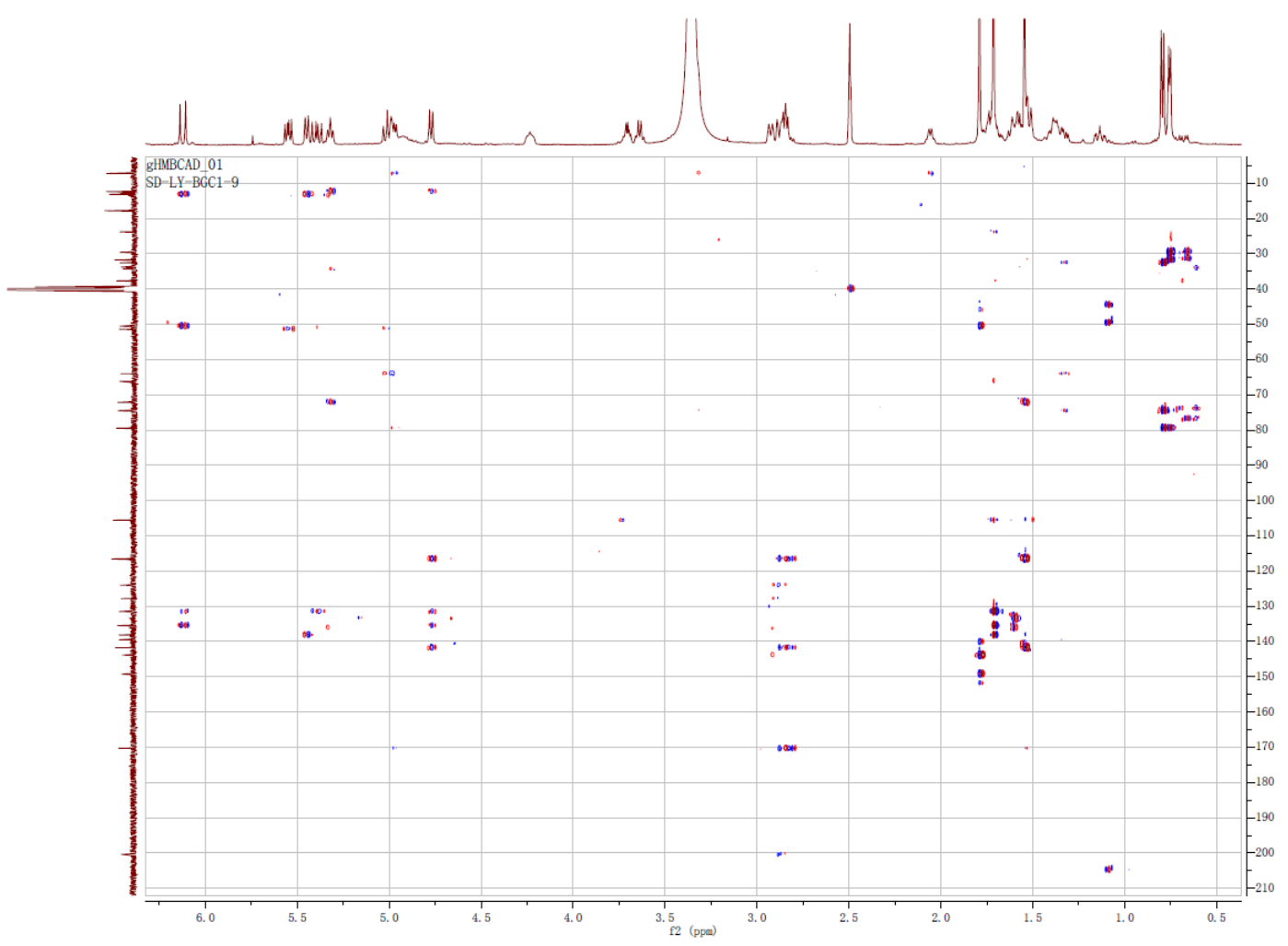

Figure S21. HMBC spectrum (125 MHz/500 MHz) of shuangdaolide B (2) in DMSO- $d_{6}$.

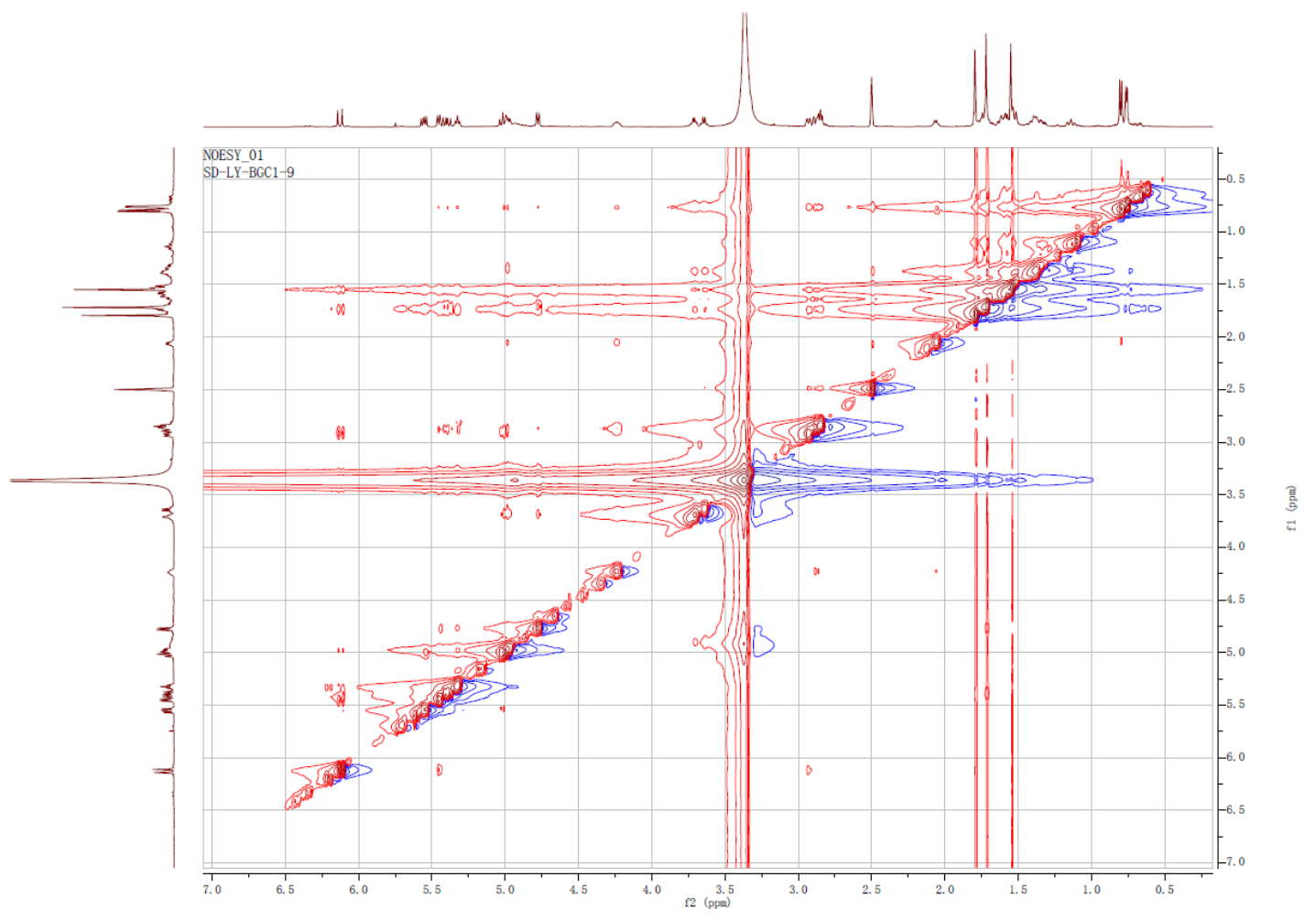

Figure S22. NOESY spectrum $(500 \mathrm{MHz})$ of shuangdaolide B (2) in DMSO- $d_{6}$. 


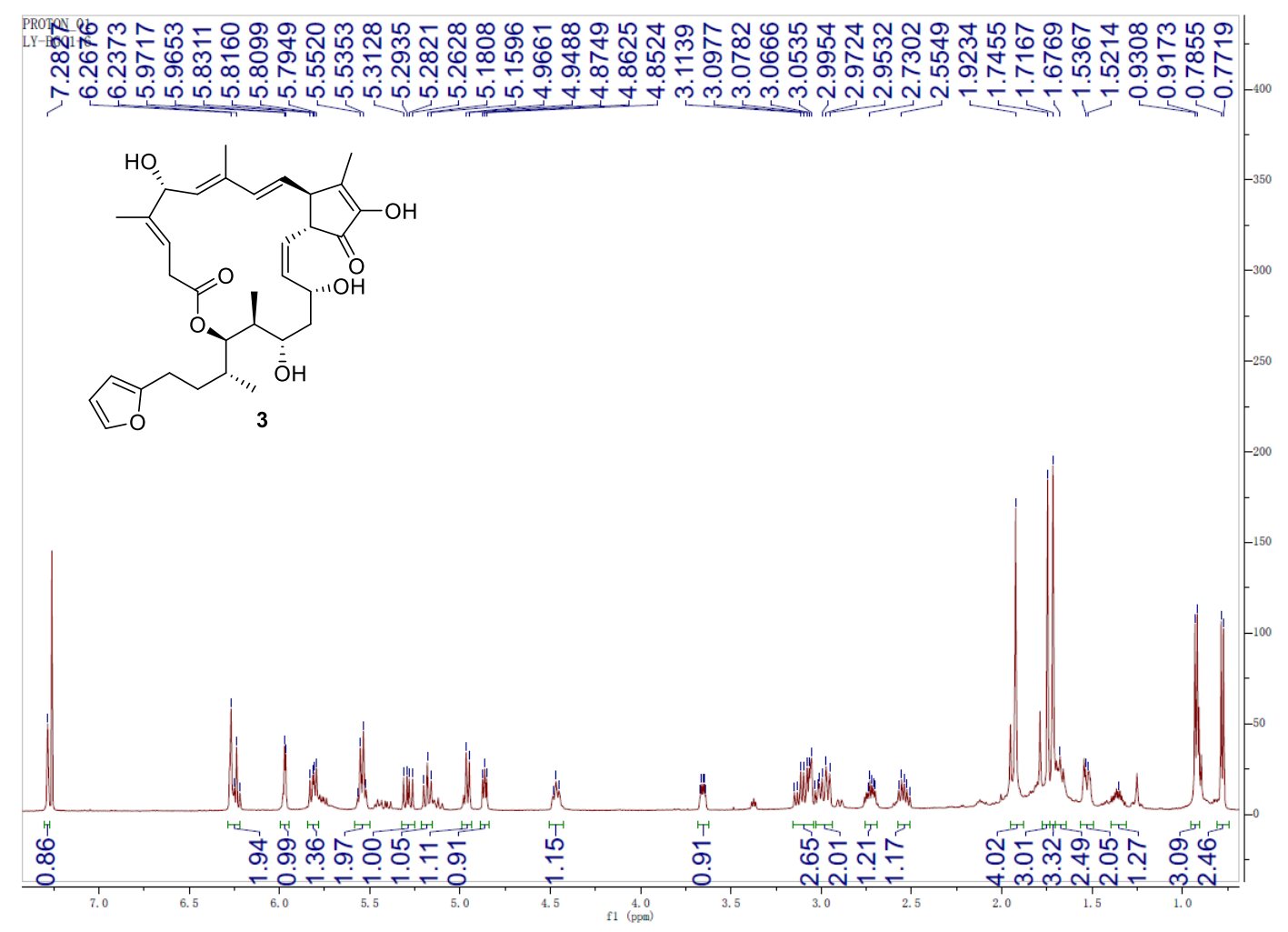

Figure S23. ${ }^{1} \mathrm{H}$ NMR spectrum $(600 \mathrm{MHz})$ of shuangdaolide $\mathrm{C}(\mathbf{3})$ in $\mathrm{CDCl}_{3}$.

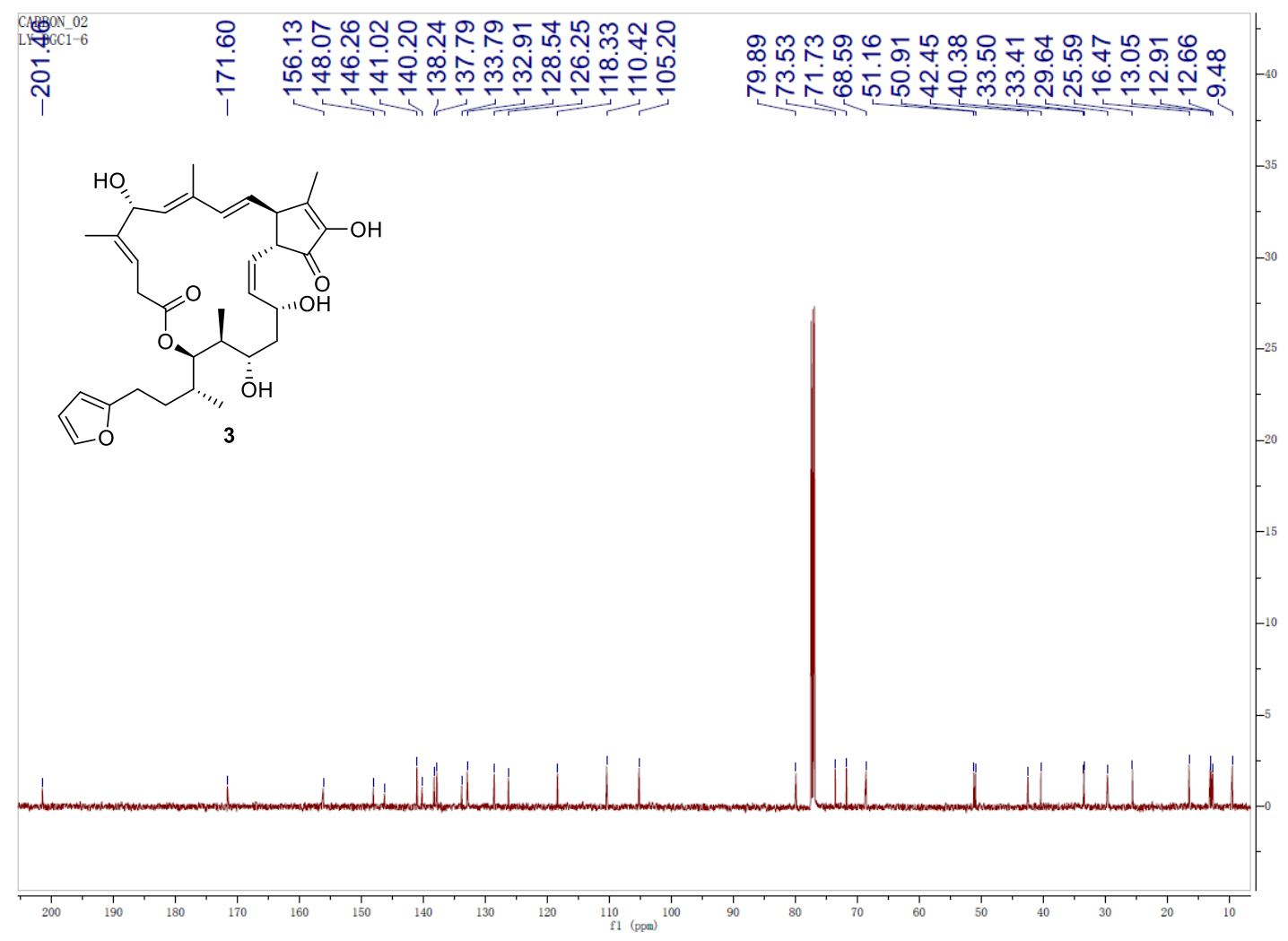

Figure S24. ${ }^{13} \mathrm{C}$ NMR spectrum $(150 \mathrm{MHz})$ of shuangdaolide $\mathrm{C}(3)$ in $\mathrm{CDCl}_{3}$. 

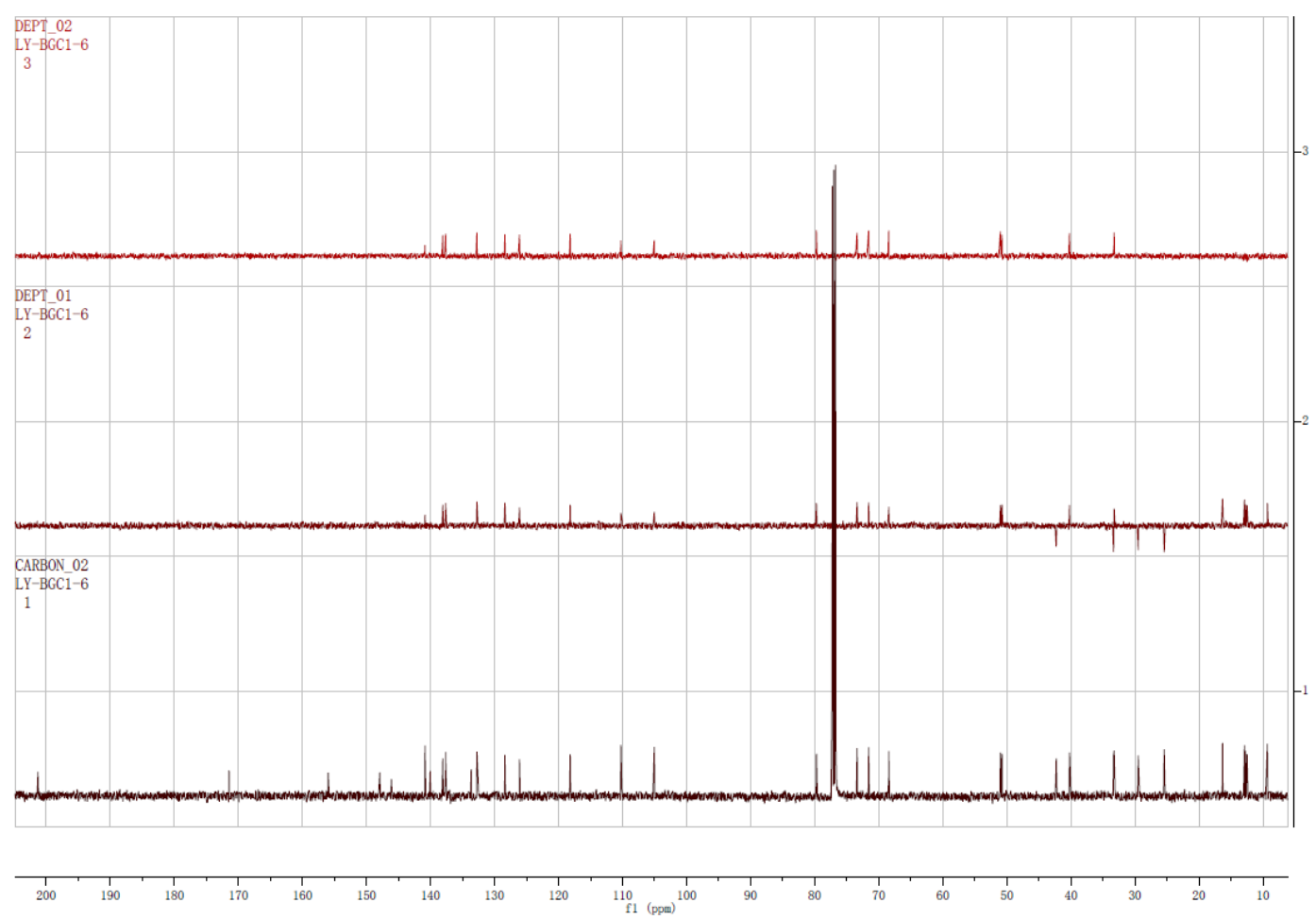

Figure S25. DEPT spectrum (150 MHz) of shuangdaolide C (3) in $\mathrm{CDCl}_{3}$.

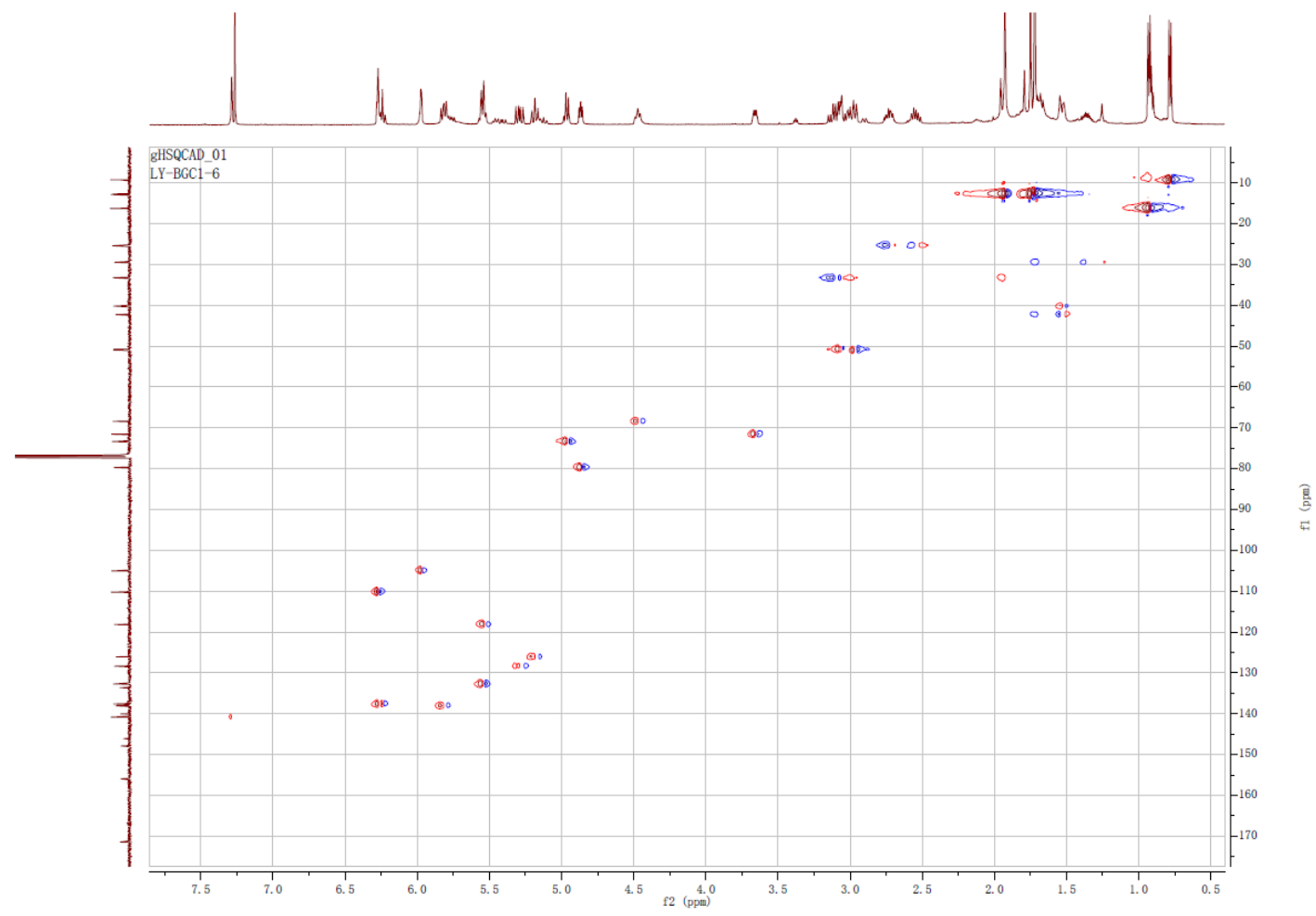

Figure S26. HSQC spectrum (150 MHz/600 MHz) of shuangdaolide C (3) in $\mathrm{CDCl}_{3}$. 


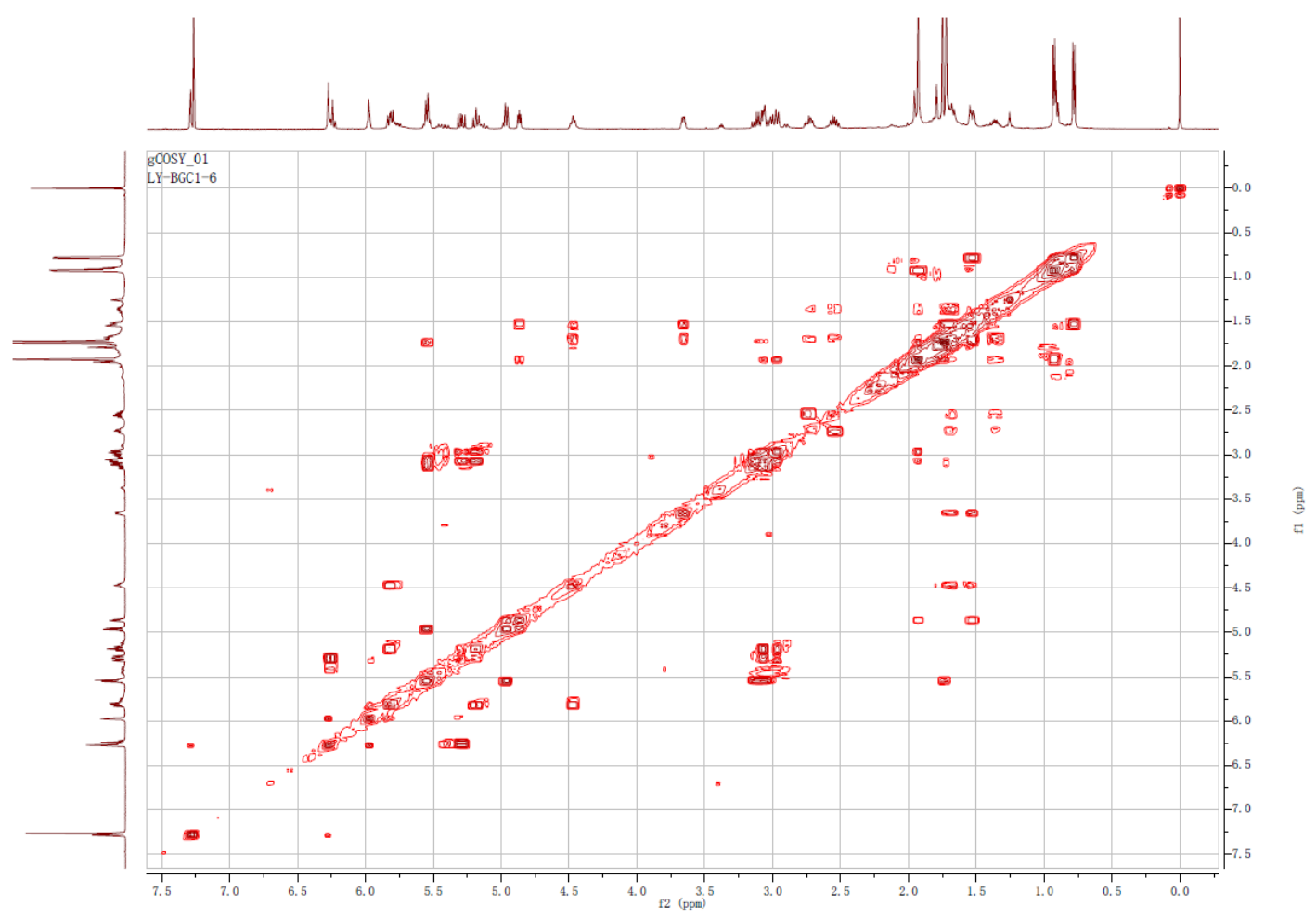

Figure S27. ${ }^{1} \mathrm{H}-{ }^{1} \mathrm{H}$ COSY spectrum $(600 \mathrm{MHz})$ of shuangdaolide $\mathrm{C}(3)$ in $\mathrm{CDCl}_{3}$.

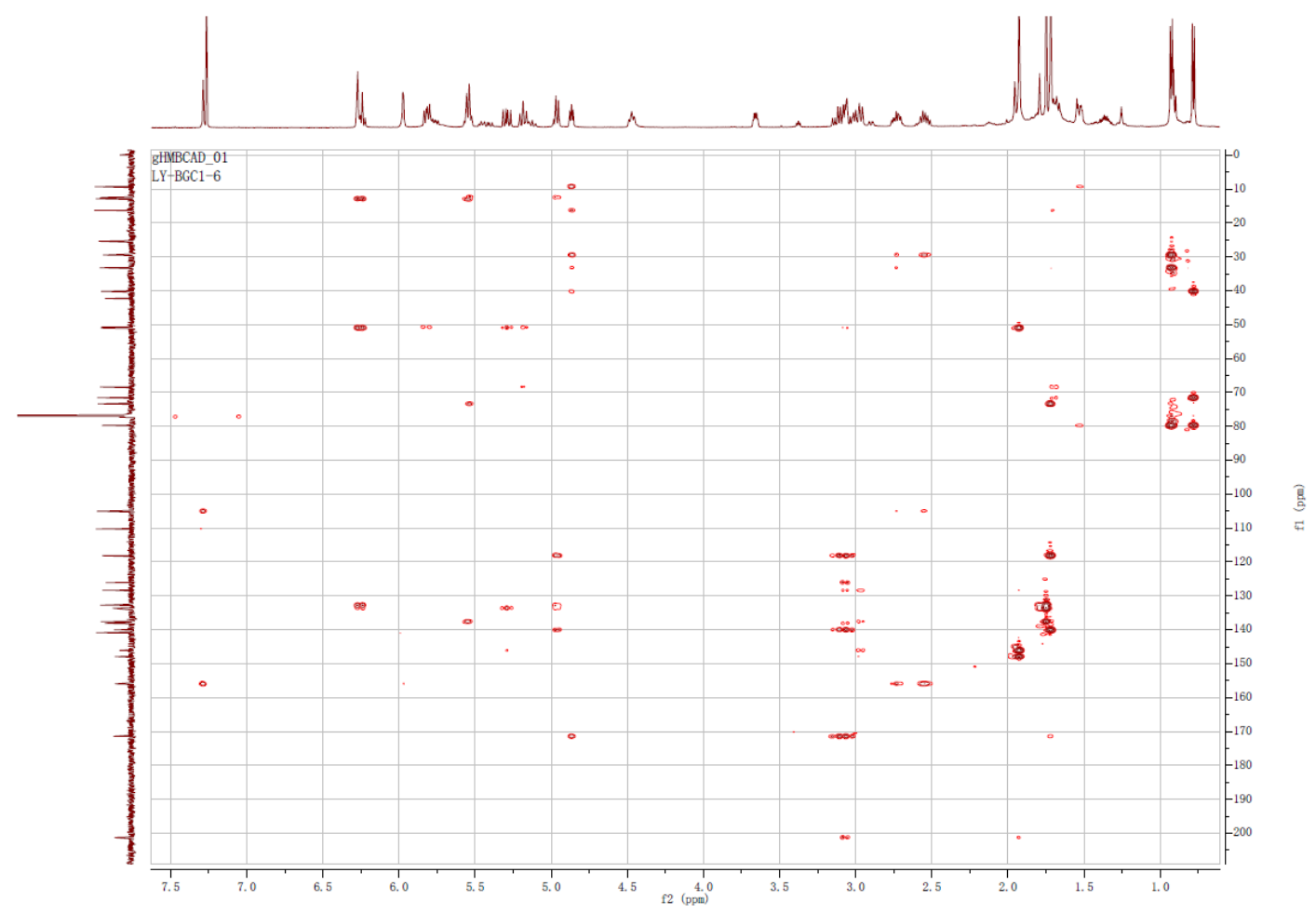

Figure S28. HMBC spectrum (150 MHz/600 MHz) of shuangdaolide C (3) in $\mathrm{CDCl}_{3}$. 


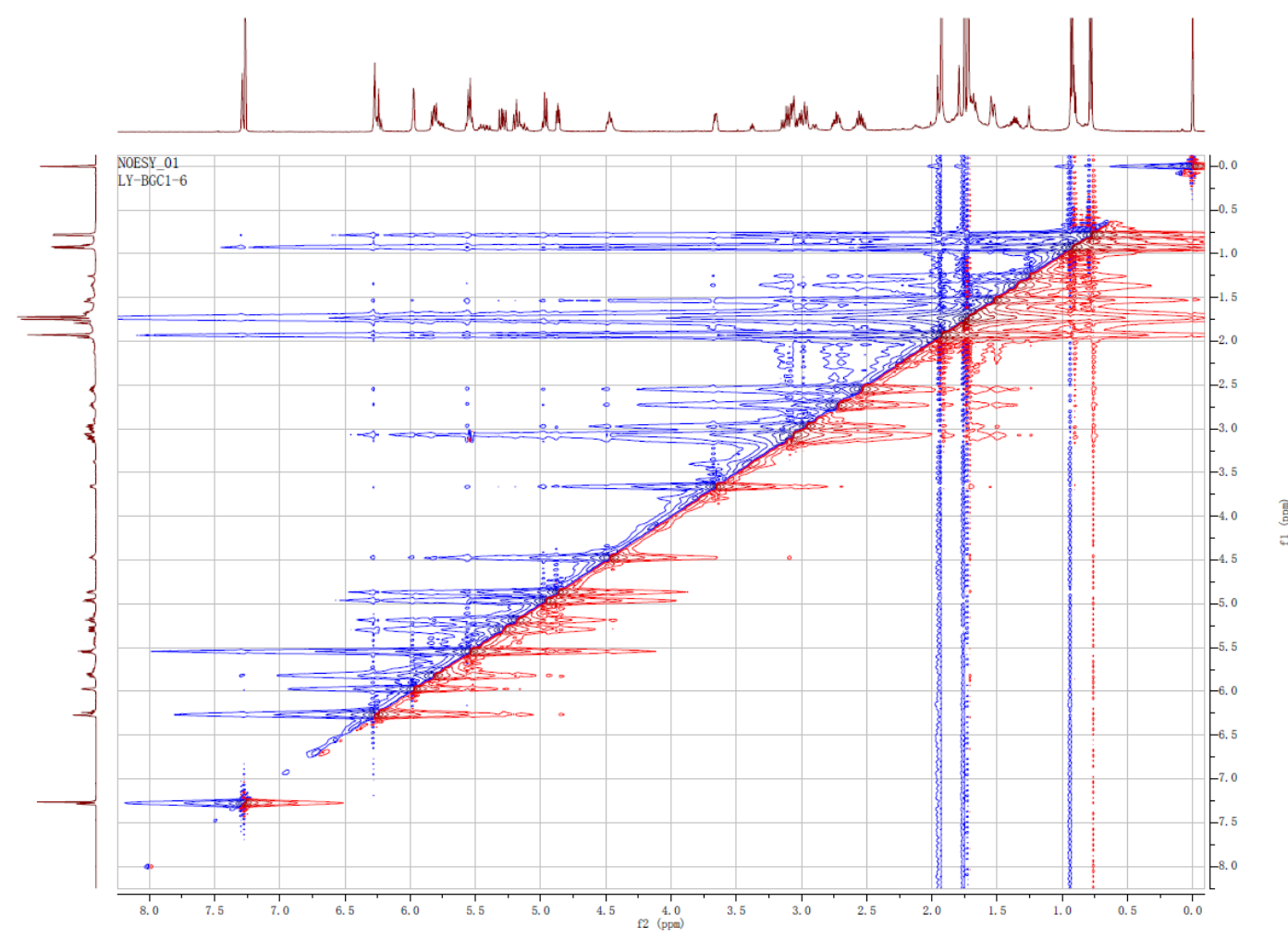

Figure S29. NOESY spectrum $(600 \mathrm{MHz})$ of shuangdaolide $\mathrm{C} \mathrm{(3)}$ in $\mathrm{CDCl}_{3}$.

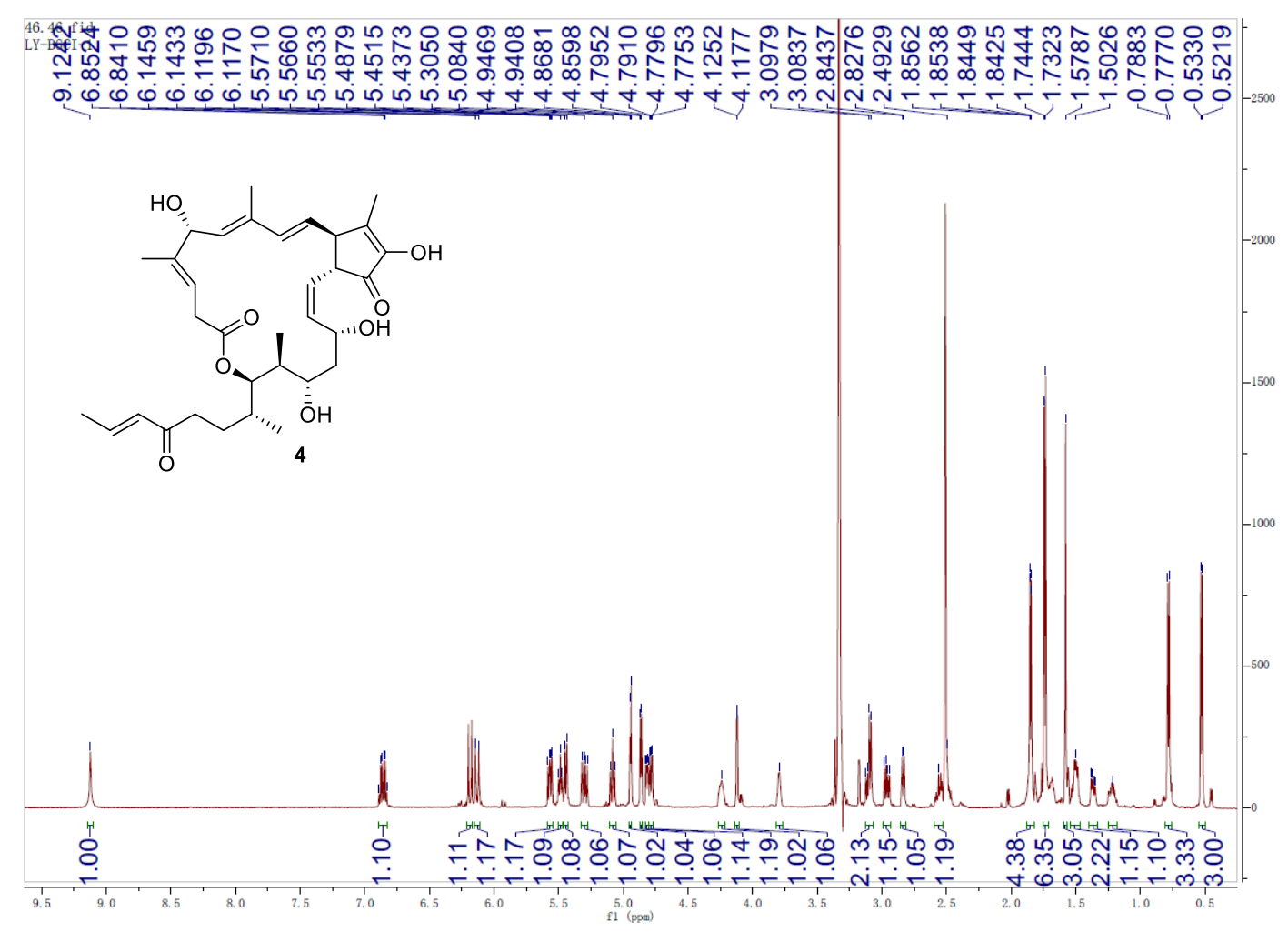

Figure S30. ${ }^{1} \mathrm{H}$ NMR spectrum $(600 \mathrm{MHz})$ of shuangdaolide D (4) in DMSO- $d_{6}$. 


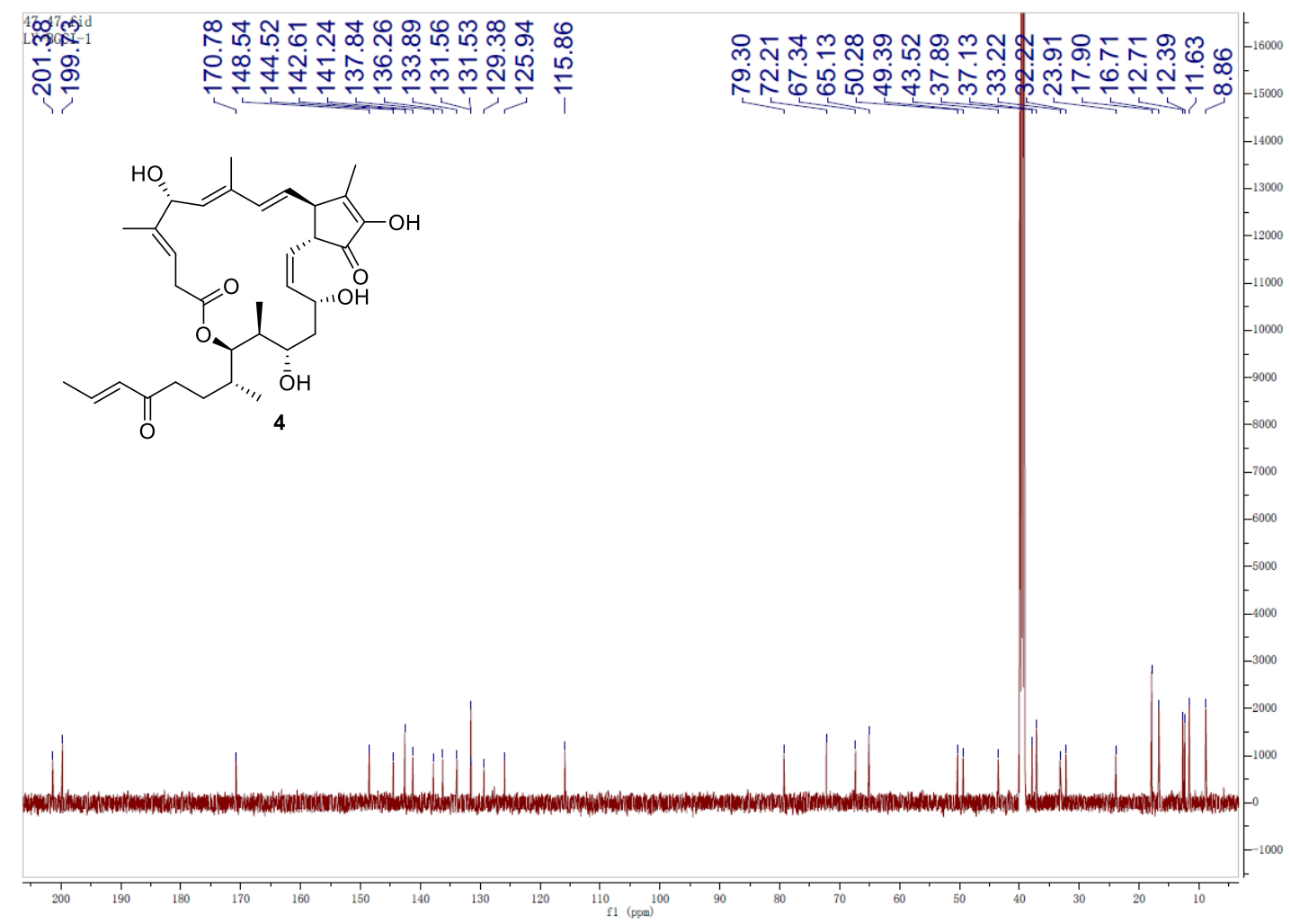

Figure S31. ${ }^{13} \mathrm{C}$ NMR spectrum (150 MHz) of shuangdaolide D (4) in DMSO- $d_{6}$.
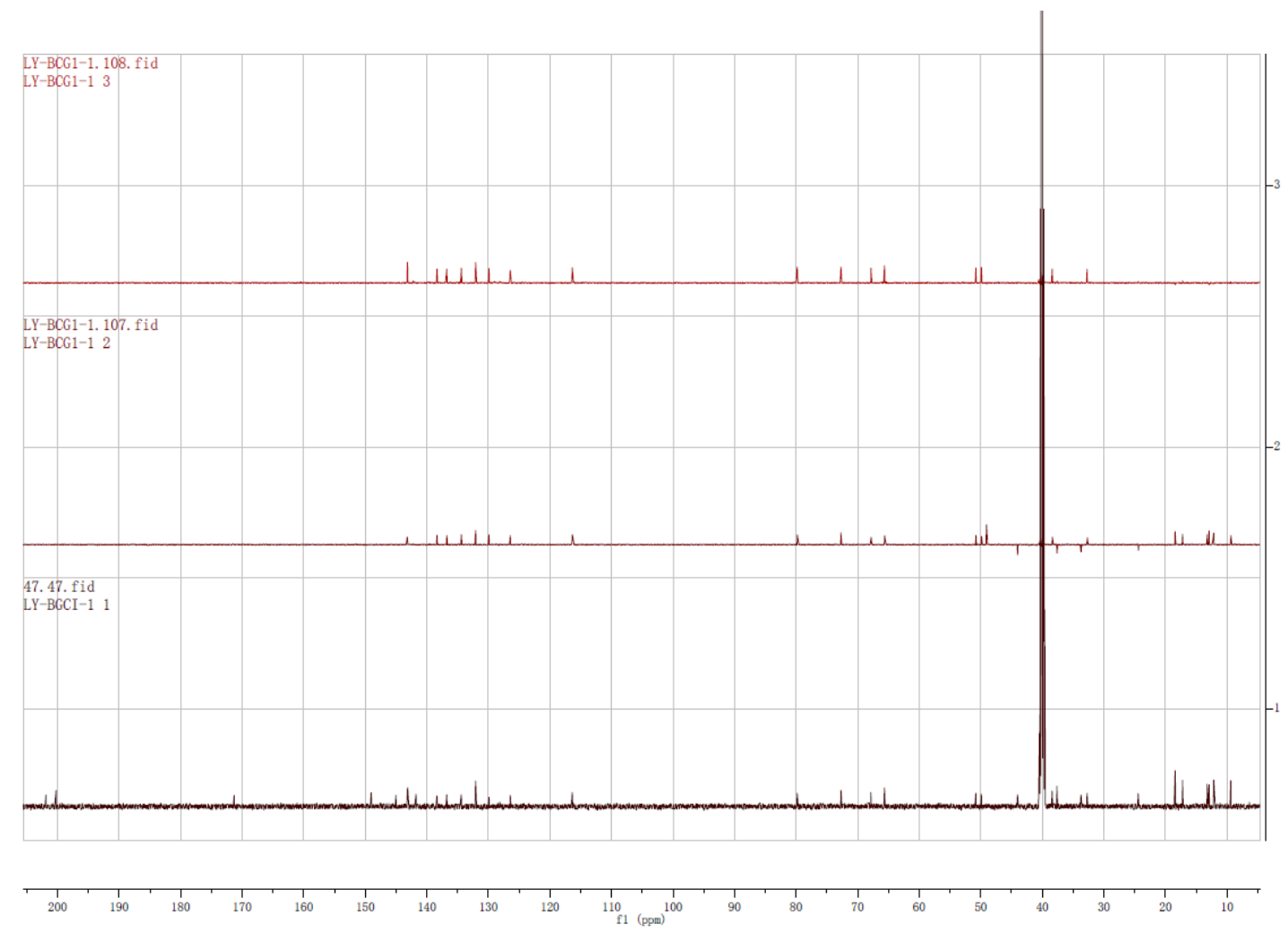

Figure S32. DEPT spectrum (150 MHz) of shuangdaolide D (4) in DMSO- $d_{6}$. 


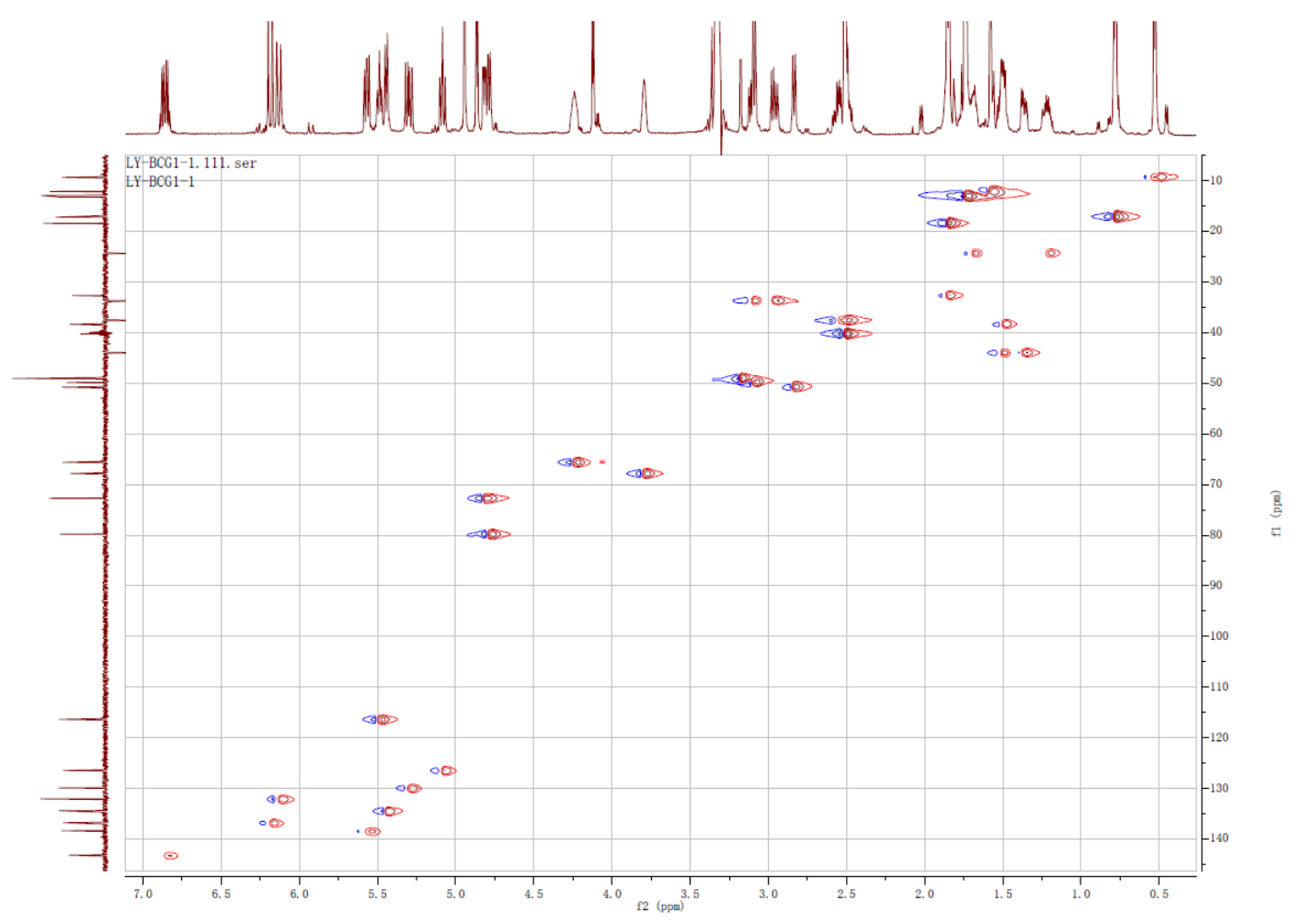

Figure S33. HSQC spectrum (150 MHz/600 MHz) of shuangdaolide D (4) in DMSO- $d_{6}$.

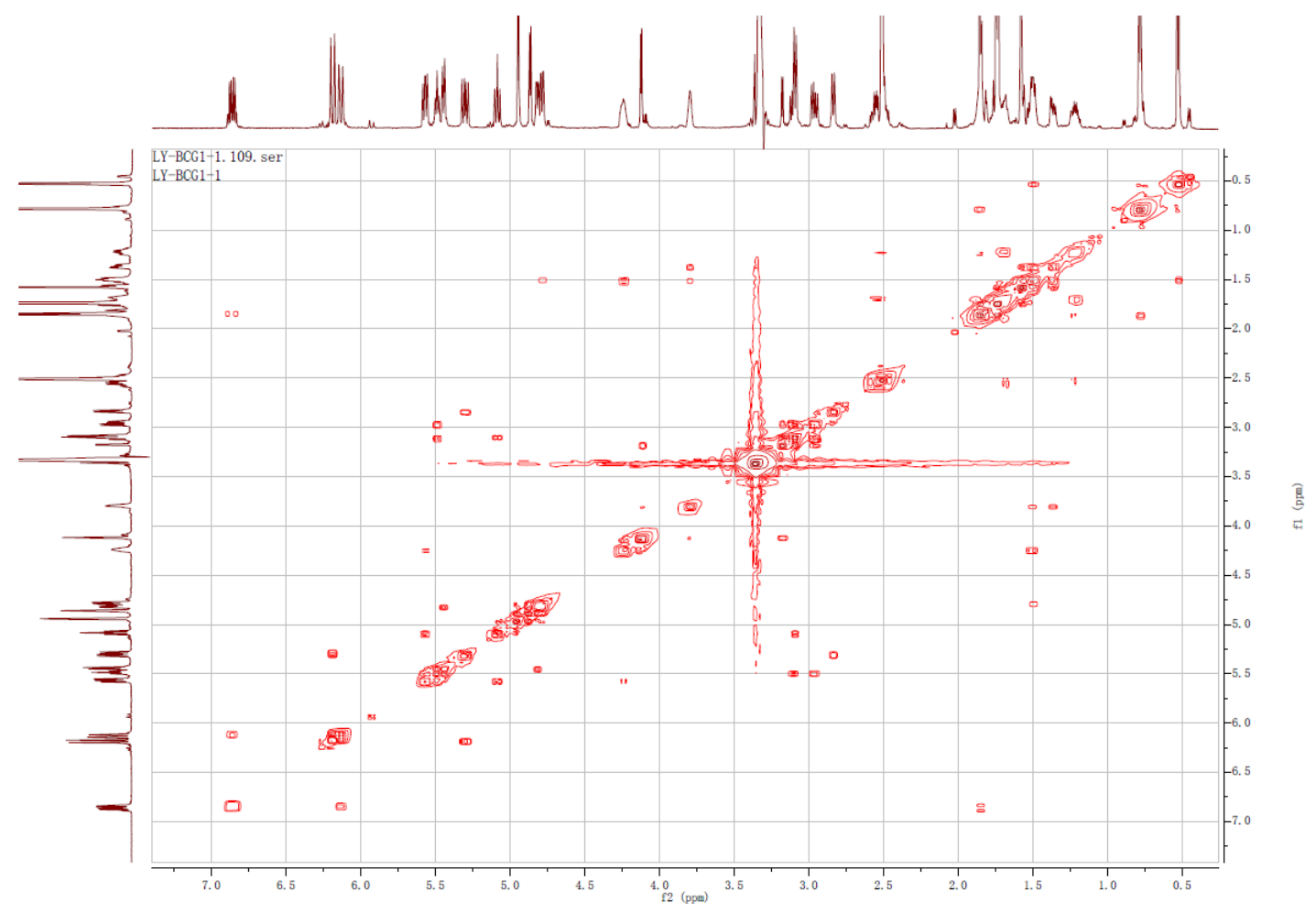

Figure S34. ${ }^{1} \mathrm{H}-{ }^{1} \mathrm{H}$ COSY spectrum $(600 \mathrm{MHz})$ of shuangdaolide D (4) in DMSO- $d_{6}$. 


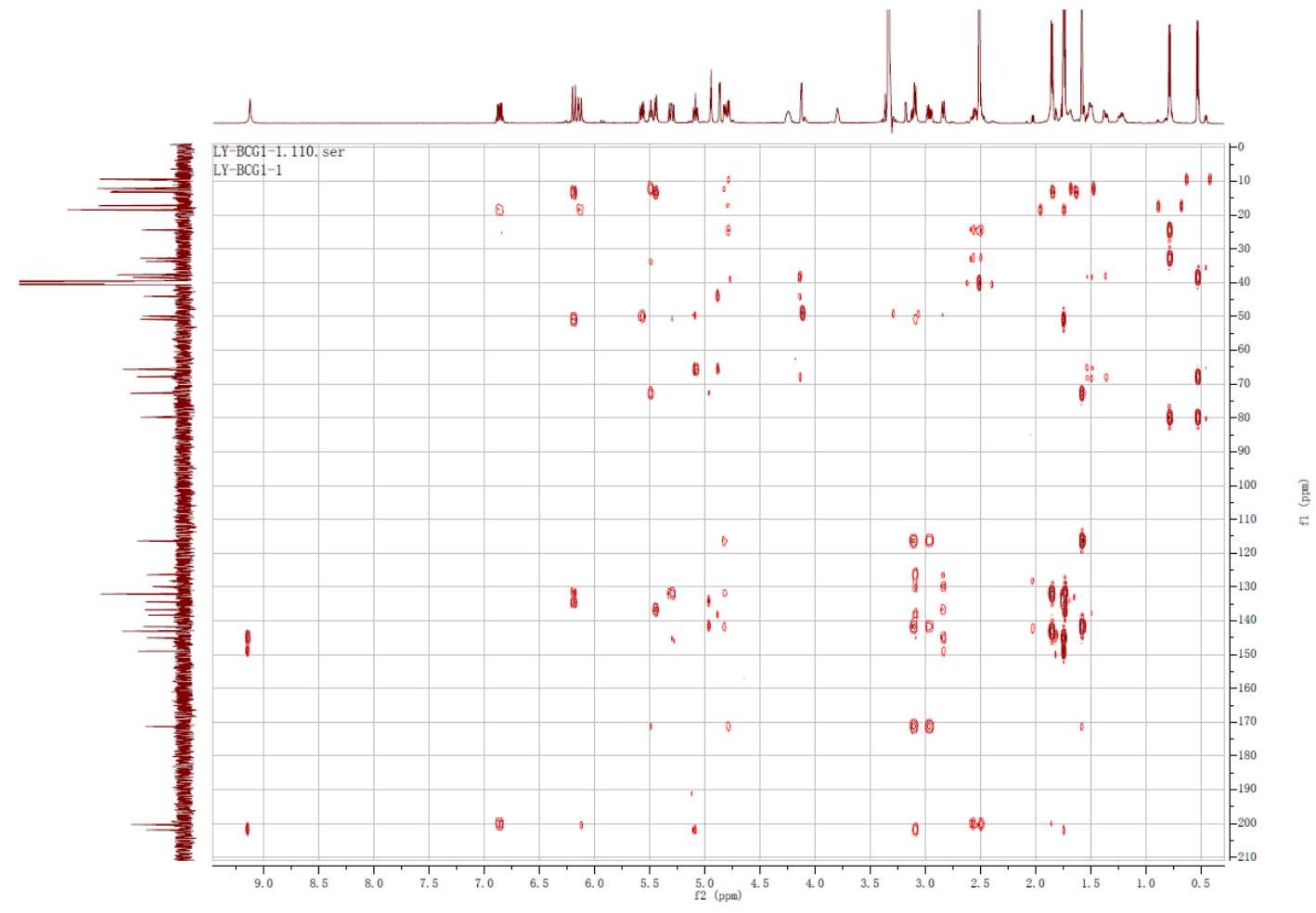

Figure S35. HMBC spectrum (150 MHz/600 MHz) of shuangdaolide D (4) in DMSO- $d_{6}$.

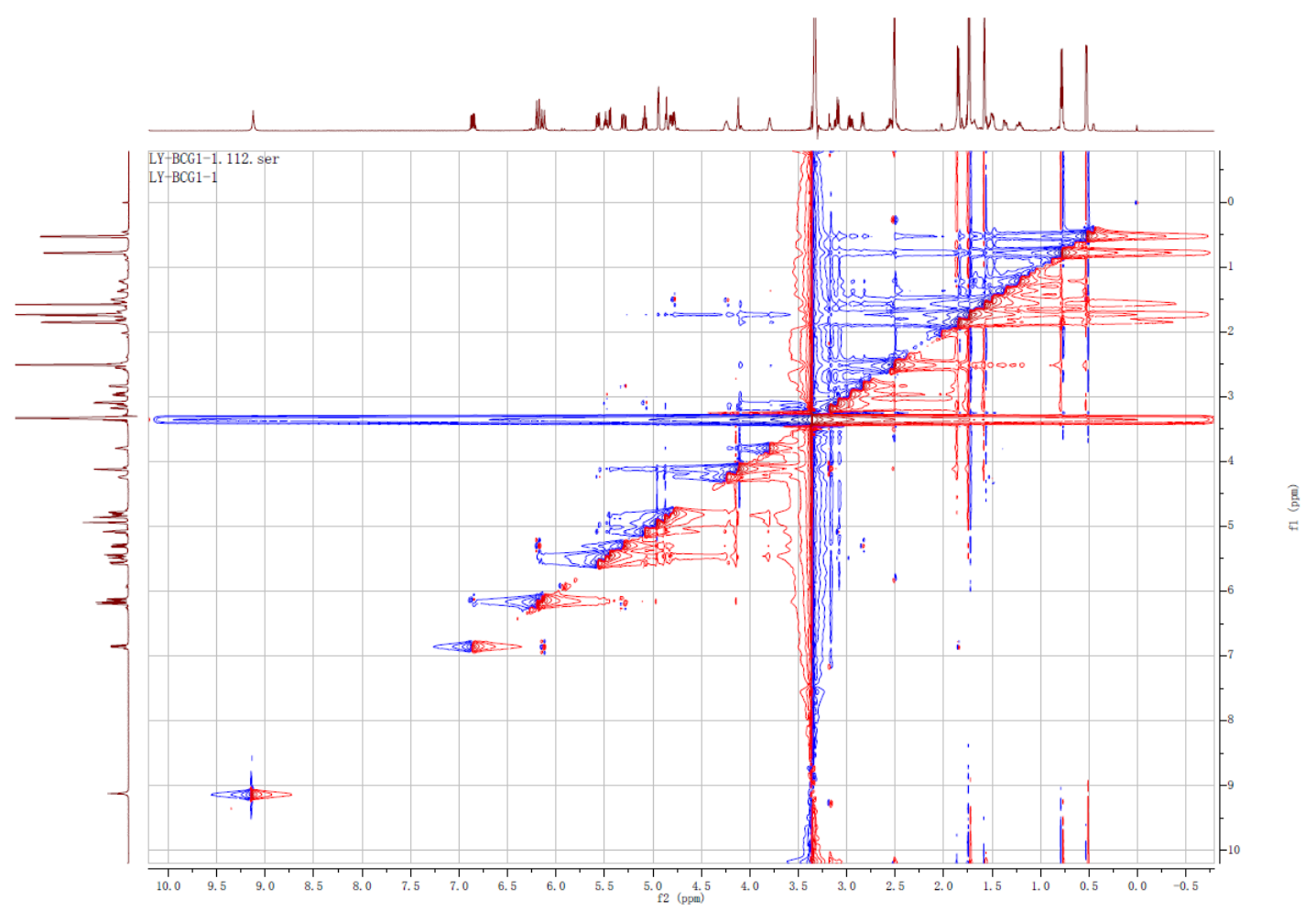

Figure S36. NOESY spectrum (600 MHz) of shuangdaolide D (4) in DMSO- $d_{6}$. 


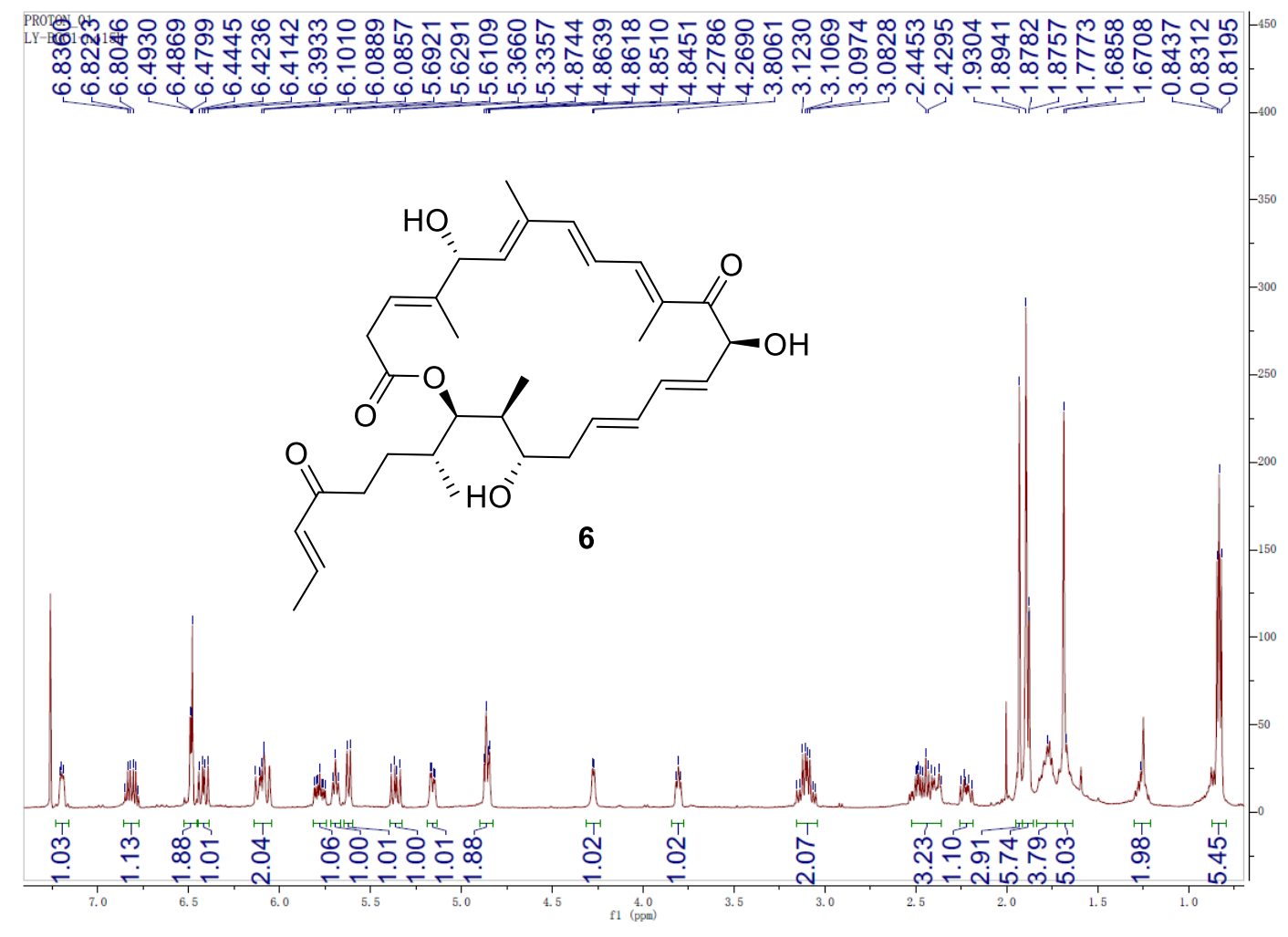

Figure S37. ${ }^{1} \mathrm{H}$ NMR spectrum $(500 \mathrm{MHz})$ of shuangdaolide $\mathrm{F}(6)$ in $\mathrm{CDCl}_{3}$.

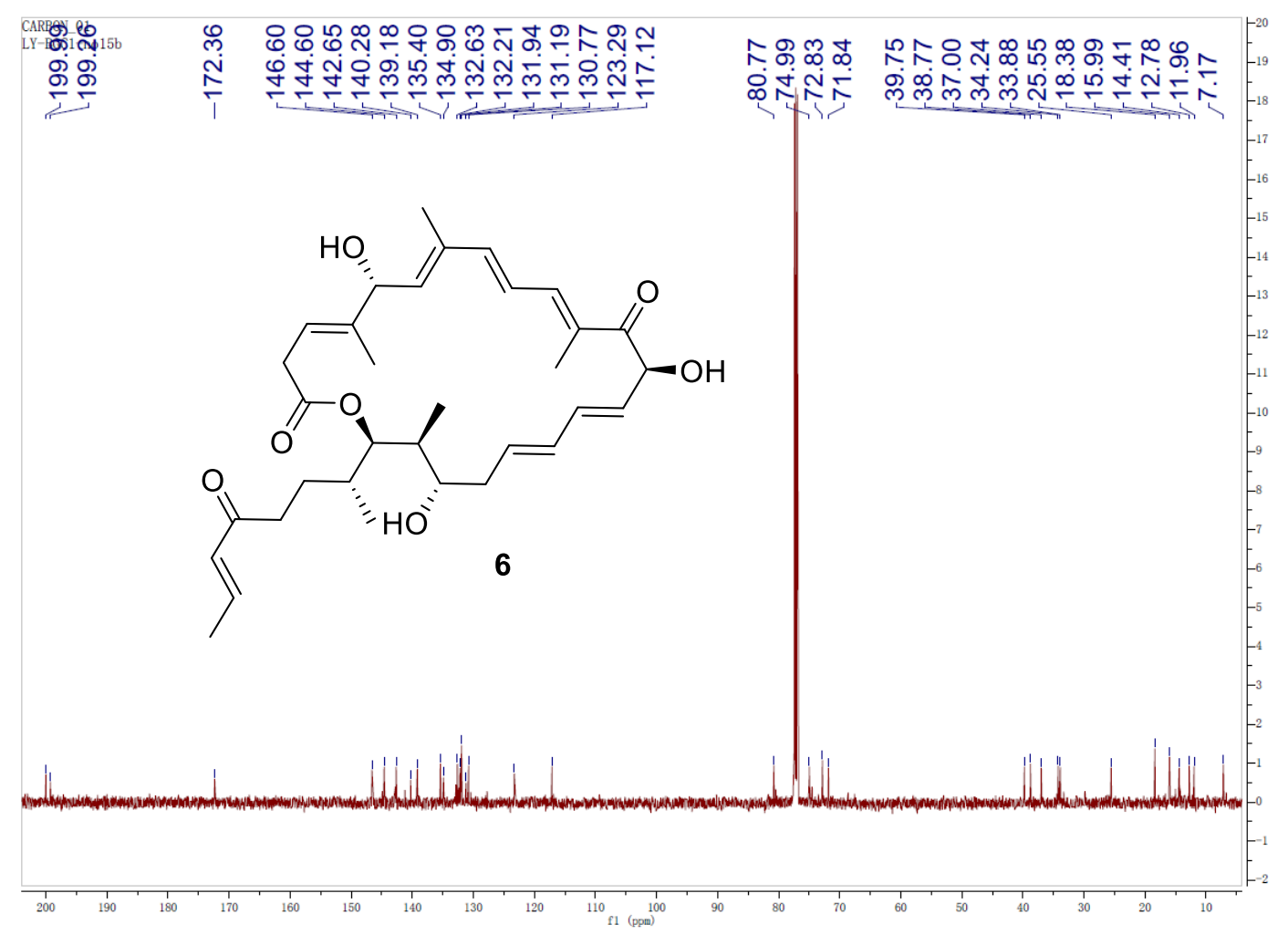

Figure S38. ${ }^{13} \mathrm{C}$ NMR spectrum $(125 \mathrm{MHz})$ of shuangdaolide $\mathrm{F}(6)$ in $\mathrm{CDCl}_{3}$. 

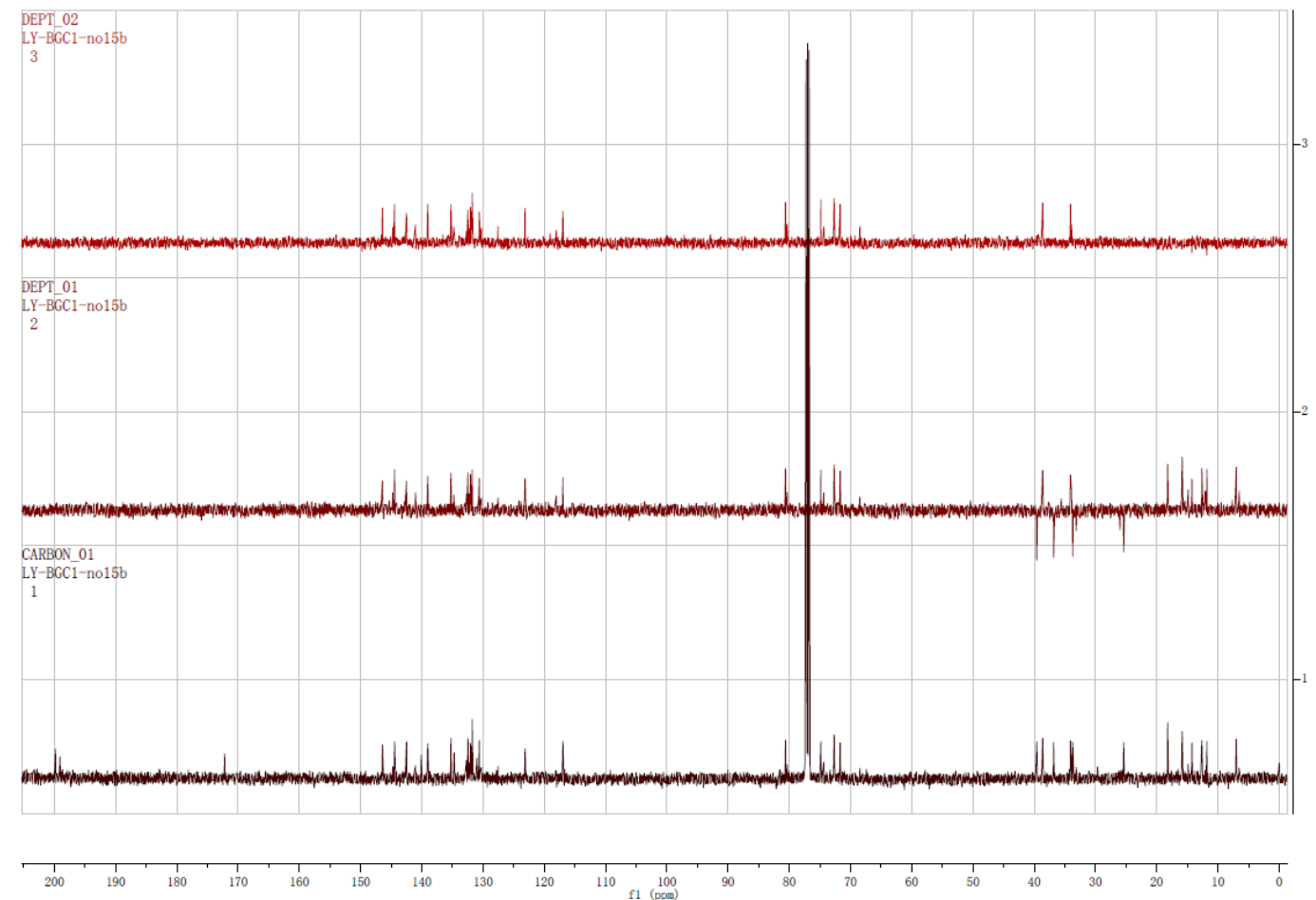

Figure S39. DEPT spectrum (125 MHz) of shuangdaolide $\mathrm{F}(\mathbf{6})$ in $\mathrm{CDCl}_{3}$.

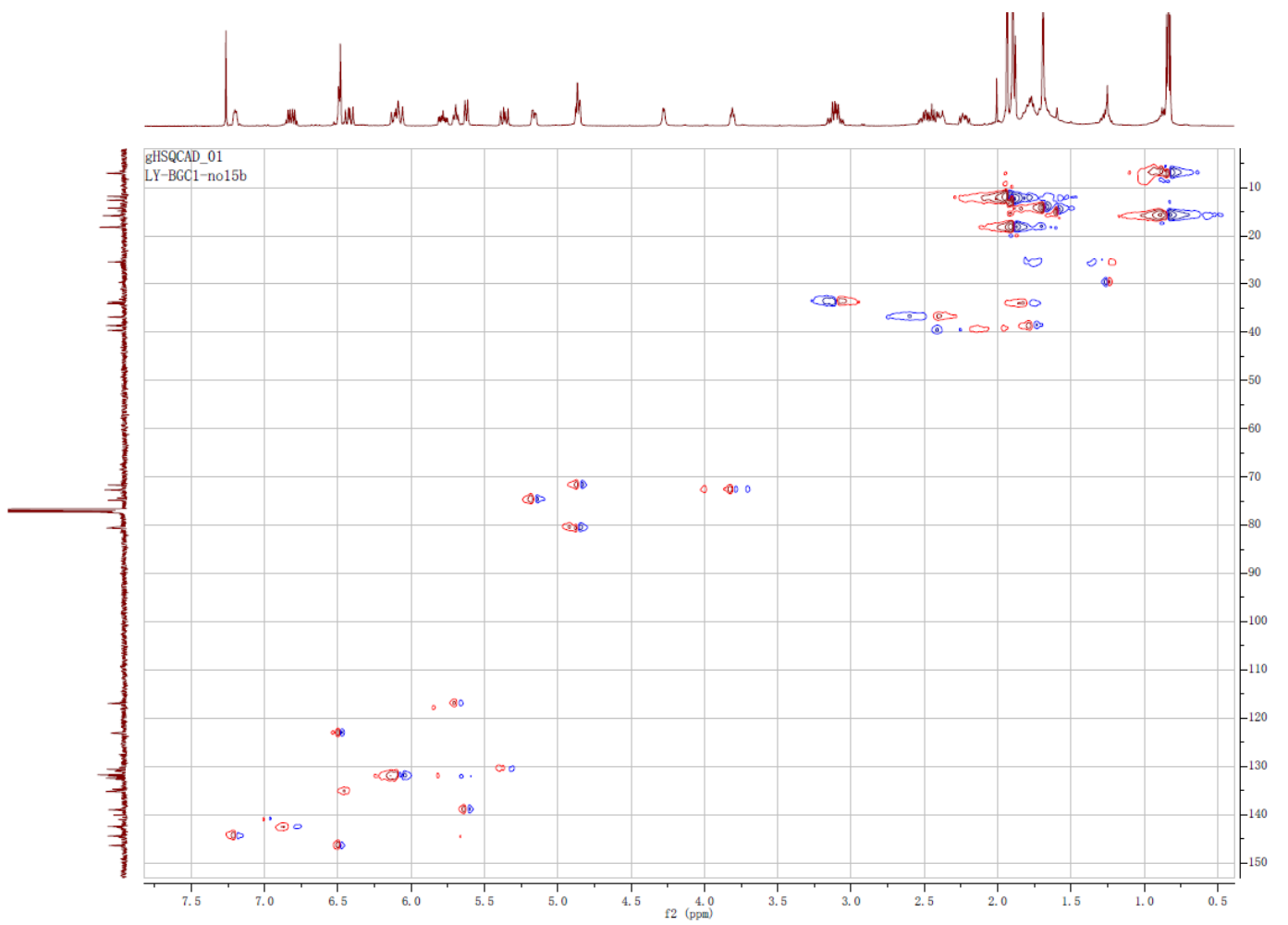

Figure S40. HSQC spectrum (125 MHz/500 MHz) of shuangdaolide $\mathrm{F}(6)$ in $\mathrm{CDCl}_{3}$. 


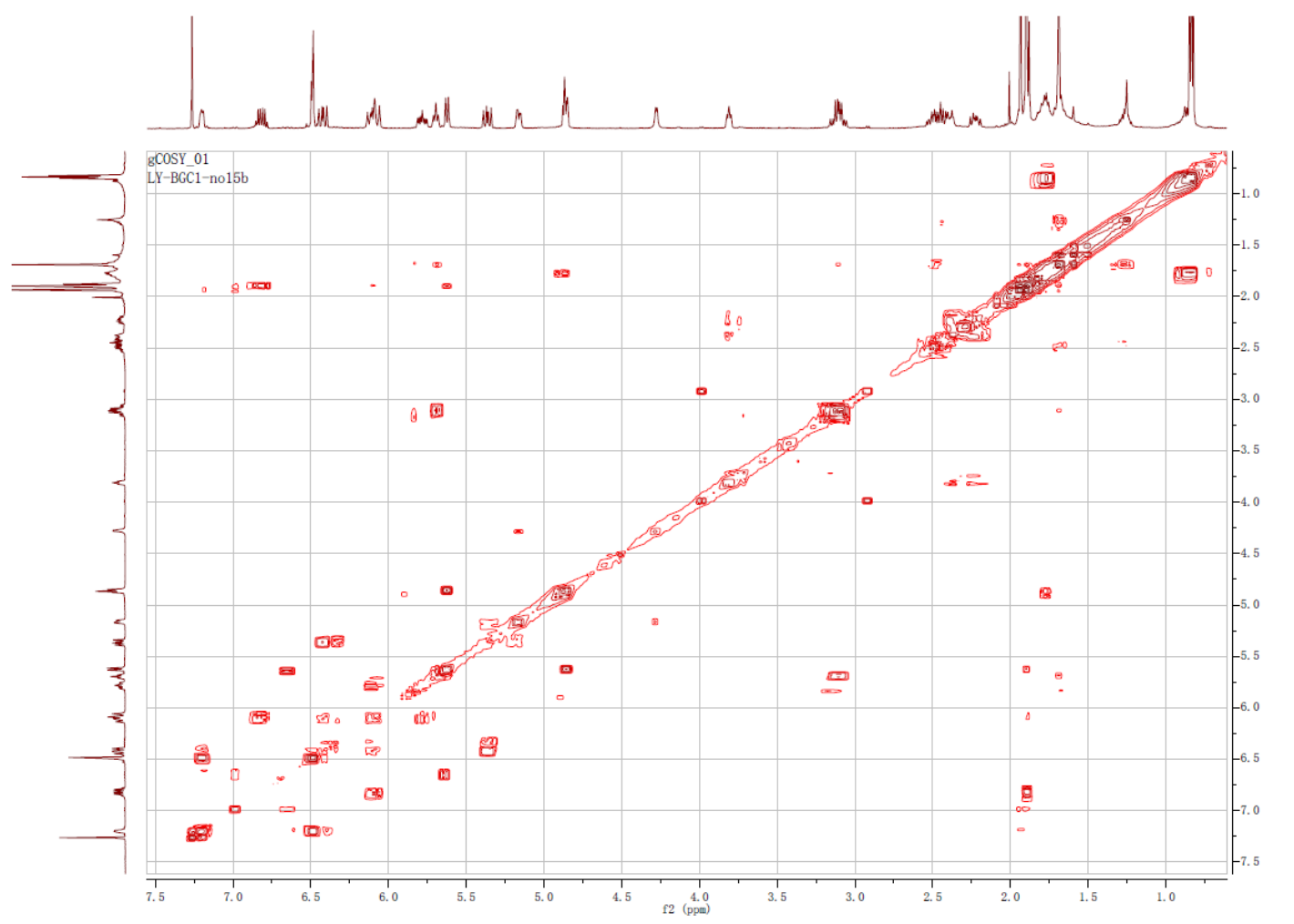

Figure $\mathbf{S 4 1} .{ }^{1} \mathrm{H}-{ }^{-1} \mathrm{H}$ COSY spectrum $(500 \mathrm{MHz})$ of shuangdaolide $\mathrm{F}(6)$ in $\mathrm{CDCl}_{3}$.

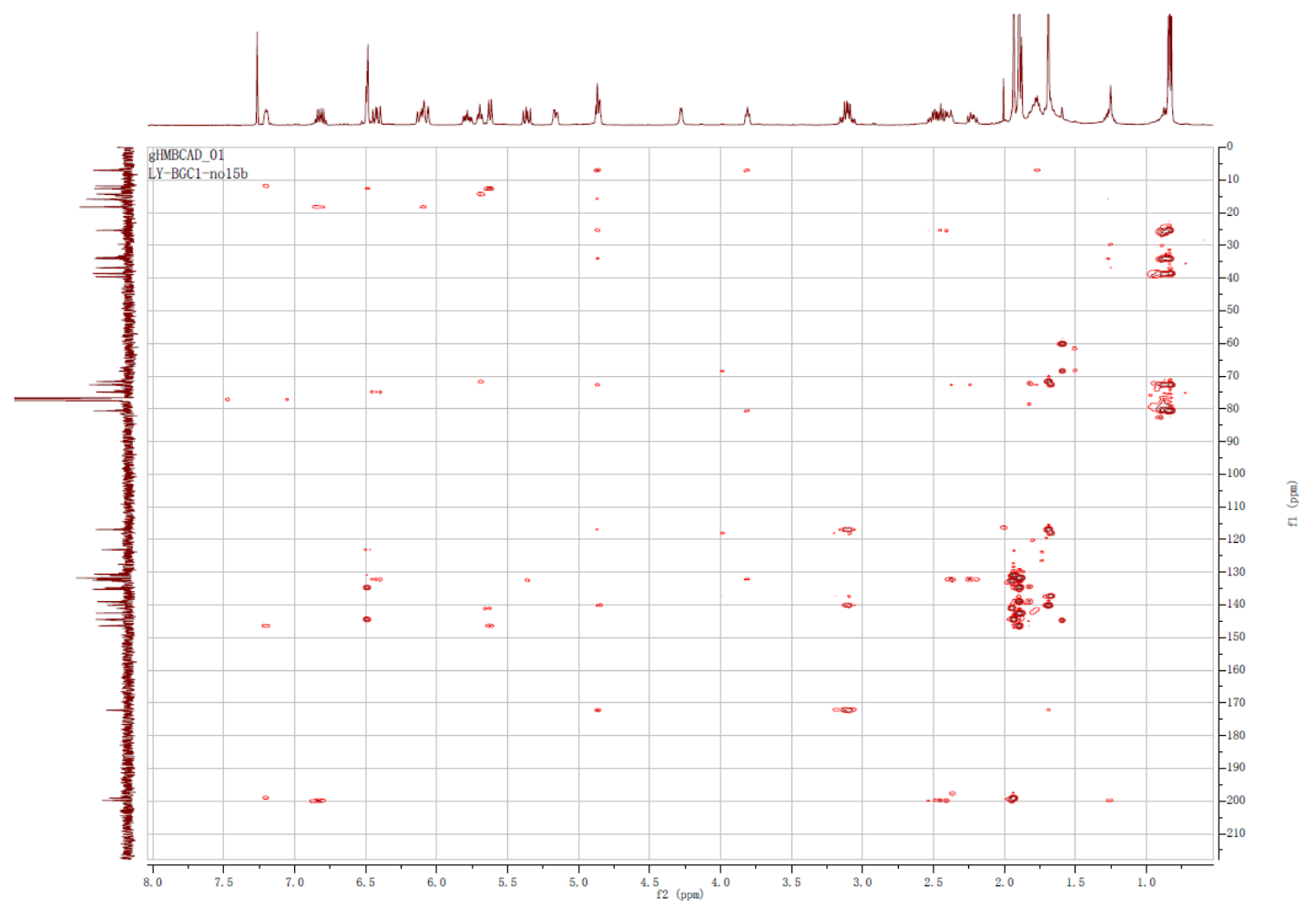

Figure S42. $\mathrm{HMBC}$ spectrum $(125 \mathrm{MHz} / 500 \mathrm{MHz})$ of shuangdaolide $\mathrm{F}(6)$ in $\mathrm{CDCl}_{3}$. 


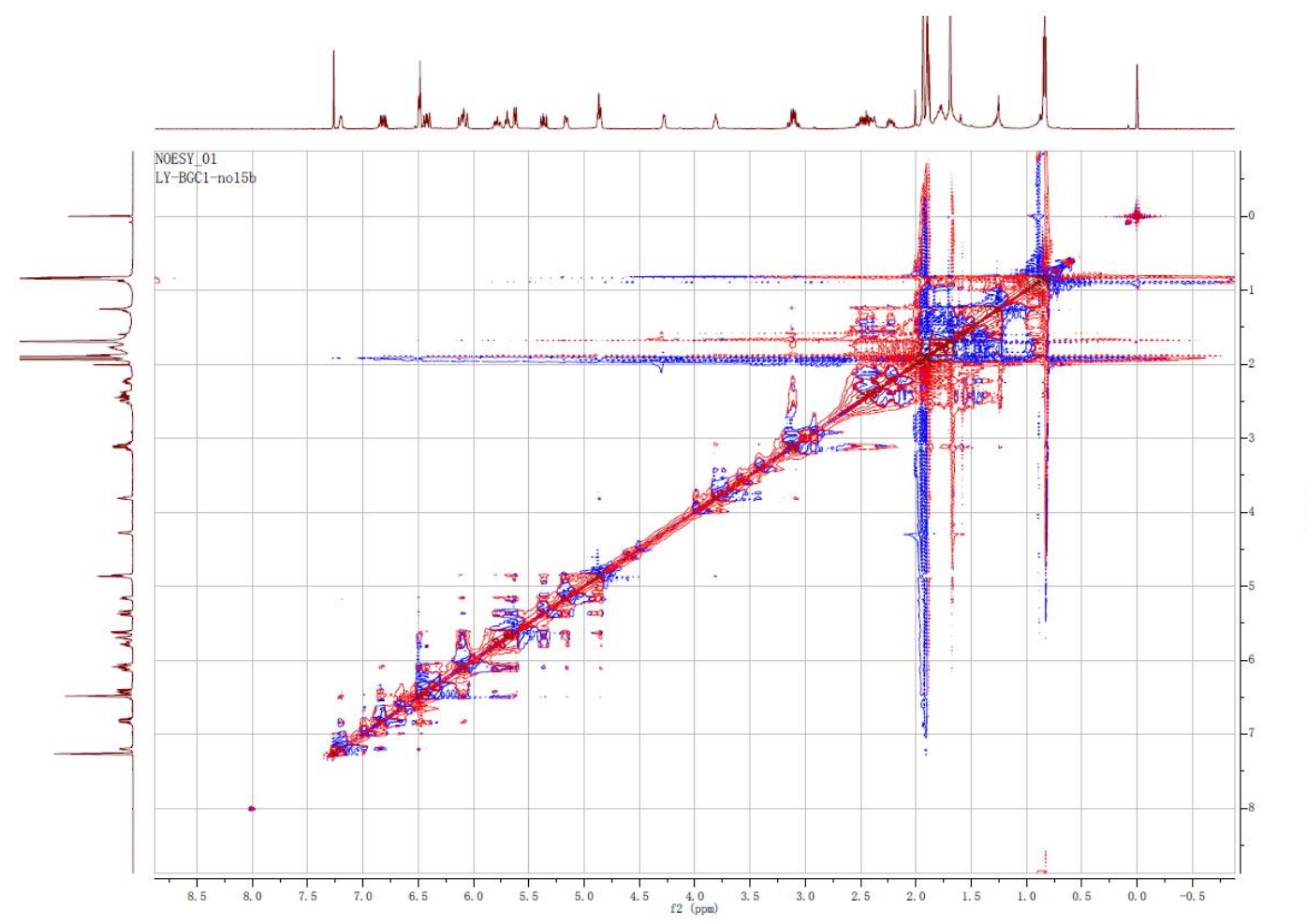

Figure S43. NOESY spectrum (500 MHz) of shuangdaolide $\mathrm{F}(6)$ in $\mathrm{CDCl}_{3}$.

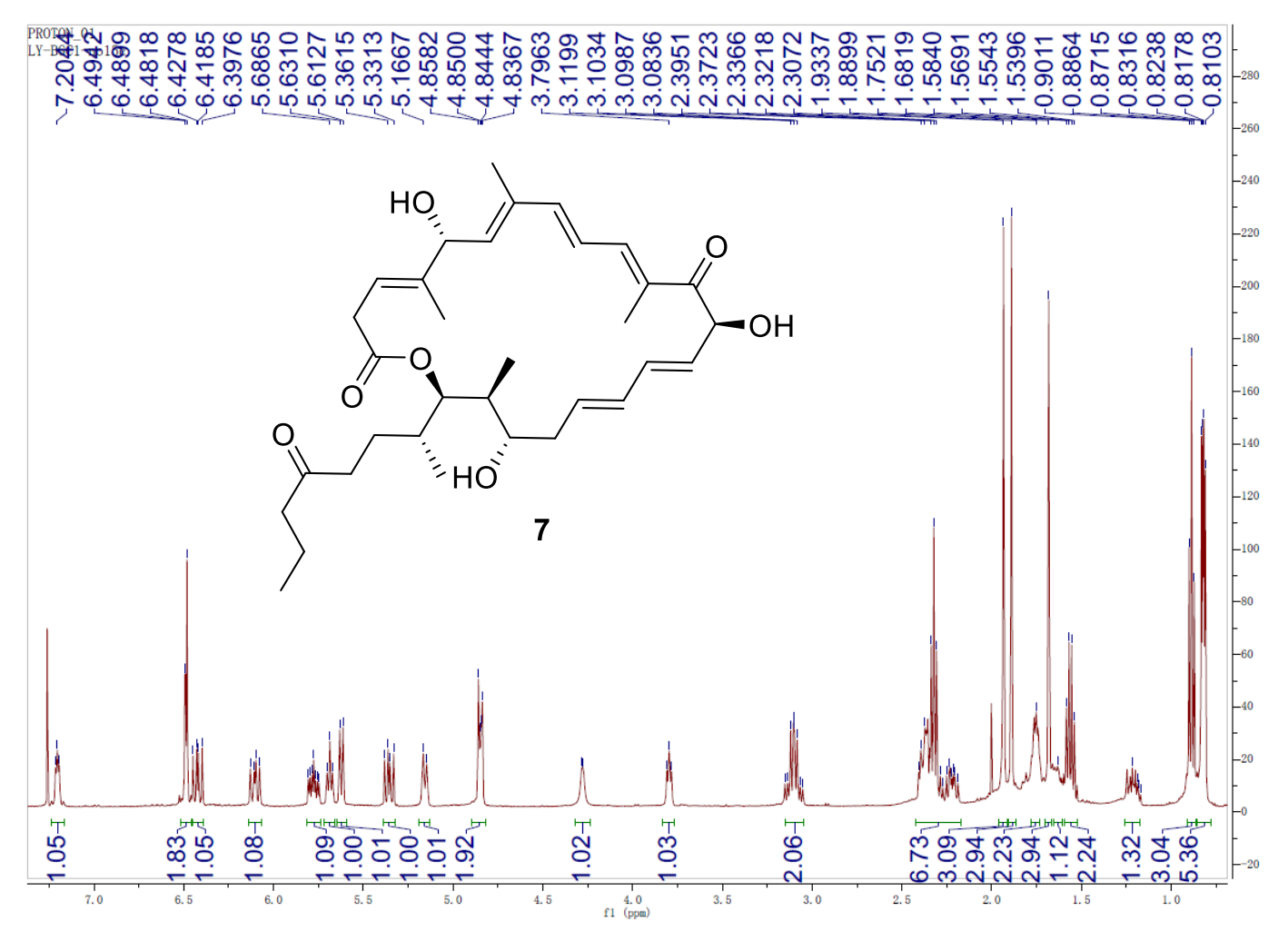

Figure S44. ${ }^{1} \mathrm{H}$ NMR spectrum $(500 \mathrm{MHz})$ of shuangdaolide $\mathrm{G}(7)$ in $\mathrm{CDCl}_{3}$. 


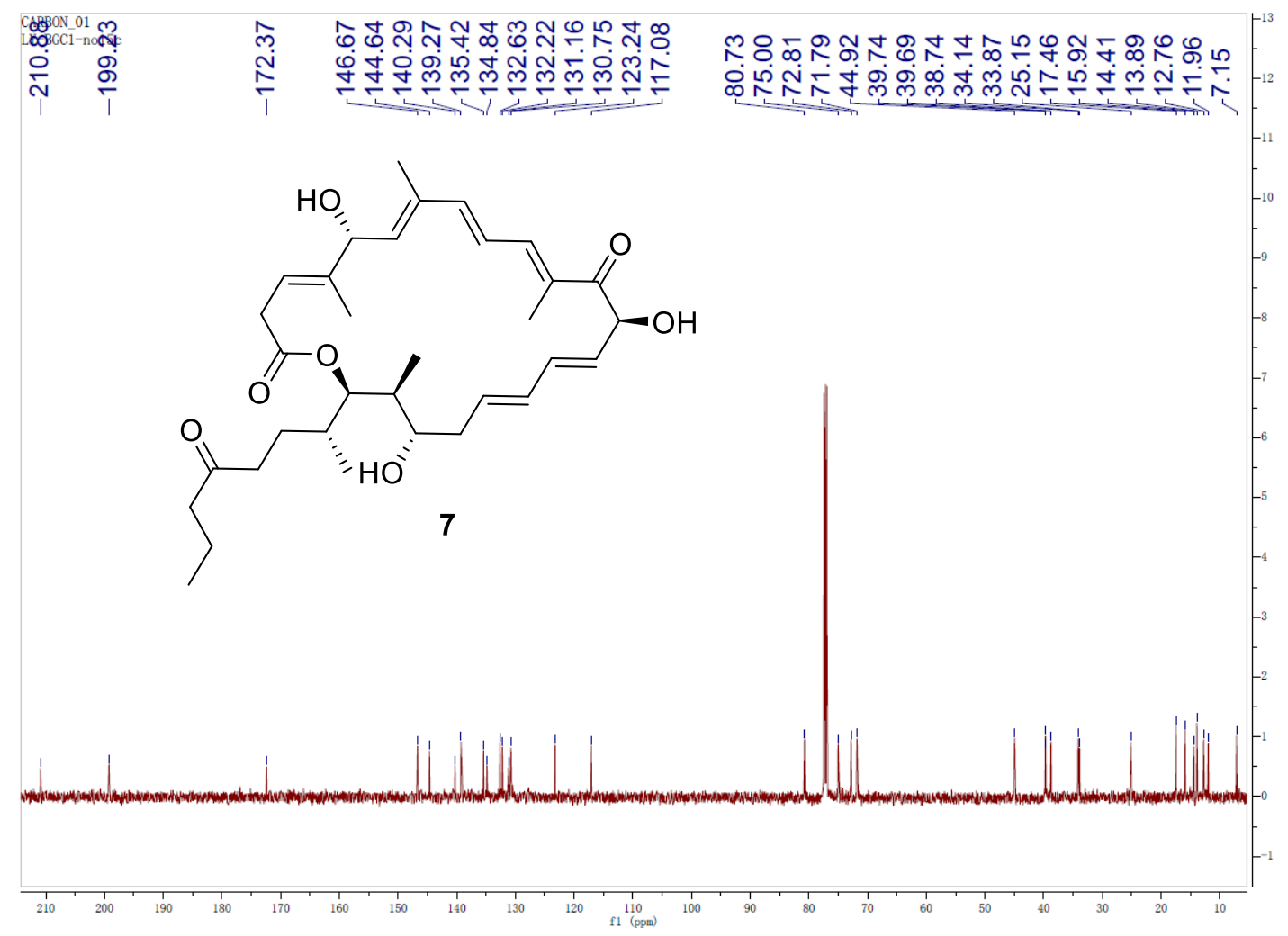

Figure S45. ${ }^{13} \mathrm{C}$ NMR spectrum (125 MHz) of shuangdaolide $\mathrm{G}(7)$ in $\mathrm{CDCl}_{3}$.

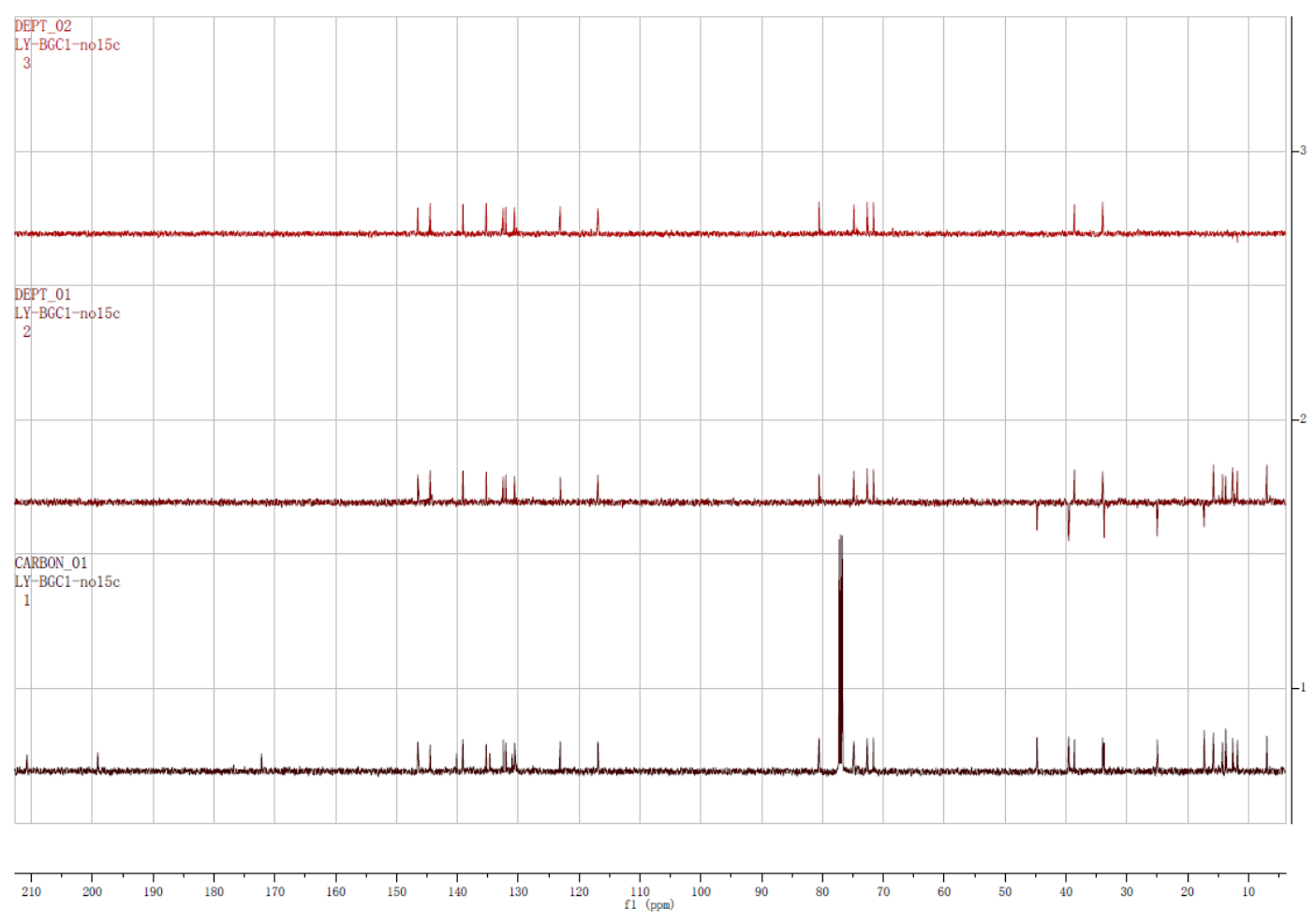

Figure S46. DEPT spectrum (125 MHz) of shuangdaolide $\mathrm{G}$ (7) in $\mathrm{CDCl}_{3}$. 


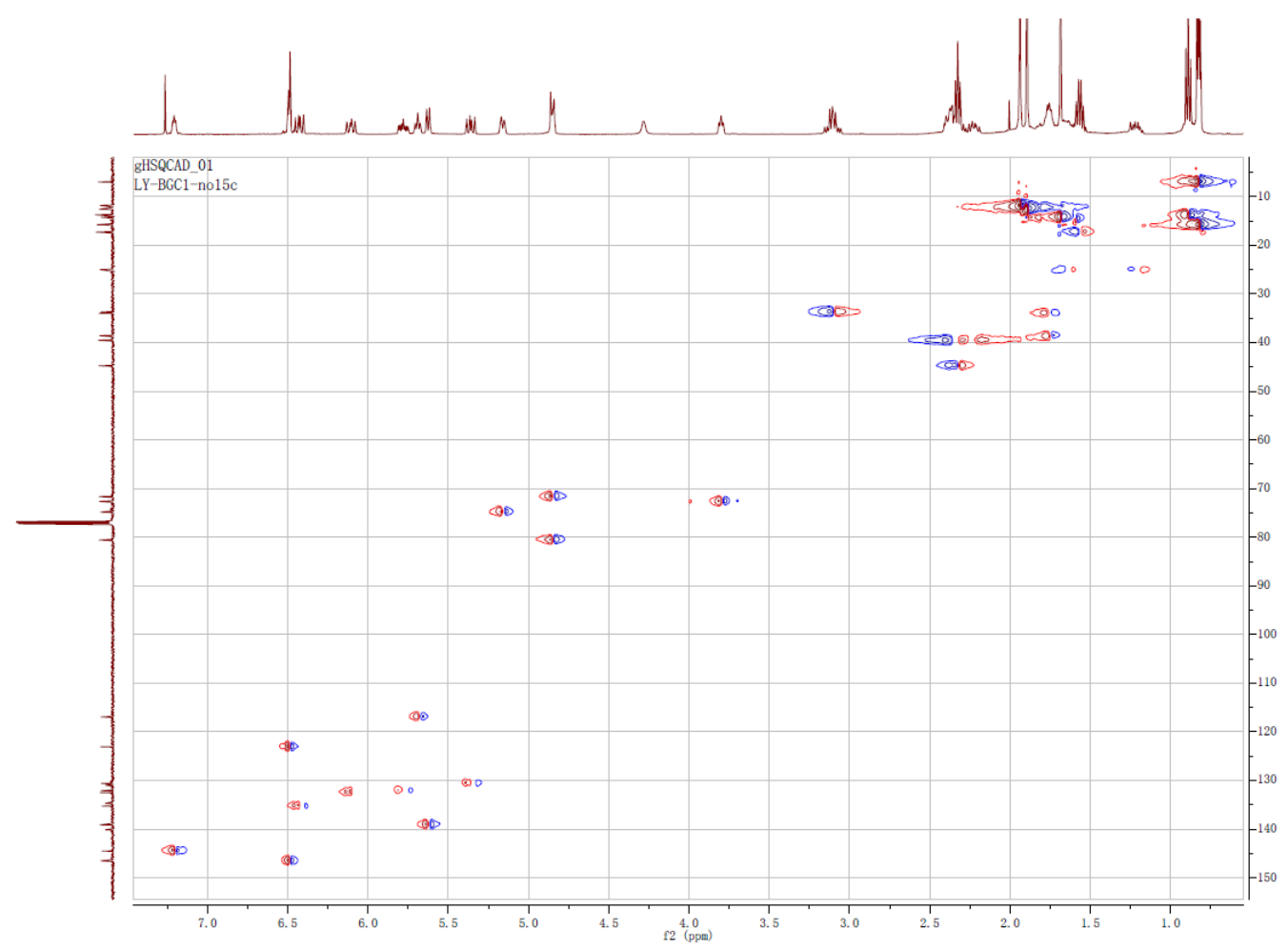

Figure S47. HSQC spectrum (125 MHz/500 MHz) of shuangdaolide G (7) in $\mathrm{CDCl}_{3}$.

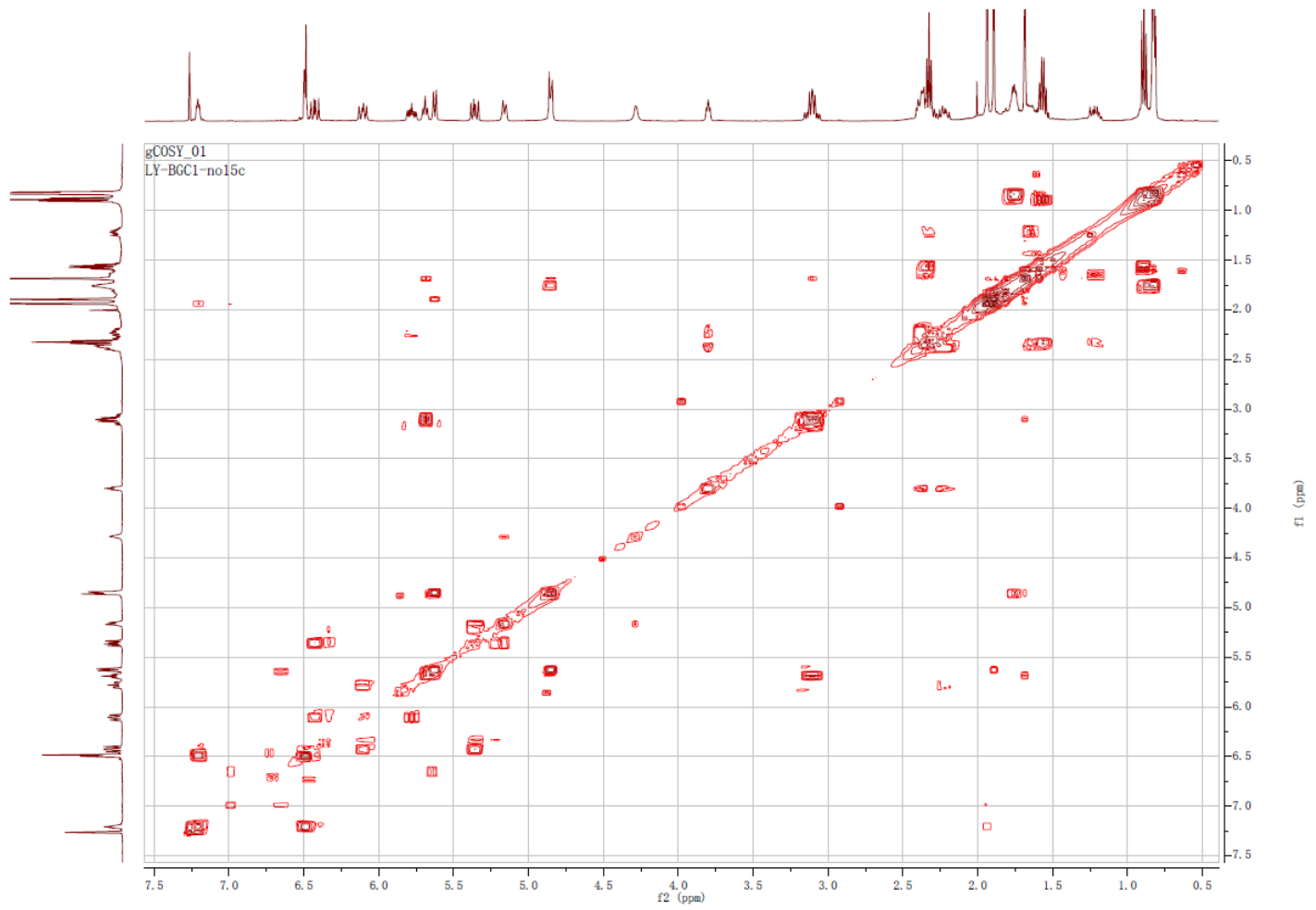

Figure S48. ${ }^{1} \mathrm{H}-{ }^{1} \mathrm{H}$ COSY spectrum $(500 \mathrm{MHz})$ of shuangdaolide $\mathrm{G}(7)$ in $\mathrm{CDCl}_{3}$. 


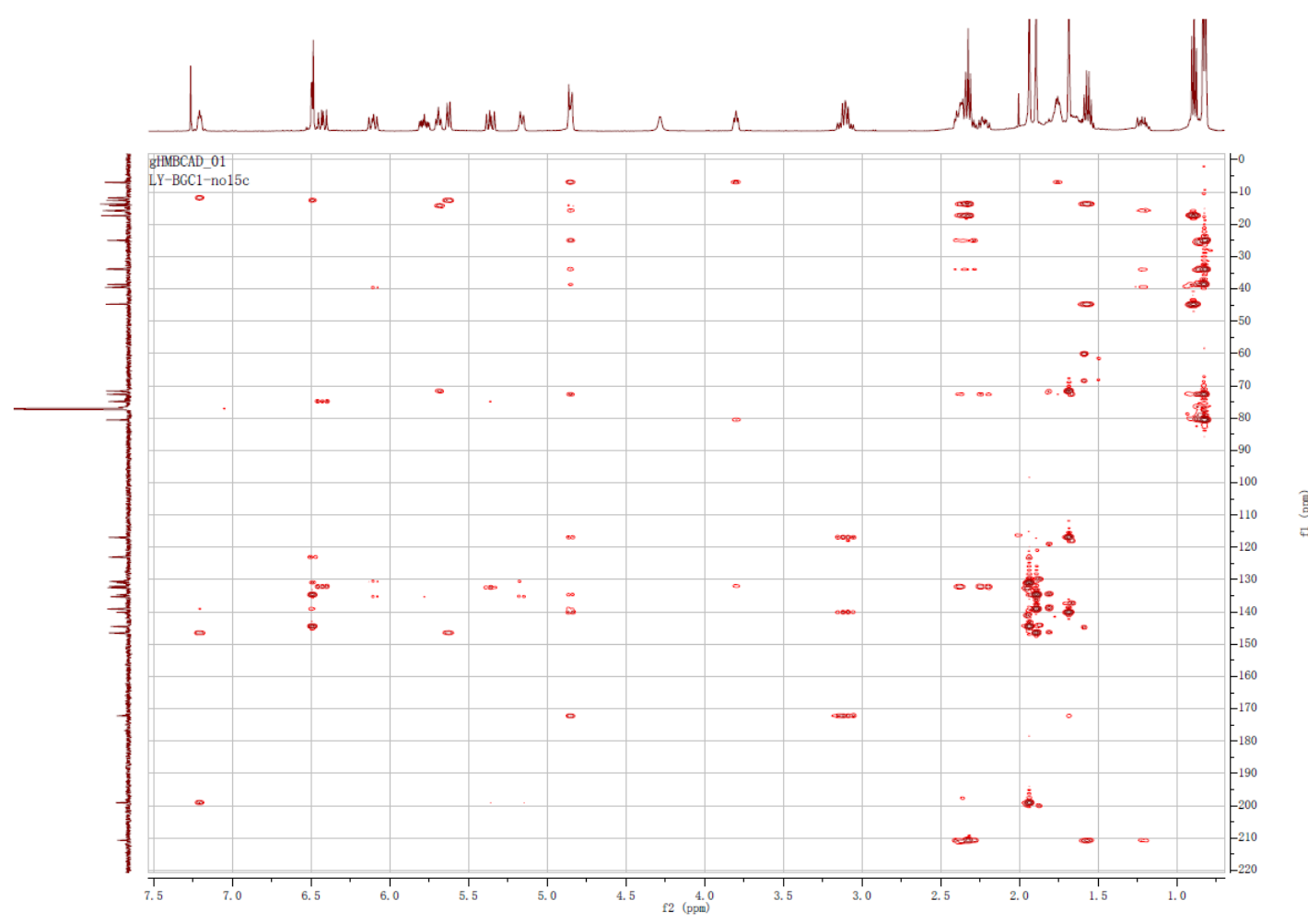

Figure S49. HMBC spectrum (125 MHz/500 MHz) of shuangdaolide $\mathrm{G}$ (7) in $\mathrm{CDCl}_{3}$.

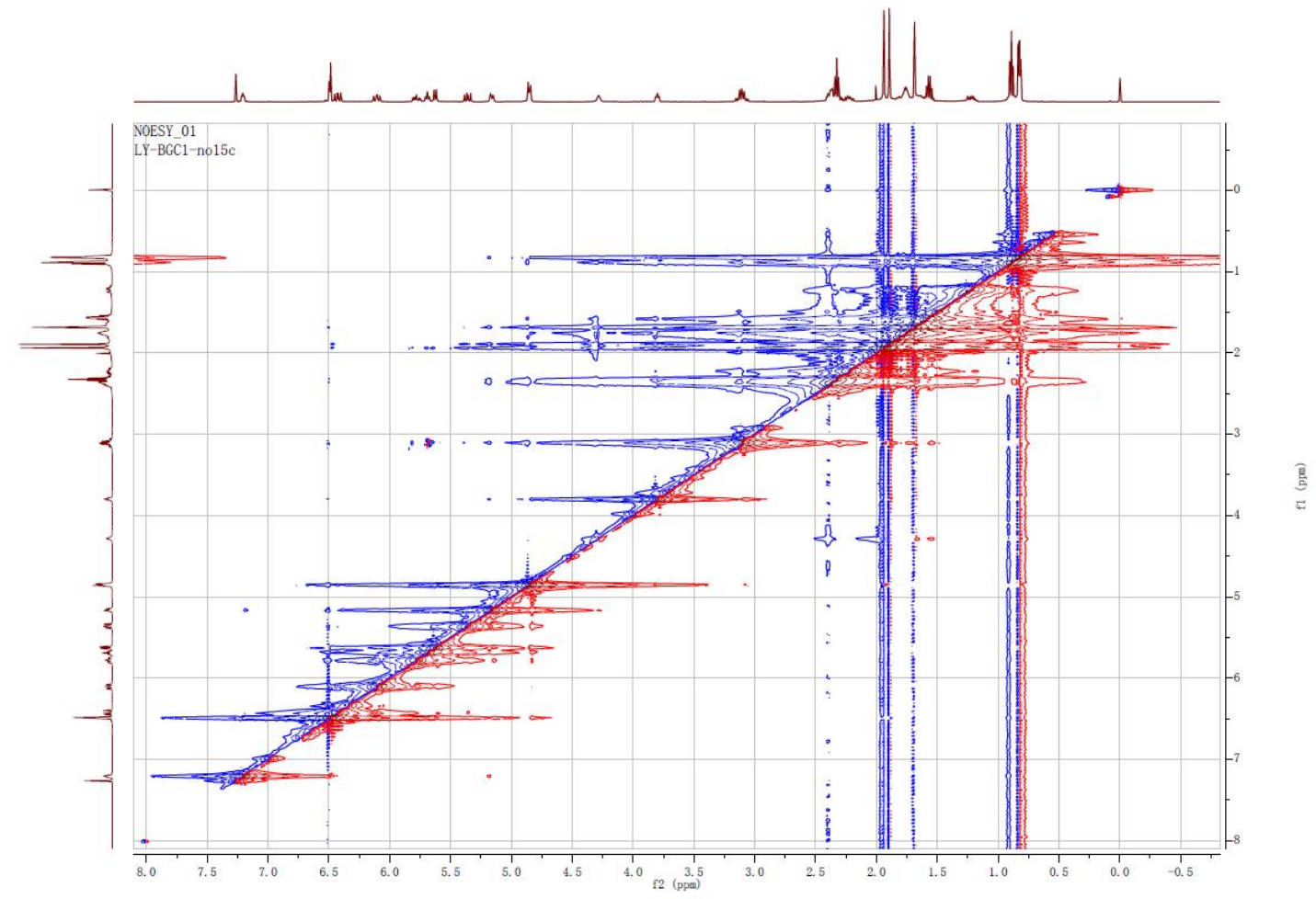

Figure S50. NOESY spectrum (500 MHz) of shuangdaolide $\mathrm{G}(7)$ in $\mathrm{CDCl}_{3}$. 